\title{
THE DESIGN AND FABRICATION OF AUTONOMOUS POLYMER-BASED SURFACE TENSION-CONFINED MICROFLUIDIC PLATFORMS
}

\author{
by \\ MICHAEL J. SWICKRATH \\ Submitted in partial fulfillment of the requirements \\ For the degree of Doctor of Philosophy \\ Dissertation Advisor: Dr. Gary E. Wnek \\ Department of Chemical Engineering \\ CASE WESTERN RESERVE UNIVERSITY
}

January, 2007 


\title{
CASE WESTERN RESERVE UNIVERSITY \\ SCHOOL OF GRADUATE STUDIES
}

\author{
We hereby approve the dissertation of \\ Michael J. Swickrath
}

candidate for the Ph.D. degree *.

(signed)__ Gary E. Wnek

(chair of the committee)

J. Adin Mann, Jr.

Harihara Baskaran

Daniel Lacks

(date) $\quad 01 / 18 / 07$

*We also certify that written approval has been obtained for any proprietary material contained therein. 
Dedicated to the love of my life, Amber Nicole Swickrath, and my loving parents William and Mary Pam Swickrath. 


\section{TABLE OF CONTENTS}

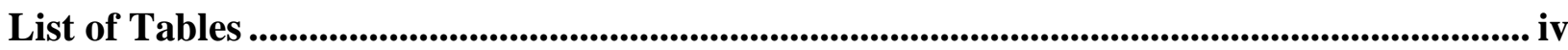

List of Figures......................................................................................................................................................... $\mathrm{v}$

Acknowledgements ....................................................................................................................................... viii

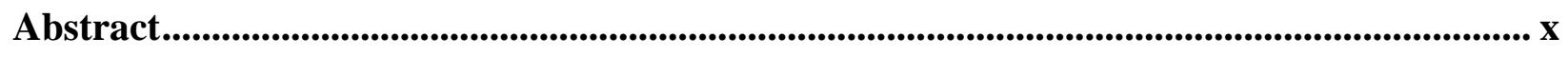

Chapter 1: Introduction to Capillary Driven Microfluidics ................................................................ 1

1.1 Surface Tension-Confined Microfluidics ..................................................................... 1

1.2 Capillary-Driven Microfluidic Physics …………………........................................... 3

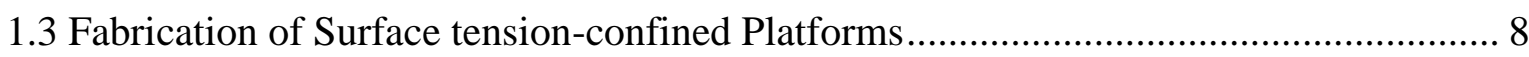

1.3.1 Wetting Mediation through Chemical Patterning .............................................. 8

1.3.2 Curvature Modulation via Topographical Manipulation .................................. 16

1.3.3 Flow Modulation Techniques .................................................................... 19

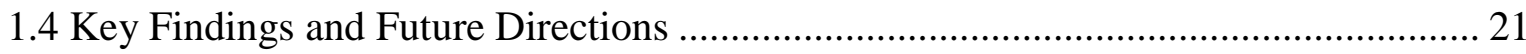

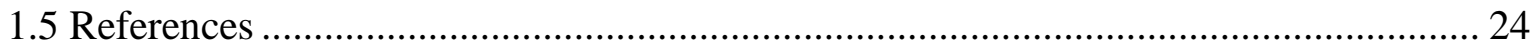

Chapter 2: Fundamentals of Flow in a Microfluidic Regime ..................................................... 27

2.1 Surface Tension and Surface Free Energy ……………………………………..... 27

2.2 Surface Tension Dependency on System Properties ..................................................... 27

2.2.1 Surface tension expansion via contact angle ………………............................. 28

2.2.2 Surface tension dependency on area per volume ……….................................... 30

2.2.3 Thermal influence on surface tension .......................................................... 37

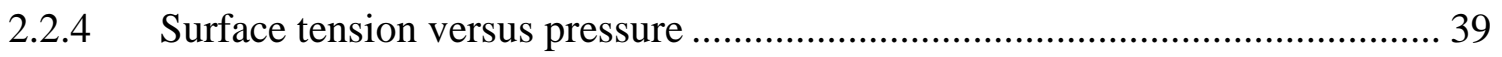


2.3 Young's Equation and Contact angle measurement 42

2.4 Exploitation of Curvature for Capillary Flow ............................................. 46

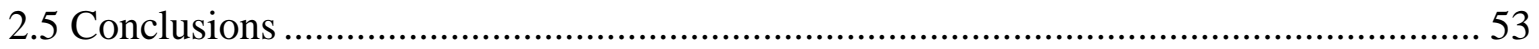

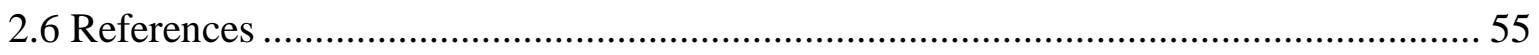

Chapter 3: Design and Fabrication of Autonomous Microfluidic Platforms........................ 59

3.1 Literature Review ................................................................................ 59

3.2 Hydrophilic Patterning of Hydrophobic Substrates ...................................... 62

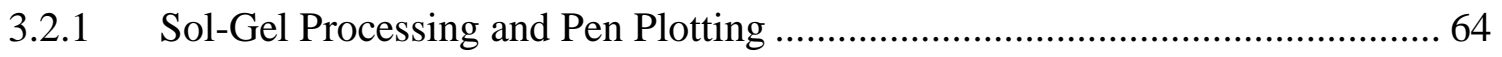

3.2.2 Thick Film Printing of Hydrophilic Polymers .............................................. 68

3.2.3 Thick Film Printing of Three-Dimensional Microfluidic Systems ................... 86

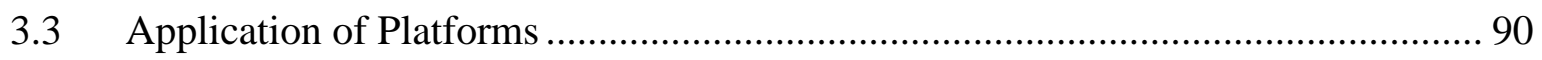

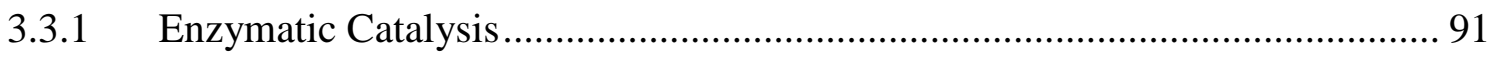

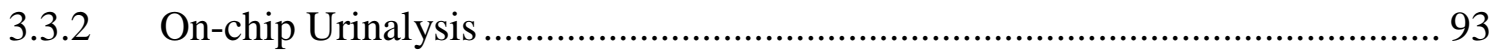

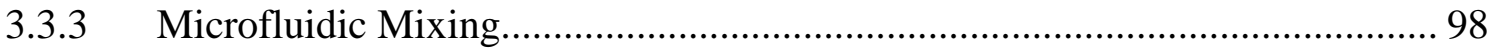

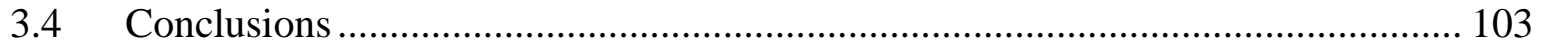

References ....................................................................................... 104

Chapter 4: Alternative Methodology for Platform Fabrication ............................................ 108

4.1 Limitations of current fabrication techniques .................................................. 108

4.2 Thick film printing of materials with intermediate wettability .............................. 108

4.3 Microplasma writing of hydrophilic paths upon polymeric substrates .................... 112 
4.4 Ultraviolet light-mediated path formation. 116

4.4.1 Photoacid generators for side-group hydrolysis .......................................... 116

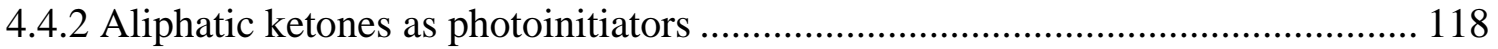

4.5 Concluding Remarks and Suggestions .......................................................... 121

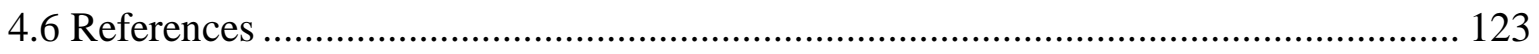

Chapter 5: Future of Surface Tension-Confined Microfluidics .......................................... 125

5.1 Optimizing platforms made via thick film printing .................................... 125

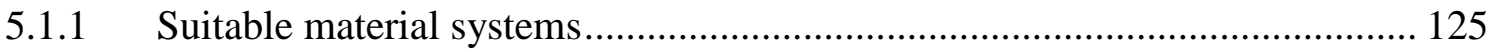

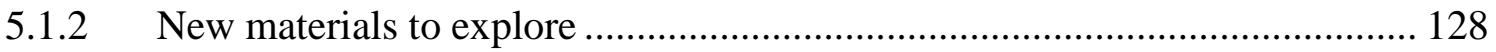

5.1.3 Potential chemical modification of materials ........................................... 129

5.2 Imperative for integration of versatile analyses ........................................ 130

5.2.1 Enzyme-linked immunosorbent assay design ....................................... 130

5.2.2 Fluorescent resonance energy transfer on-chip........................................ 135

5.3 Current Disadvantages of STCM Platforms.................................................... 138

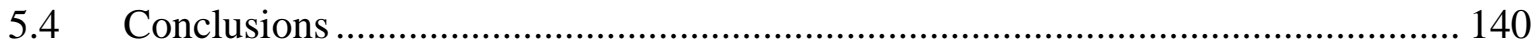

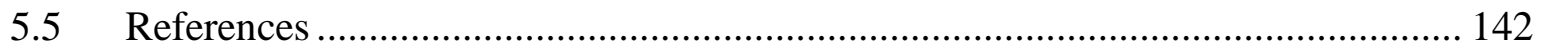

Bibliography ......................................................................................................................................................... 144 


\section{List of Tables}

Table 2.1 Advancing Contact angle values for materials associated with functional STCM platforms as determined via the sessile drop method.

Page 45

Table 3.1 Thick film-printed pattern width as a function of ink concentration and path width.

Page 71

Table 3.2 Summary of calculations and comparison to the Laplace pressure approximation.

Page 85

Table 3.3 Composition of urine analog prepared for on-chip urinalysis (mass calculated for $10 \mathrm{ml}$ of total solution).

Page 95

Table 5.1 Materials investigated for the production of STCM platforms.

Page 126 


\section{List of Figures}

Figure 1.1 Surface-directed microfluidic device fabricated via sol gel processing (A) with a demonstration of the configuration of device and (B) the implementation of the device with colored fluid for visualization (adapted from Lam et al., Langmuir, 2002). (C) Computational fluid dynamic representation of the fluid front of a device fabricated with a patterned bottom substrate and indiscriminately hydrophobic top substrate. (D) The implementation of the device with an unpatterned top substrate (adapted from Bouaidat et al, Lab on a Chip, 2005).

Page 11

Figure 1.2 Geometrical limits facilitating confined capillary transport.

Page 12

Figure 1.3 Demonstration of equilibrated liquid structure based on system geometry and advancing contact angle. Characteristic features include droplet (D), filament (F), pinnedwedges $(\mathrm{pW})$ and corner-wedges $(\mathrm{cW})$. The $+/$ - signs indicate whether the Laplace pressure associated with each morphology is positive or negative, respectively (adapted from Seemann et al., Proc. Nat. Acad. Sci., 2004).

Page 17

Figure 1.4 Illustration of v-groove experiments (A) with the demonstration of system architecture and (B) the experimental platform utilized in the study (adapted Rye et al., Langmuir, 1996).

Page 18

Figure 1.5 (a-e) Thermal induced division of a drop of dodecane on a selectively wetting strip $1000 \mu \mathrm{m}$ wide (elapsed time $=8.5$ seconds). (f-i) Dodecane drop propelled through a $1000 \mu \mathrm{m}$ wide intersection (elapsed time $=104$ seconds). (j-1) Dodecane drop thermally guided across a $90^{\circ}$ angle in a $1000 \mu \mathrm{m}$ wide intersection (elapsed time $=164$ seconds). Adapted from Darhuber et al., 2003.

Page 20

Figure 2.1 Density profile of water versus radial position in a droplet suspended in air.

Page 31

Figure 2.2 Illustration of sessile drop contact angle from (a) isometric view and (b) side view.

Page 42

Figure 2.3 Graphical specification of system boundary conditions for conservative level set calculation of transient capillary transport.

Page 49

Figure 2.4 Conservative level set method results for two-phase capillary dynamics.

Page 50

Figure 3.1 a Configuration of surface-directed microfluidic platform from printed substrate to parallel-plate microfluidic device. b Implementation of surface tension confined microfluidic device.

Page 61

Figure 3.2 Geometry of a high surface tension fluid progressing across a hydrophilic path in a surface-directed microfluidic device. a Isometric view and b side view.

Page 63 
Figure 3.3 Reaction mechanism of sol gel polymerization via the polyalkoxysiloxane route. a Polymerization of polyalkoxysiloxane oligomer to a copolymer glass $\mathbf{b}$ Hydrolysis and syneresis of copolymer glass to $\mathrm{SiO}_{2}$ glass matrix.

Page 66

Figure 3.4 Device fabricated via sol gel processing upon a roughened polystyrene surface.

Page 67

Figure 3.5 Devices made with pen plotting sol gel on (A) polypropylene $\sim 200 \mu 1$, (B) polypropylene $\sim 200 \mu \mathrm{l}$ and $(\mathbf{C}, \mathbf{D})$ polyvinyl chloride $\sim 25 \mu \mathrm{l}$.

Page 67

Figure 3.6 Graphical summary of thick-film printed paths versus polymer concentration.

Page 71

Figure 3.7 Demonstration of autonomous surfaced-directed microfluidic platforms. a Diverging pattern - $19 \mu \mathrm{l}$ b Four-well design - $15 \mu \mathrm{l}$ c 'Microliter' - $15 \mu \mathrm{l}$ d Device implemented with PBS, e SDS, and f DMEM g Device with minimum attainable resolution.

Page 74

Figure 3.8 Scanning electron micrograph indicating relative thickness of a thick film-printed cellulose acetate path encouraged to lift from the substrate material with the tip of a razor blade.

Page 76

Figure 3.9a Capillary dynamics as a function of time and system architecture for a height of 116.4 micrometers and a contact angle of $82.4^{\circ} \pm 1.5^{\circ}$.

Page 79

Figure 3.9b Capillary dynamics as a function of time and system architecture for a height of 229.0 micrometers and a contact angle of $82.4^{\circ} \pm 1.5^{\circ}$.

Page 79

Figure 3.9c Linearized capillary dynamics as a function of time and system architecture for a height of 116.4 micrometers and a contact angle of $82.4^{\circ}$.

Page 80

Figure 3.9c Linearized capillary dynamics as a function of time and system architecture for a height of 229.0 micrometers and a contact angle of $82.4^{\circ}$..

Page 80

Figure 3.10 Reconstructed surfaces utilizing the Monge surface representation. a $w=830.9$ $\mu \mathrm{m}, h=116.4 \mu \mathrm{m} \mathrm{b} w=830.9 \mu \mathrm{m}, h=229.0 \mu \mathrm{m} \mathbf{c} w=1146.9 \mu \mathrm{m}, h=116.4 \mu \mathrm{m} \mathrm{d} w=$ $1146.9 \mu \mathrm{m}, h=229.0 \mu \mathrm{m}$.

Page 84

Figure 3.11 Predicted pressure jump across gas-liquid interface for all configurations investigated. a $w=830.9 \mu \mathrm{m}, h=116.4 \mu \mathrm{m} \mathbf{b} w=830.9 \mu \mathrm{m}, h=229.0 \mu \mathrm{m} \mathbf{c} w=1146.9 \mu \mathrm{m}$, $h=116.4 \mu \mathrm{m} \mathbf{d} w=1146.9 \mu \mathrm{m}, h=229.0 \mu \mathrm{m}$.

Page 85

Figure 3.12 Three-dimensional microfluidic device design capable of facilitating the colorimetric detection of glucose. a Distinguishing ink from substrate and an enhanced view of the tortuous reaction chamber responsible of bringing glucose and reagents into intimate contact. b Annotation of function of designed pattern.

Page 89

Figure 3.13 Demonstration of glucose assay within three-dimensional microfluidic platform.

Page 90 
Figure 3.14 (A) Reaction of substrate with substrate in presence of enzyme to produce yellow product. (B) Demonstration of assay with a droplet of substrate solution upon a 'dot' of encapsulated enzyme. (C) Reaction incorporated with STCM device ( $\sim 50 \mu$ of substrate solution).

Page 92

Figure 3.15 Initial assay results for a $0 \mathrm{mM}$ glucose and $0 \mu \mathrm{M}$ albumin, b $125 \mathrm{mM}$ glucose and $18.3 \mu \mathrm{M}$ albumin, c $250 \mathrm{mM}$ glucose and $37.5 \mu \mathrm{M}$ albumin, $\mathbf{d} 500 \mathrm{mM}$ glucose and 75 $\mu \mathrm{M}$ albumin. Final assay results for e $0 \mathrm{mM}$ glucose and $0 \mu \mathrm{M}$ albumin, f $125 \mathrm{mM}$ glucose and $18.3 \mu \mathrm{M}$ albumin, $\mathbf{g} 250 \mathrm{mM}$ glucose and $37.5 \mu \mathrm{M}$ albumin, $\mathbf{h} 500 \mathrm{mM}$ glucose and 75 $\mu \mathrm{M}$ albumin.

Page 97

Figure 3.16 Chaotic advection in a surface tension-confined microfluidic platform. (a) Demonstration of platform configuration. (b) Control device without checker pattern diffusive mixing only. (c) Device with checker pattern resulting in chaotic advection and homogenization.

Page 100

Figure 3.17 Steady-state microfluidic mixer pattern with fluid pumped through the device. (A) Advancing free surface pre-steady state. (B) Control device without checkerboard pattern. (C) Device with checkerboard pattern. (D,E) Zoomed channel of $(\mathbf{B}, \mathbf{C})$, respectively.

Page 102

Figure 4.1 Formation and derivitization of polyvinyl alcohol.

Page 110

Figure 4.2 Kinetics of hydrolysis of PVB in $1.0 \mathrm{M} \mathrm{HCl}$ through contact angle measurement.

Page 111

Figure 4.3 Demonstration of microplasma setup (adapted from Chiang et al., 2007).

Page 114

Figure 4.4 Contact angle over a period of time for microplasma-patterned PMMA.

Page 115

Figure 4.5 Schematic of hydrolysis of an acrylic polymer enabling wettability mediation.

Page 117

Figure 4.6 Proposed mechanism for the (a) photodecomposition of 2-butanone and (b) subsequent self-initiation of acrylic acid grafting to HDPE surface.

Page 120

Figure 5.1 Side view of preliminary design of multi-tiered surface tension-confined microfluidic platform facilitating the ELISA technique in an on-chip format.

Page 134

Figure 5.2 Demonstration of molecular beacon systems. (a) Hybridization results in fluorescent signal gain (Kim et al., 2007). (b) Hybridization results in signal loss (Jung et al., 2007).

Page 136

Figure 5.3 Mixer design patterns for $6 \mathrm{~cm}$ long paths. (a) Control pattern, (b) checker board (see section 3.3.3) (c) staggered herringbone adapted from Stroock, et al., and (a) Weierstrass herringbone pattern adapted from Camesasca, et al.

Page 138 


\section{Acknowledgements}

As I began my research at Case Western Reserve University a few years ago, the concept of completing substantial research warranting the compilation of a dissertation seemed quite daunting. As time passed and I developed new perspectives and proficiencies, I realized that the journey was much more important than the destination. Alas, the destination seems to be approaching in time to begin future journeys. The following work would never have materialized had it not been for the support a multitude of colleagues, friends and family members.

First and foremost, I sincerely thank my advisor, Professor Gary E. Wnek, for his mentorship and fostering of my technical development. Prof. Wnek encouraged an out of the box approach to innovation that I look forward to taking into future projects. Lastly, Prof. Wnek supported the exposure to managerial aspects and the appreciation of how to integrate technical aspects of a project together with project management, accounting \& finance, commercialization and marketing during my tenure at Case. Consequently, I have learned invaluable lessons that transcend the scope of most Doctorate of Philosophy programs and I am greatly indebted for this.

In addition, I thank my thesis committee for technical guidance on many aspects of my research. Specifically, Prof. J. A. Mann Jr. for assistance with interfacial phenomena, Prof. H. Baskaran for aid with tissue culture and biomolecular processing and Prof. D. Lacks for advice on numerical analysis. I also thank the Case Western Reserve University, Department of Chemical Engineering, for confidence in my abilities, financial support and facilities to practice research. I also thank the Infoscitex, Corporation (Waltham, MA) for financial support and encouragement in the early stages of device design and 
implementation. As well, I thank Prof. I. Manas-Zloczower and Dr. Marco Camesasca for many enlightening discussions and assistance with CFD and computational mixing investigations.

I thank the Wnek research group - especially Meg Smith for discussions on science and technology, as well as being a friend. Furthermore, I have had the pleasure to work with numerous talented undergraduate/graduate student researchers who have helped me with data collection and protocol optimization while developing my capacity to mentor. I thank Trevor Ilse for aid with platform development, Sujai Shriram and Patrick Gary for investigating commercialization aspects related to the project, Beth Samuelson for assistance with tissue culture and Steven Burns for support with a wide variety of projects. I express gratitude Jason Mazzoccoli and Ram Subbaraman for support and friendship in general throughout this process. I thank Laurie Dudik for advice troubleshooting microfabrication techniques.

In addition, I thank all of the rest of the graduate students in which I have had the opportunity to study, relax, play intramural sports, work on committees or spend time with in general. To write all of the names would probably dwarf the rest of the work to be included within this manuscript.

Finally, I am forever grateful for my family and loving wife because without there support and encouragement, I would not have had the perseverance to accomplish this feat. Thank you so much for motivating me or even giving me the shoulder to cry on when the stress seemed too much. Thank you all.

-Michael J. Swickrath

Cleveland, $\mathrm{OH}$

November 2007 
The Design and Fabrication of Autonomous Polymer-Based

Surface Tension-Confined Microfluidic Platforms

\section{Abstract}

by

\section{MICHAEL J. SWICKRATH}

The field of microfluidics, lab-on-a-chip technologies in particular, promises the capacity to automate sophisticated laboratory analyses into a platform that can be implemented by a user with minimal analytical experience. However, the fabrication methods traditionally employed to manufacture microfluidic devices are cost ineffective and time intensive. Consequently, current production techniques render exploiting this technology for clinical application problematic. This work describes an alternative fabrication technique to mitigate the aforementioned problems through surface tensiondriven flow. Hydrophilic conduits are patterned on a variety of commodity polymeric substrates. The opposing two-dimensionally patterned devices are brought within close proximity for the fabrication of a parallel plate configured microfluidic device. The microfluidic platforms demonstrate the ability to facilitate spontaneous capillary pumping with a high degree of precision and minimal expenditure of fluid reagent.

In particular, several cost-effective fabrication procedures are illustrated as well as the capacity to manipulate fluids within the platforms utilizing volumes less than 20 total microliters. Furthermore, applications are demonstrated within the devices such as enzymatic-catalysis, on-chip urinalysis (i.e. glucose and protein detection), and micromixing; 
demonstrating the efficacy of the platform to automate fluid transport concomitantly with reaction processes. In addition, preliminary designs and protocols are suggested in the last chapter of this work for surface tension-confined devices capable of performing enzymelinked immunosorbent assay (ELISA) and fluorescence resonance energy transfer (FRET).

Moreover, theoretical aspects of microfluidic flow are explored within this document including the physics of wetting and wetting energetics, factors influencing surface tension (and thereby the system driving force), the conservative level set method coupling two-phase flow and the Navier-Stokes equations, the expected velocity and Reynolds number of the advancing fluid front within the microfluidic platforms, calculating the curvature-based pressure jump across the advancing meniscus and non-dimensional numbers pertinent to capillary-driven microfluidic flow.

The extension of these results suggests that autonomous micro-analytical systems may be fabricated with limited rigor and mechanical components. Consequently, commercialization opportunities for this novel microfluidic platform are under consideration. 


\section{Chapter 1: Introduction to Capillary Driven Microfluidics}

\subsection{Surface Tension-Confined Microfluidics}

The field of microfluidics promises the capacity to automate sophisticated laboratory analyses into a diminutive platform that can be implemented by a user with minimal analytical experience negating the necessity for trained personnel and/or specialized equipment. Often an analogy is drawn towards the integrated circuit revolution which facilitated the automation of large-scale computation. Similarly, a great deal of optimism exists that microfluidics may have such an impact on chemistry, biology and medicine, resulting in a drastic decrease in the amount of expensive reagent or invasive fluid samples required while enabling higher degrees of throughput and parallelization.

Such microfluidic devices have been successfully developed. Researchers have effectively integrated an enzyme-linked immunosorbent assay into an on-chip platform (Sato et al., 2004) as well as developed chips for DNA analysis (Burns, 1998) and novel applications of microfluidics towards fuel cell development (Choban et al., 2004), among a plethora of other innovative utilizations. However, the fabrication methods traditionally employed to manufacture microfluidic devices tend to be cost ineffective and time intensive. In addition, fabrication techniques may require clean-room facilities or batch processing further complicating production scale-up. Consequently, current production techniques render exploiting this technology problematic for wide-scale deployment. This manuscript describes alternative fabrication techniques to mitigate the aforementioned problems. Specifically, spontaneous surface tension-confined capillary pumping of fluids with 
appropriate surface tension and viscosity is enabled by patterning energetic discontinuities or topographical features upon a surface.

However, before surface tension-confined microfluidics can be employed towards solving problems in chemistry, biology and medicine, a thorough understanding of the underlying physics must be established. This task becomes non-trivial as fluidic miniaturization results in dramatic changes to the fundamental physics manifested at small length scales (Squires \& Quake, 2005). For example, mass transport within microfluidic devices is a challenge as viscous dissipation dominates at small length scales rather than inertial effects. This implication suggests microfluidic flow is nearly devoid of convection enabling the elimination of non-linear terms in the Navier-Stokes equations for incompressible flow. Although the lack of inertia at small length scales seems to indicate the analysis of microfluidic fluid transport is straightforward, quite the opposite is true. Other non-linear phenomena that may not be as familiar at the macro scale become apparent as surface area to volume ratios increase. In particular, surface curvature and three-phase boundaries must be taken into account.

With regard to surface tension-confined microfluidics, patterning discontinuities in surface energetics results in deformation of the solid-liquid contact line (Darhuber et al., 2006). Such deformations can either promote or impede capillary break-up and formation of 'bulges'. Consequently, discontinuous energetics serves as a mechanism to harness for autonomous fluid transport. Furthermore, other implications arise as a result of the capacity to manipulate interfacial curvature at the microscale. As the surface area to volume ratio experienced within microfluidic devices is rather high, the overall shape and stability of boundaries and interfaces can be dramatically affected by minute amounts of surface or fluid 
contaminants. Accordingly, surface tension-confined microfluidic devices warrant proper surface cleaning protocols, storage procedures and reagent preparation to ensure reproducible and reliable device function. Moreover, since reagents may necessarily contain surface-active components, adsorption dynamics may be an important factor in capillary pumping.

The breadth of this chapter seeks to address a review of fabrication techniques, characterization and analysis of microfluidic devices implementing the surface tensionconfined approach. Furthermore, key research findings will be explicated along with aspects of this approach requiring future investigation. As a result, the methodology to implement the surface tension-confined approach will be established along with a suitable record of pertinent literature regarding this innovative technique. However, before doing so, a brief diversion into the treatment of fluid physics for systems exhibiting high surface area to volume ratios may lend much insight in developing a robust understanding and appreciation of capillary-driven microfluidic flow.

\subsection{Capillary-Driven Microfluidic Physics}

As mentioned earlier, microfluidic devices in general can be characterized as lacking significant inertia. Consequently, the non-linear terms rendering solutions to the NavierStokes equations rigorous, can be neglected in analyzing most microfluidic systems. However, the Navier-Stokes approach is not necessarily an optimal strategy towards analyzing microfluidic systems as boundary conditions tend to be very difficult to define as slip velocities need to be considered. Furthermore, the slip velocities are specific to length scale and are generally not well known or understood (Wilkes, 2006). Consequently, empirical approximations and scaling arguments are commonly used in the characterization 
of microfluidics physics, especially when a free surface is involved as in the case of capillarity.

One common approach to treating capillary microfluidic phenomena entails equating the fluid volumetric flowrate to a ratio of pressure drop, $\Delta P$, per resistance, $R$, in a fluid channel as developed by Washburn circa 1921 (Washburn, 1921). Here, $A_{c s}$ represents crosssectional area of a fluid filament (assumed to be constant) and $x$ represents the timedependent location of the meniscus position.

$$
A_{c s} \frac{d x}{d t}=\frac{\Delta P}{R}
$$

Considering capillary dynamics, the pressure drop term is often described by the Laplace equation, $\Delta P=2 \gamma H$, where $\gamma$ represents liquid surface tension and $H$ represents the mean curvature of the liquid-gas interface. Often two distinct radii of curvature are invoked to employ the Laplace equation in the following form (Adamson \& Gast, 1997).

$$
\Delta P_{c}=\gamma\left(\frac{1}{r_{1}}+\frac{1}{r_{2}}\right)
$$

Curvature is usually assumed to follow geometries that can be fit to a circle (or sphere in the three-dimensional case) in the Laplace equation; this is not typically the case but usually results in a decent approximation (Henriksson \& Eriksson, 2004). Further, for straight paths, $r_{2}$ can be considered as infinite and approximations can be made for $r_{1}$ (Zhao et al., 2001). In Eq.(1.3), $\theta_{I}$ indicates the fluid-solid contact angle at the three-phase interface. 


$$
r_{1}=\frac{h}{2 \cos \theta_{I}}
$$

Moreover, resistance terms exist for common geometries such as rectangular ducts (Squires \& Quake, 2005).

$$
R_{D}=\frac{12 \mu x}{w h^{3}}\left\{1-\frac{h}{w}\left[\frac{192}{\pi^{5}} \sum_{n=0,1,2 \ldots}^{\infty} \frac{1}{(2 n+1)^{5}} \tanh \left(\frac{(2 n+1) \pi w}{2 h}\right)\right]\right\}^{-1}
$$

Here $w$ represents channel width, $h$ represents channel height and $\mu$ represents the viscosity of the fluid. In the case where $w>>h$, the bracketed term can be neglected while maintaining sufficient accuracy through the approximation.

By substituting Eq.(1.3) into Eq.(1.2) and subsequently utilizing modified Eq.( 1.2) and Eq.( 1.4) with the general Washburn equation, one finds that capillary dynamics tend to follow Eq.( 1.5) with a general geometry-dependent characteristic length scale, $L$ (Squires \& Quake, 2005). For cylindrical capillaries, $L$ can be represented as radius. For other geometries, one might choose to represent $L$ by the hydraulic diameter, $D_{h}$ (i.e. $D_{h}=$ $4 A_{c s} / P, P$ represents wetted perimeter of the fluidic channel).

$$
x \sim\left(L \frac{\gamma}{\mu} \cos \theta t\right)^{1 / 2}
$$

Consequently, the time-dependent fluid front position in a surface tension-confined microfluidic device tends to be dependent on system geometry, intrinsic fluid properties, fluid-substrate interactions and the square root of time. This dependency has been derived theoretically and observed for cylindrical 'caps' on chemically patterned surfaces as well as for v-groove geometry (Darhuber et al., 2006, Mann Jr et al., 1995, Romero \& Yost, 1996, 
Rye, Mann et al., 1996, Rye, Yost et al., 1996). Noting that the ratio $\gamma / \mu$ is a characteristic capillary velocity, $U_{c}$, enables the rearrangement $x \sim\left(L U_{c} \cos \theta t\right)^{1 / 2}$. This relation perhaps leads to a more tangible description of fluid specific capillary transport.

Outside of the capillary dynamics approach, scaling arguments and dimensionless analysis can provide valuable insight into fluid physics on the micro/nano-liter scale. An exhaustive review of these approaches has been conducted for a variety of microfluidic techniques (Squires \& Quake, 2005). However, for this article, we will strictly consider scaling metrics and dimensionless numbers pertinent to the surface tension-confined microfluidic approach.

First, we will consider perhaps the most often applied dimensionless number toward fluid transport, the Reynolds number. The Reynolds number is a measure of inertial to viscous forces and is dependent upon fluid density $\rho$, and viscosity $\mu$, as well as characteristic velocity $U$ and the characteristic length scale $L$ which can be written as a ratio of velocities.

$$
\operatorname{Re} \equiv \frac{U}{\mu /(\rho L)}
$$

As mentioned earlier, the lack of convection in microfluidic devices indicates inertial effects are minimal. Consequently, viscous forces dominate. Reynolds numbers characteristic of microfluidic devices are generally on the order of $\mathcal{O}\left(10^{-6}\right)$ to $\mathcal{O}(10)$ (4). Furthermore, the transient time required to achieve this laminar flow goes according to $\tau \sim \rho L^{2} / \mu$. Consequently, one can see that flow in microfluidic devices tends to be rather devoid of turbulence. 
Another relevant number in the analysis of microfluidic devices in general is the Péclet number; which yields the ratio of convective transport to diffusive transport where $D$ represents the diffusion constant of a molecule within a particular fluid.

$$
\mathrm{Pe} \equiv \frac{U L}{D}
$$

As already indicated, flow is quite laminar in microfluidic devices which results in great difficulty in attaining adequate mixing for chemical reaction processes. Scales for the general time $\left(\tau \sim w^{2} / D\right)$ and length $\left(z \sim U w^{2} / D\right)$ to achieve mixing in a low Péclet number devices are known considering a channel of width $w$. One example of the application of these scales for a novel purpose is the development of liquid-liquid extraction processes in an on-chip format (Kamholz et al., 2001).

Surface forces tend to be inherently important for capillary-driven microfluidic devices as surface area to volume ratios are rather high and surface tension-induced interfacial curvature is significant enough to promote capillary wicking. An additional dimensionless number particularly important to surface tension-confined microfluidics is the capillary number utilized to measure viscous to interfacial forces.

$$
\mathrm{Ca} \equiv \frac{U}{\gamma / \mu}
$$

For chemically patterned devices depending on hydrophobic/hydrophilic confinement in particular, the capillary number must be sufficiently low (i.e. $\mathrm{Ca}<<1$ ) in order to maintain fluid confinement within intricate geometry implying $\Delta P \leq \gamma / w$ (Squires \& Quake, 2005). 
The above analyses lend insight into the behavior of fluids within surface tensionconfined microfluidic devices. Now that an intuitive framework of the fluid physics within microfluidic platforms has been established, the contemplation of successful platform fabrication techniques and their associated applications is worthy of consideration.

\subsection{Fabrication of Surface tension-confined Platforms}

Several approaches exist to manufacture surface tension-confined microfluidic platforms. Examples of relevance to this article include the chemical patterning of regions exhibiting drastic and abrupt disparity in surface energies and the manipulation of surface topography to influence mean curvature. Furthermore, some alternative techniques will be discussed as potential applications to surface tension-confined microfluidics such as thermal actuation and passive gate fabrication with coatings that enable contact angle mediation via photochemical reactions or the presence of water.

\subsubsection{Wetting Mediation through Chemical Patterning}

One of the first examples of a microfluidic device fabricated through chemical patterning involves the deposition of hydrophobic 'curbs' upon an otherwise hydrophilic glass substrate for the purpose of applications such as capillary electrophoresis (Oh, 1999). However, a great deal of investigation has occurred since these initial studies were conducted.

Initial investigations focused on the relationship between fluid morphology and surface energy. Gau et al. investigated the shape instability that liquids undergo when an appropriate amount of liquid is adsorbed on a hydrophobic surface patterned with hydrophilic 
microstripes (Gau et al., 1998). Hydrophilic stripes were deposited via thermal vapor deposition of $\mathrm{MgF}_{2}$ (stripe thickness $\sim 20 \mathrm{~nm}$ ) upon hydrophobic silicone rubber or thiolated gold substrates. The patterned surface was then exposed to sufficiently humid air and cooled to force the condensation of liquid upon the surface. The results indicate that initially, water adsorbs forming a structure with a cylindrical cross-section; however, when an adequate amount of water is adsorbed, instability can occur resulting in bulge formation. This instability is quite different from the classical Rayleigh instability; rather it is a bifurcation in two different morphologies of constant mean curvature (Gau et al., 1998). A linear stability analysis indicates that the cylinder is locally stable so long as the wavelength of the shape deformation $\lambda$, exceeds a critical value $\lambda_{c}$, based upon the hydrophilic path width $a$ and advancing contact angle $\theta$.

$$
\lambda_{c}=a \frac{\theta}{\sin \theta}\left[\frac{\pi / 2}{\theta^{2}-(\pi / 2)^{2}}\right]^{1 / 2}
$$

Consequently, Gau suggests that it is in fact feasible to build two-dimensional microfluidic devices through chemical patterning techniques.

These results were also independently confirmed by other groups (Darhuber et al., 2006, Darhuber et al., 2001). Darhuber et al. developed disparities in hydrophilicity on a [001]-oriented p-type doped silicon wafers coated with a self-assembled monolayer of octadecyl-trichlorosilane (OTS). In all of the studies (Darhuber et al., 2006, Darhuber et al., 2001, Gau et al., 1998), the experimental results were found to be in good agreement with theoretically determined surface morphology through the SURFACE EVOLVER software package. The SURFACE EVOLVER software package is an interactive tool to study the 
equilibrium surface shapes of liquids subject to surface tensions and other energies under various constraints. The SURFACE EVOLVER evolves the surface toward minimal energy by a gradient descent method (Brakke, 1992). The SURFACE EVOLVER software package is a powerful tool for the theoretical investigation of surface tension-confined microfluidics and the equilibrated surface shapes within such devices. Most often, gravity can be neglected in the SURFACE EVOLVER analysis as indicated by the low Bond number experienced in microfluidic devices (i.e. Bo $<<1$ ). Specifically, the Bond number is a measure of body forces (i.e. gravity) per surface forces (i.e. capillarity) where $\Delta \rho$ indicates the change in density across a surface, $g$ is the gravitational constant, $L$ is a characteristic length of the capillary cross-section and $\gamma$ is the surface tension of the fluid.

$$
\text { Bo } \equiv \frac{\Delta \rho g L^{2}}{\gamma}
$$

A low Bond number indicates that capillary forces dominate over gravitational effects in surface tension-confined microfluidic platforms (Darhuber et al., 2006).

These findings were later extended towards the development of autonomous microfluidic platforms employing surface tension-confined fluid transport. Several studies indicate that microfluidic platforms can be manufactured by chemically patterning substrates in combination with correspondingly patterned (or in some cases unpatterned) lids in a parallel plate arrangement (Bouaidat et al., 2005, Lam et al., 2002, Nag et al., 2005, Zhao et al., 2002). Figure 1.1 demonstrates the approach utilized to develop such devices. Figure $1.1 \mathrm{~A}$ and $1.1 \mathrm{~B}$ demonstrate the configuration of a device fabricated with hydrophobic commodity polymers patterned on both bottom and top substrates with a hydrophilic silica 
sol gel material (Lam et al., 2002). Figure 1.1C exhibits the fluid front, determined through computational fluid dynamics, of a device (figure 1.1D) where the bottom substrate is composed of a hydrophilic borosilicate wafer patterned with hydrophobic plasmapolymerized hexafluoropropene barriers developed photolithographically. The top substrate in figure $1.1 \mathrm{C}$ and $1.1 \mathrm{D}$ is indiscriminately hydrophobic.

(A)

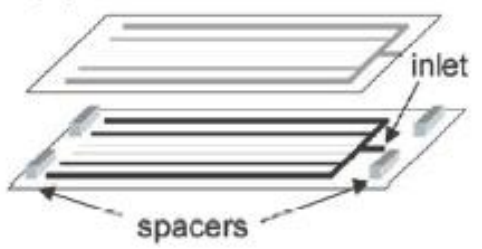

(C)
(B)

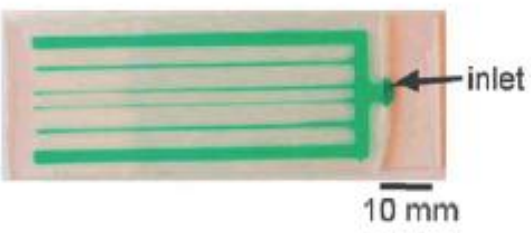

(D)

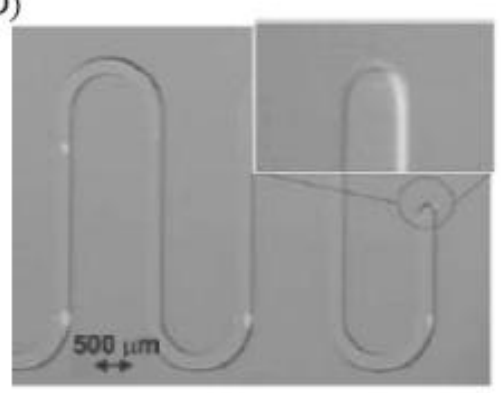

Figure 1.1 Surface tension-confined microfluidic device fabricated via sol gel processing (A) with a demonstration of the configuration of device and (B) the implementation of the device with colored fluid for visualization (adapted from Lam et al., Langmuir, 2002). (C) Computational fluid dynamic representation of the fluid front of a device fabricated with a patterned bottom substrate and indiscriminately hydrophobic top substrate. (D) The implementation of the device with an unpatterned top substrate (adapted from Bouaidat et al, Lab on a Chip, 2005).

Results from the development of these parallel plate surface tension-confined microfluidic have resulted in optimal device geometry recommendations. The optimal aspect ratio, defined as width per height of the device, has been determined through free energy 
considerations (Zhao et al., 2001). To ensure spontaneous capillary flow, the aspect ratio must follow Eq.(1.11) which indicates that the height of the device $b$, must always be less than the width of the device $a$, and the capacity to promote fluid flow is subject to the magnitude of the advancing contact angle of the hydrophilic path. The development of Eq.(1.11) follows arguments based upon the Laplace pressure drop associated with interfacial curvature.

$$
\frac{b}{a} \leq \cos \theta
$$

Consequently, as the advancing contact angle increases towards the $90^{\circ}$ limit for spontaneous flow, the capacity of the device to promote capillary flow diminishes drastically. This is demonstrated graphically by figure 1.2 .

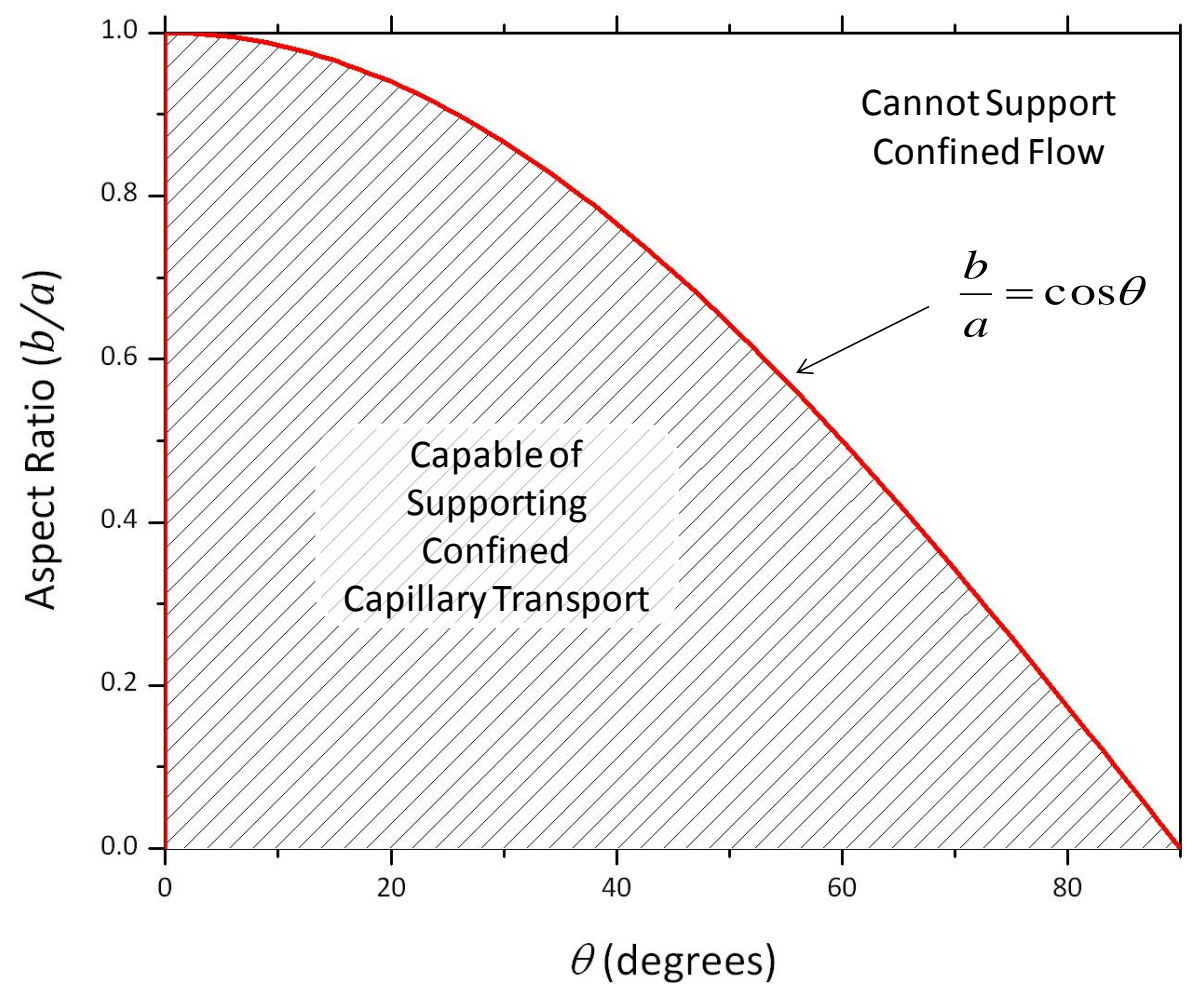

Figure 1.2 Geometrical limits facilitating confined capillary transport. 
Furthermore, devices have been developed demonstrating reactions and passive valves with self assembled monolayers of OTS via either multi-stream laminar flow or photolithography (Zhao et al., 2001, 2002). The pressure sensitive valves were demonstrated with silane solutions and water with trace amounts rhodamine-B for visualization purposes. The maximum pressure experienced based on capillarity was determined for these devices dependent upon device geometry, liquid surface tension $\gamma$ and the advancing contact angle for the non-polar hydrophobic region $\theta_{N}$.

$$
\Delta P_{\text {Max }}=\frac{2 \gamma}{b} \sin \left(\theta_{N}-\pi / 2\right)
$$

Furthermore, an application requiring a large interfacial surface area is demonstrated with an acidified gas and phosphate buffer neutralization in the presence of indicator. This investigation also presents conditions for critical width and curve radius enabling fluid transport under spontaneous conditions (Zhao et al., 2002). Furthermore, through energy minimization considerations, Lam independently derives and demonstrates Eq.(1.11) is in excellent agreement between theoretical and experimental results (Lam et al., 2002). Consequently, Eq.( 1.11) can be derived through an alternative but equally valid argument.

Another alternative system for the development of surface tension-confined microfluidic platforms has been established employing a significantly simplified fabrication process (Nag et al., 2005). The investigators develop devices through essentially painting hydrophobic materials upon a hydrophilic substrate. A reaction is incorporated into this system consisting of the combination of $\mathrm{FeCl}_{3}$ with $\mathrm{NH}_{4} \mathrm{SCN}$ to produce the bright red product $\mathrm{Fe}(\mathrm{SCN})_{3}$. These results indicate the limited rigor associated with manufacturing 
surface tension-confined microfluidic devices as well as the capacity to integrate reaction processes into an on-chip platform.

An additional method regarding the fabrication of surface tension-confined microfluidic devices entails chemically patterning plasma polymerized hexafluoropropene coating photolithographically upon a hydrophilic borosilicate wafer (Bouaidat et al., 2005). Consequently, such a drastic differential in wettability is established that only one patterned substrate is required and can be coupled with a hydrophobic top substrate. The advantage of this approach is that it eliminates the tedious necessity for proper alignment for top and bottom substrates. The investigators of this approach present a theoretical model to predict fluid front position versus time based upon a conservation of momentum approach.

$$
\frac{d\left(x^{2}\right)}{d t^{2}}+\frac{12 \mu}{\rho a^{2}} \frac{d\left(x^{2}\right)}{d t}+\frac{2 \gamma\left[2 a-b\left(\cos \theta_{1}+\cos \theta_{2}\right)\right]}{\rho a b}=0
$$

In this relation, $x$ denotes the fluid front position as a function of time $t$, system architecture $a$ and $b$, and intrinsic material properties including contact angles of the fluid upon the hydrophilic and hydrophobic materials, $\theta_{1}$ and $\theta_{2}$, respectively. The solution to this second order ordinary differential equation with respect to meniscus position squared can be solved relatively straightforward through numerical techniques. The solution determined by the investigators of this approach is in excellent agreement with experimental results. Furthermore, the investigators demonstrated that confluent monolayers of cells could be cultured in their apparatus and proteins could be selectively adsorbed upon the device surface (Bouaidat et al., 2005). Consequently, the surface tension-confined approach may be 
advantageous for applications requiring uninhibited gas exchange and surface sensitization for biocompatibility such as tissue engineering.

Lastly, the surface tension-confined approach is not without limitations. Due to the inherent simplicity associated with these devices, flow modulation and mixing become an issue. Moreover, bifurcations pose a challenge to flow control as fluid does not discriminate between paths to flow across. Preferentially controlling which path a particular fluid travels across is difficult, at best. One possible technique to mitigate this dilemma is to use mechanical actuation selectively to close paths inhibiting capillary pumping across paths that the user wishes to maintain dry given one substrate is not mechanically rigid (Vestad et al., 2004). In addition, flow within microfluidic devices tends to be rather laminar rendering convective mixing unfeasible. This aspect of micromixing becomes obvious when considering both the Reynolds and Peclet numbers as the characteristic length scale for microdevices is inherently minimal. However, one may be able to overcome this predicament by strategically patterning energetic resistance upon the device surface. This has been conducted effectively within the three-dimensional and electro-osmotically driven microfluidic analogs (Liu et al., 2005; Stroock et al., 2002 \& 2003) but has not yet been extended to the two-dimensional surface tension-confined technique.

Although much work still exists before chemically patterned devices provide novel solutions to problems in chemistry, biology and medicine, many of the reports discussed are encouraging regarding the capacity of surface tension-confined microfluidic devices to support autonomous chemical processes. 


\subsubsection{Curvature Modulation via Topographical Manipulation}

An alternative paradigm to facilitating capillary wicking via the surface tensionconfined technique includes deliberately manipulating the topography of a device to control interfacial curvature. This approach to surface tension-confined microfluidics generally exploits the fact that capillary-based flow can occur where the corners meet as a consequence of the diminishing radius of curvature at the intersection (Boys, 1959). However, for this flow to occur spontaneously, the Concus-Finn condition must be fulfilled considering the advancing contact angle $\theta$, as well as the corner half-angle $\alpha$ (Concus $\&$ Finn, 1969).

$$
\theta<\frac{\pi}{2}-\alpha
$$

This technique has been rigorously studied for the determination of equilibrated liquid morphology on structured surfaces such as rectangular channels and in v-grooves (Mann Jr et al., 1995, Romero \& Yost, 1996, Rye, Mann et al., 1996, Rye, Yost et al., 1996). In the case of rectangular channels, a map has been constructed enabling the a priori determination of ultimate liquid morphology based upon the contact angle of the substrate and aspect ratio of the duct cross-section (Seemann et al., 2005). Figure 1.3 illustrates this morphological dependency on fluid properties and system architecture. The associated syntax

addresses features characteristic of each morphological regime. Results were calculated theoretically with the SURFACE EVOLVER software package and were found to be in good agreement with experimental results investigated through AFM. 
(A)
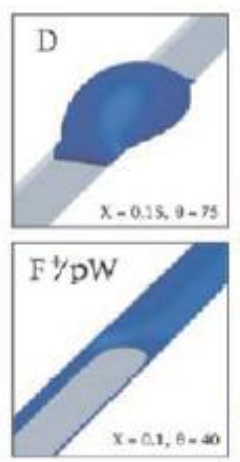

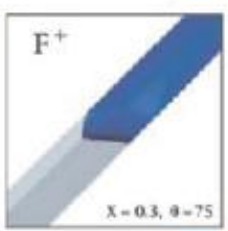

F 7pW

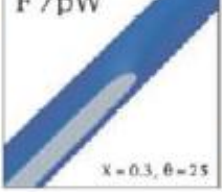

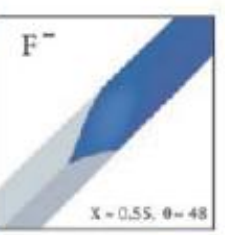

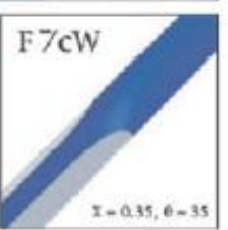

(B)

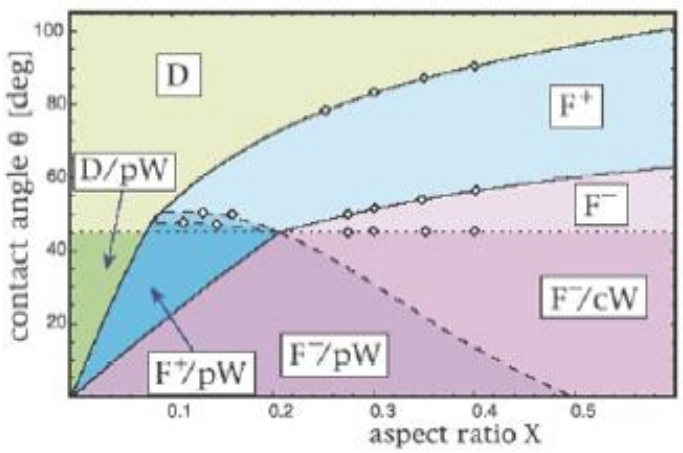

Figure 1.3 Demonstration of equilibrated liquid structure based on system geometry and advancing contact angle. Characteristic features include droplet (D), filament (F), pinnedwedges $(\mathrm{pW})$ and corner-wedges $(\mathrm{cW})$. The $+/$ - signs indicate whether the Laplace pressure associated with each morphology is positive or negative, respectively (adapted from Seemann et al., Proc. Nat. Acad. Sci., 2004).

Furthermore, the v-groove morphology has been thoroughly investigated and relations for capillary dynamics have been developed. Several alcohols with $\gamma / \mu$ ratios ranging from $46-970 \mathrm{~cm} / \mathrm{s}$ were studied in grooves of varying geometry machined into a copper substrate. Figure 1.4A illustrates the groove geometry while figure 1.4B demonstrates the experimental platform used to conduct the studies (Rye, Mann et al., 1996). Two models were developed characterizing the capillary dynamics. The first model considers a hydrodynamic approach where the driving force is a result of pressure drop across a curved interface. The second model depends strictly upon the total interfacial energy change while neglecting the shape of the curved interface. In both cases, flow was demonstrated to always follow a relation of the following form where $\mathrm{K}(\alpha, \theta)$ is a geometric parameter dependent on system geometry and contact angle.

$$
x=\left[\mathrm{K}(\alpha, \theta) h_{o} \frac{\gamma}{\mu} t\right]^{1 / 2}
$$


The results of these analyses demonstrate that the three-phase contact line location follows the square root of time. Coumarin fluorescent dye was utilized in small quantities to develop sufficient contrast of the three-phase line under UV irradiation for the comparison of experimental data to the models. The investigators of this approach conclude that exhaustive solutions to the capillary flow model can be realized; however, approximations via Eq.(1.15) can be employed for sufficiently accurate predictive models.

(A)

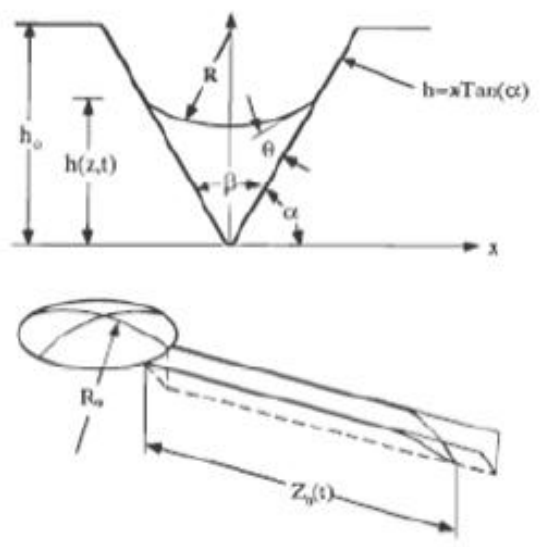

(B)

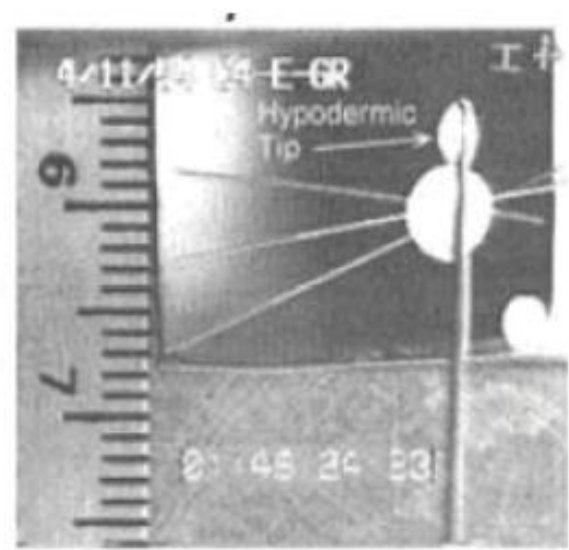

Figure 1.4 Illustration of v-groove experiments (A) with the demonstration of system architecture and (B) the experimental platform utilized in the study (adapted Rye et al., Langmuir, 1996).

As demonstrated through the referenced literature, topographical manipulation provides the capacity to induce capillary wicking through interfacial curvature modulation. As a result, one can exploit techniques such as photolithography, chemical etching and even three-dimensional machining to produce features promoting curvature management. Consequently, the approach of topographical manipulation is yet another straightforward but viable technique to produce surface tension-confined fluid transport. 


\subsubsection{Flow Modulation Techniques}

As already established in previous sections, the surface tension-confined approach to microfluidic system fabrication produces autonomously functioning devices with little rigor associated regarding implementation. This is a direct implication of the fact that the driving force for flow exploits capillary interactions producing spontaneous capillary flow. However, an unwanted artifact resulting from the inherent simplicity of these devices is that flow modulation is particularly difficult. This drawback is especially hindering for systems requiring the controlled introduction and the selective guidance of fluids across intricate geometry (e.g. on-chip ELISA). This section intends to introduce several techniques that have been or could be employed to guide fluids in specific directions.

One such mechanism to control flow directionality or rate includes manipulating thermocapillary shear stresses at the liquid air interface upon a substrate with a temperature gradient to facilitate fluid transport (Darhuber et al., 2006, Darhuber et al., 2003, Kataoka, 1999). This technique has been demonstrated through patterning a $\mathrm{SiO}_{2}$ wafer with hydrophobic strips of octadecyltrichlorosilane and coupling this substrate with a heater array to induce temperature gradients (Darhuber et al., 2006, Darhuber et al., 2003). The driving force for such thermocapillary induced flow is through local heating that can reduce the liquid surface tension producing thermocapillary shear stress, $\tilde{\tau} \cdot \hat{n}$ (Darhuber et al., 2003).

$$
\tilde{\tau} \cdot \hat{n}=\nabla \gamma=\frac{\partial \gamma}{\partial T} \nabla T
$$

For liquids, $\partial \gamma / \partial T$ is essentially temperature independent indicating that the driving force is $\nabla T$. This affect induces fluid transit from warmer areas to cooler areas of a substrate. 
Figure 1.5 demonstrates the capacity to control fluid progression utilizing this technique. As demonstrated, fluid can be directionally guided down required pathways and even across $90^{\circ}$ angles. Furthermore, models have been developed with a lubrication model approach and found to be in good agreement with the experimental results (Darhuber et al., 2003).

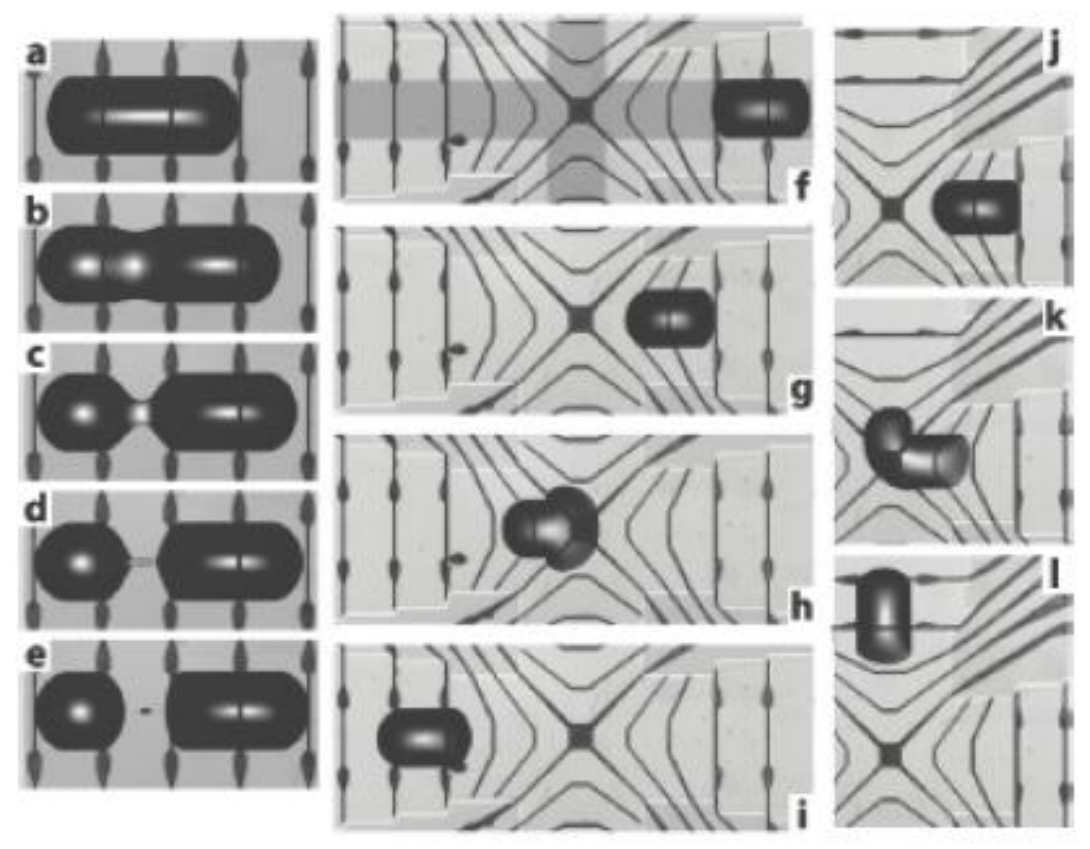

Figure 1.5 (a-e) Thermal induced division of a drop of dodecane on a selectively wetting strip $1000 \mu \mathrm{m}$ wide (elapsed time $=8.5$ seconds). (f-i) Dodecane drop propelled through a $1000 \mu \mathrm{m}$ wide intersection (elapsed time $=104$ seconds). $(\mathrm{j}-1)$ Dodecane drop thermally guided across a $90^{\circ}$ angle in a $1000 \mu \mathrm{m}$ wide intersection (elapsed time $=164$ seconds). Adapted from Darhuber et al., 2003.

Other techniques provide the potential to produce wettability modulation upon chemically patterned substrates. For example, 'contraphilic' polyurethane materials exist exhibiting an $83^{\circ}$ contact angle when dry but a $108^{\circ}$ contact angle in the presence of water (Makal \& Wynne, 2005). The proposed mechanism is that amide inter/intramolecular hydrogen bonding occurs in the dry and moderately hydrophilic state while surface-confined 
amide-water hydrogen bonding releases semifluorinated groups yielding a more hydrophobic surface when wet. This mechanism has been found to be reversible and could potentially serve as method to design passive gates between aqueous and non-aqueous solutions. This material could also be utilized to selectively pattern regions to impede fluid flowrate.

An additional example of a coating technique presenting a feasible solution to developing wettability modulation includes employing light sensitive compounds such as spiropyrans. These photoisomerizable compounds can undergo an $11-14^{\circ}$ reversible decrease in contact angle when light is switched from the visible region to ultraviolet (Garciá et al., , Rosario et al., 2002, Rosario et al., 2003). This photocapillary modulation technique could be utilized to control the Laplace pressure, and therefore the flowrate, in a microfluidic channel coated with spiropyran compounds.

Consequently, the field of flow modulation in surface-directed microfluidics is currently wide-open for investigation. Novel flow control methods are a necessity before integrating complex reaction processes into an on-chip format.

\subsection{Key Findings and Future Directions}

As demonstrated within this chapter, the surface tension-confined microfluidics approach offers a simple method for fabrication and implementation of micro-analytical systems. This burgeoning field offers great opportunity to resolve problems associated with more complicated microfluidic systems. Though this approach is rather new, several findings have already been established which may be of particular importance to investigators initiating research in this field. 
From a fluid physics perspective, rigorous solutions to the Navier-Stokes equations are not immediately realizable without sophisticated numerical approaches as a consequence of the poor understanding of slip velocities, precise tracking of the advection of the gas-fluid interface and establishment of surface tension or contact angle boundary conditions in capillary-based microfluidic systems. However, alternative approaches towards developing dynamic flow models indicate meniscus position progresses according to the square root of time and is dependent upon fluid/substrate properties and system geometry as demonstrated in Eq.(5). Interestingly, Mann et al. (Mann Jr et al., 1995, Romero \& Yost, 1996, Rye, Mann et al., 1996, Rye, Yost et al., 1996) found that a dynamic contact angle was not required to fit their experimental data to theoretical predictions. Furthermore, surface tension-confined microfluidic platforms can adequately be characterized as exhibiting low Reynolds, Péclet, capillary and Bond number flow. Subsequently, flow in surface-directed devices tends to be rather laminar with transport being dominated by diffusion with surface forces out-competing body forces such as gravity, respectively.

Chemical patterning and topographical manipulation techniques have successfully produced autonomous devices for fluid transport. In some cases, reaction processes have been integrated within these devices. However, this field is ripe with opportunity regarding the implementation of surface tension-confined platforms towards developing platforms that conduct reactive and diagnostic analyses. Preliminary diversion into theory has provided some relations that can be utilized for intelligent design pre-optimization; however, a great deal of investigation needs to be addressed before a comprehensive understanding of device function is established. 
Surface tension-confined microfluidic devices are inherently simple. Consequently, flow modulation is a concern. Methods have been employed to gain more control over flow such as mechanical actuation and thermocapillary induction. Moreover, material coatings provide the potential for the development of flowrate impediment and passive gate introduction through contraphilism or light-mediation.

The surface tension-confined microfluidics approach presents a manufacturing methodology involving minimal rigor. Furthermore, surface-directed devices are devoid of extraneous mechanical/electrical components which can lead to component malfunction and overall device failure. As a consequence of the inherent simplicity of surface tensionconfined microfluidic devices, implementation of the platform is straightforward and requires limited analytical experience. However, before wide-scale deployment of surface tensionconfined microfluidic devices can be realized, a more comprehensive understanding of the underlying physics needs to be conducted. Certain phenomena play a larger role in fluid physics as liquid samples approach the nanoliter scale. For example, long-ranged intermolecular interactions, thermal fluctuations such as capillary waves and the influence of the molecular structure of the fluid all inherit a greater capacity to affect fluid physics as surface area to volume ratios decrease (Dietrich et al., 2005). To realize greater degrees of parallelization and high-throughput, a better theoretical framework is a necessity regarding microfluidics as a whole encompassing the surface tension-confined technique (Hong \& Quake, 2003). Lastly, fluid sample pretreatment techniques are of great consideration to ensure reproducible and reliable function of devices as only minimal amounts of contaminants are required to influence capillary dynamics in surface tension-confined devices. A comprehensive literature review exists as a basis for microfluidic sample 
pretreatment (Lichtenberg et al., 2002); however, these findings need to be extended to the surface tension-confined approach to enhance platform reliability. Addressing such concerns may expedite the application of the surface tension-confined microfluidics towards current problems in chemistry, biology and medicine.

\subsection{References}

Adamson, A. W. \& Gast, A. P. (1997). Physical Chemistry of Surfaces, 6-thed. WileyInterscience: New York.

Bouaidat, S., Hansen, O., Bruus, H., Berendsen, C., Bau-Madsen, N. K., Thomsen, P., Wolff, A. \& Jonsmann, J. (2005). Lab on a Chip 5, 827-836.

Boys, C. V. (1959). Soap Bubbles: Their Colours and the Forces which Mold Them: Being the Substance of Many Lectures Delivered to Juvenile and Popular Audiences: with the Addition of Several New and Original Sections. Dover Publications.

Brakke, K. A. (1992). Experimental Mathematics 1, 141-165.

Burns, M. A. (1998). Science 282, 484-487.

Choban, E. R., Markoski, L. J., Wieckowski, A. \& Kenis, P. J. (2004). Journal of Power Sources 128, 54-60.

Concus, P. \& Finn, R. (1969). Proceedings of the National Academy of Sciences 63, 292299.

Darhuber, A. A., Troian, S. M., Miller, S. M. \& Wagner, S. (2006). Journal of Applied Physics 87, 7768-7775.

Darhuber, A. A., Troian, S. M. \& Reisner, W. W. (2001). Physical Review E 64, 31603.

Darhuber, A. A., Valentino, J. P., Davis, J. M., Troian, S. M. \& Wagner, S. (2003). Applied Physics Letters 82, 657-659.

Dietrich, S., Popescu, M. N. \& Rauscher, M. (2005). Journal of Physics: Condensed Matter 17, S577-S593.

Garciá, A. A., Cherian, S., Park, J., Gust, D., Jahnke, F. \& Rosario, R. Letters.

Gau, H., Herminghaus, S., Lenz, P. \& Lipowsky, R. (1998). Phys. Rev. Lett 81, 1253. 
Henriksson, U. \& Eriksson, J. C. (2004). Journal of Chemical Education 81, p1505.

Hong, J. W. \& Quake, S. R. (2003). Nature Biotechnology 21, 1179-1183.

Kamholz, A. E., Schilling, E. A. \& Yager, P. (2001). Biophysical Journal 80, 1967-1972.

Kataoka, D. E. (1999). Nature 402, 794-797.

Lam, P., Wynne, K. J. \& Wnek, G. E. (2002). Langmuir 18, 948-951.

Lichtenberg, J., de Rooij, N. F. \& Verpoorte, E. (2002). Talanta 56, 233-266.

Liu, A., He, F., Wang, K., Zhou, T., Lu, Y. \& Xia, X. (2005). Lab on a Chip 5, 974-978.

Makal, U. \& Wynne, K. J. (2005). Langmuir 21, 3742-3745.

Mann Jr, J. A., Romero, L., Rye, R. R. \& Yost, F. G. (1995). Physical Review E 52, $3967-$ 3972.

Nag, A., Panda, B. R. \& Chattopadhyay, A. (2005). Pramana Journal of Physics 65.

Oh, C. S. (1999).

Romero, L. A. \& Yost, F. G. (1996). Journal of Fluid Mechanics 322, 109-129.

Rosario, R., Gust, D., Hayes, M., Jahnke, F., Springer, J. \& Garcia, A. A. (2002). Langmuir $18,8062-8069$.

Rosario, R., Gust, D., Hayes, M., Springer, J. \& Garcia, A. A. (2003). Langmuir 19, 88018806.

Rye, R. R., Mann, J. J. A. \& Yost, F. G. (1996). Langmuir 12, 555-565.

Rye, R. R., Yost, F. G. \& Mann Jr, J. A. (1996). Langmuir 12, 4625-4627.

Sato, K., Yamanaka, M., Hagino, T., Tokeshi, M., Kimura, H. \& Kitamori, T. (2004). Lab on a Chip 4, 570-575.

Seemann, R., Brinkmann, M., Kramer, E. J., Lange, F. F. \& Lipowsky, R. (2005). PNAS $102,1848-1852$.

Squires, T. M. \& Quake, S. R. (2005). Reviews of Modern Physics 77, 977-1026.

Vestad, T., Marr, D. W. M. \& Oakey, J. (2004). Journal of Micromechanics and Microengineering 14, 1503-1506. 
Washburn, E. W. (1921). Physical Review 17, 273-283.

Wilkes, J. O. (2006). Microfluidics and Electrokinetic Flow Effects, Fluid Mechanics for Chemical Engineers with Microfluidics and CFD, pp. 639-665. Upper Saddle River: Prentice Hall.

Zhao, B., Moore, J. S. \& Beebe, D. J. (2001). Science 291, 1023-1026.

Zhao, B., Moore, J. S. \& Beebe, D. J. (2002). Anal. Chem 74, 4259-4268. 


\section{Chapter 2: Fundamentals of Flow in a Microfluidic Regime}

\subsection{Surface Tension and Surface Free Energy}

As length scales decrease in a microfluidic regime, the domination of body forces (e.g. gravity) begins to subside while surface forces (i.e. surface tension in particular) become more important aspects of fluid physics and transport. Consequently, it is important to have an understanding of how surface tension and material surface energy can influence wettability and dynamic spreading of a fluid upon a surface. Especially important, with regard to surface tension-confined microfluidics (STCM), is understanding how abrupt disparities in surface energy, and therefore contact angle, affect the overall curvature of a fluid interface on that surface. Developing such an understanding enables intelligent design and platform pre-optimization. Furthermore, an understanding of the critical parameters affecting capillary transport also lends insight into device limitations and opportunities for improvement of the STCM technology.

\subsection{Surface Tension Dependency on System Properties}

As the flow and confinement within the surface tension-confined microfluidic device is a result of the high surface tension that aqueous fluids exhibit, it is necessary to explore aspects that can influence the surface tension of a fluid. Such factors include, but are not limited to, the contact angle of a fluid on a substrate, the temperature and pressure of a system, the composition of the system, and the surface area to volume ratio (or interface to bulk ratio) experienced within the STCM device. Below is a description of how each 
phenomenon affects surface tension as well as brief discussion as to how important such influences are on the scale of microfluidic platforms fabricated within this manuscript addressing ambient laboratory conditions.

\subsubsection{Surface tension expansion via contact angle}

First and foremost, an adequate understanding of surface tension is required for the exploitation of autonomous fluid transport within the STCM device. Surface tension, defined in units of force per unit Cartesian length (commonly dynes/cm or $\mathrm{mN} / \mathrm{m}$ ), is the degree of affinity a solution has intermolecularly with itself as opposed intermolecular affinity with a surrounding bath of a fluid of different composition (Fisher et al., 1982). This measure can be thought of intuitively as a measure of force required to stretch a film of constant thickness of a material a defined length. It is sometimes more convenient to consider surface energy (or surface free energy) of a pure fluid which is the two-dimensional analog to surface tension measured in force per unit area (e.g. ergs $/ \mathrm{cm}^{2}$ or $\mathrm{J} / \mathrm{m}^{2}$ ). Although surface free energy and surface tension are mathematically equivalent, it is perhaps more intuitive to consider surface energy as the magnitude of work required to bring a molecule from the interior of a fluid to the interface rather than a tension associated with a theoretical 'skin' surrounding a fluid element (Adamson \& Gast, 1997). Discussed in the following section is the dependency of surface tension, and thus surface free energy, on factors such as temperature and pressure as well as a discussion on the characterization of surface tension via contact angle and the importance of maintaining adequate rigor in such a calculation.

As previously mentioned, it is possible to expand the surface tension, $\gamma$, of a fluid via the contact angle that the fluid makes when in intimate contact with a solid in the presence of 
a known gas phase. The contact angle, $\theta$, is defined as the angle between the fluid and the solid material at the three-phase contact point (in $\mathbb{R}^{2}$ ) or line (in $\mathbb{R}^{3}$ ). For complete wetting, $\theta=0$, a fluid spreads horizontally along a surface. Furthermore, Fisher et al., indicate that for small $\theta$, the following expansion can be utilized to calculate surface tension, $\gamma$ (Fisher $e t$ al., 1982).

$$
\gamma(\theta)=\gamma(0)+\frac{1}{2} \gamma^{\prime \prime}(0) \theta^{2}+\cdots
$$

For this expression, a 2-dimensional Ising model is implied and consequently, the linear term of the expansion does not exist since the roughening temperature for a 2D Ising model is zero (Herring, 1953, Leamy et al., 1975). For a macroscopic system of a given width, L, the length of the interface is given by $L \sec \theta$ and the free energy change, $\Delta F$, resulting from a distortion of the interface is given by the following.

$$
\Delta F=\gamma(\theta) L \sec \theta-\gamma(0) L=\frac{1}{2} L \theta^{2}\left[\gamma(0)-\gamma^{\prime \prime}(0)\right]
$$

The resistance to small distortions is thus an effective interfacial tension, $\Gamma$, and comes directly from the free energy response to a distortion (Fisher et al., 1982).

$$
\Gamma=\gamma(0)-\gamma^{\prime \prime}(0)
$$

As temperature decreases, $\gamma^{\prime \prime}(0)$ quickly diverges while $\gamma(0)$ remains relatively constant. Consequently, it becomes necessary to consider the correction term.

The application of simple capillary wave theory enables the determination of the interfacial profile considering that the fluid is below the critical temperature, $\varpi^{2}=$ $L / \sinh (\gamma(0) / T$ ) (Abraham, 1981, Buff et al., 1965). However, substituting equation 2.3 into this relation yields a new expression utilizing the correction term. 


$$
\varpi^{2}=\frac{L T}{\gamma(0)-\gamma^{\prime \prime}(0)}
$$

The exact result for capillary wave theory has been calculated (Abraham \& Reed, 1977) yielding the exact result $\sinh (\gamma(0) / T)=T^{-1}\left[\gamma(0)-\gamma^{\prime \prime}(0)\right]$ demonstrating that the exact result is in precise agreement with the generalized capillary wave theory as demonstrated by Fisher et al. (Fisher et al., 1982). The above method indicates that the contact angle for a material system can be utilized to characterize the system surface tension. Furthermore, it is necessary to be rigorous and carry a correction factor defining an effective surface tension when analyzing parameters that are dependent upon surface tension and temperature such as the second moment of the interface profile. This result does not enable device design optimization but rather indicates the utility of generalized capillary wave theory when expanding surface tension via contact angle.

\subsubsection{Surface tension dependency on area per volume}

The volume of fluid with respect to surface area can also have an effect on surface tension. Bulk to surface ratio is particularly interesting for the microfluidic regime as this is an intrinsic property of microfluidic systems. Accordingly, the following section seeks to explicate the relationship of fluid volume to surface area by the decomposition of volume to the product of a characteristic length scale and surface area. Possibly the most theoretically basic system that can be considered is a droplet of water surrounded by air. Furthermore, to simplify the problem, the air-water vapor phase is considered to be ideal and partial pressures are considered as constant throughout the system. Although the spherical geometry is not necessarily applicable to the parallel plate microfluidic systems described, this approach still 
lends insight into the length scales when surface tension becomes a function of bulk versus boundary layer energetics.

In order to demonstrate this phenomenon, it is required to take a step back and properly define our system of interest. As a consequence, this will enable the establishment of the formal thermodynamical framework necessary to resolve a solution. Specifically, for a system represented by figure 2.1, a density profile versus droplet radial position, $x$, for water in air is presented. From a mechanical standpoint, the system is considered to behave as two homogenous fluids separated by an infinitesimally thin and uniform film or membrane (Defay \& Prigogine, 1966).

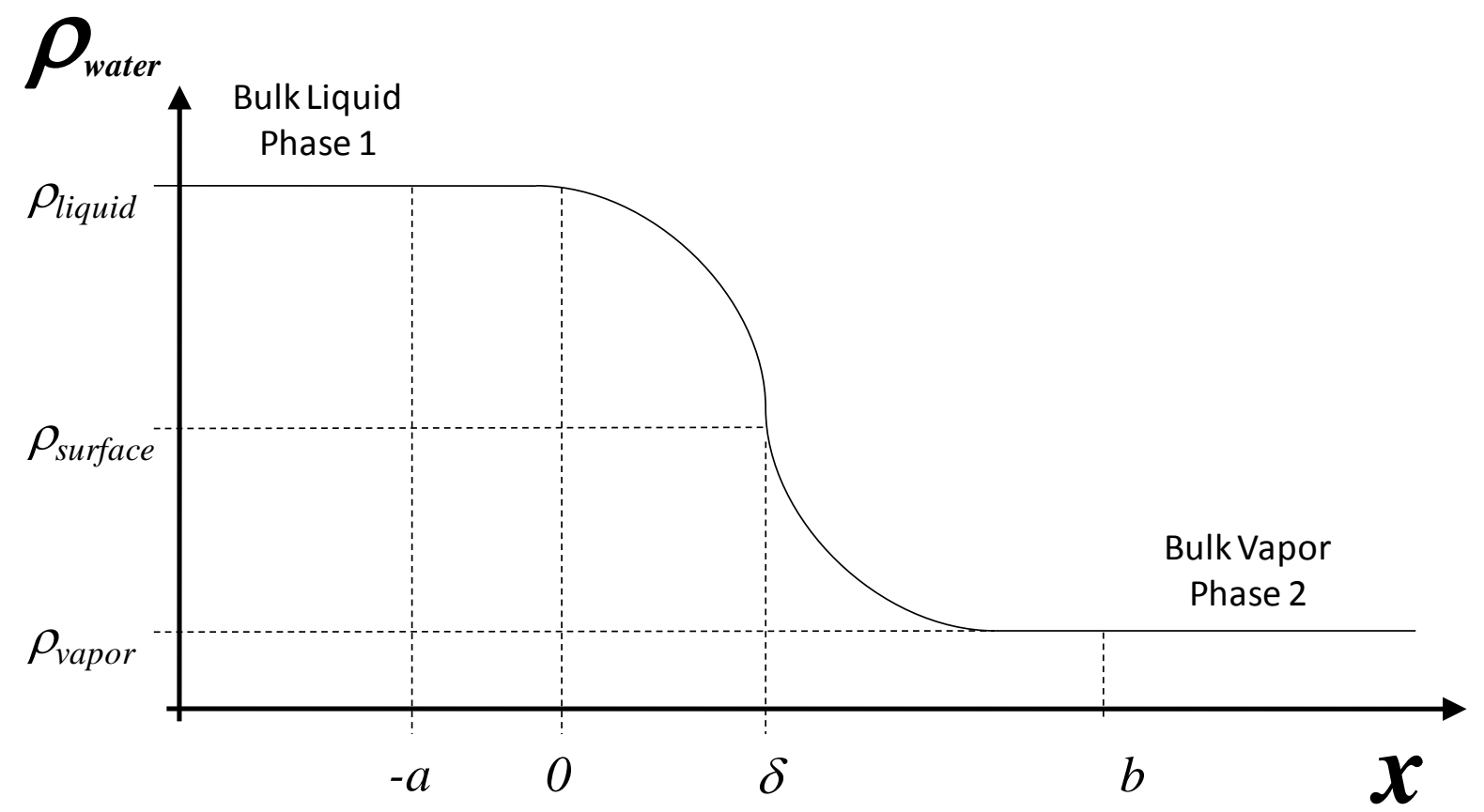

Figure 2.1 Density profile of water versus radial position in a droplet suspended in air.

Location $-a$ and $b$ denote the radial positions in which the density is sufficiently homogenous. Location $x=0$ is where the surface under tension begins and location $\delta$ is where 
the density excess, to be rigorously defined later, is exactly zero. Considering isothermal conditions and eliminating the possibility of reactive processes by system simplification to strictly water and air components (implicitly representing nitrogen, oxygen, and the other components of air as an abbreviation), the system illustrated by the density profile in figure 2.1 can be thermodynamically specified by the temperature $T$, the interfacial area $A$, the volume of water and air in phases 1,2 , and on the surface $\left(V_{w}{ }^{1}, V_{w}{ }^{2}, V_{w}^{s}, V_{a}{ }^{l}, V_{a}{ }^{2}, V_{a}^{s}\right.$, respectively), and the moles of water and air in phases 1,2 , and on the surface $\left(n_{w}{ }^{1}, n_{w}{ }^{2}, n_{w}{ }^{s}\right.$, $n_{a}{ }^{l}, n_{a}{ }^{2}, n_{a}{ }^{s}$, respectively). It directly follows that the variation in the internal energy balances in phases 1 and 2 as well as on the surface, $s$.

$$
\begin{array}{ll}
d U^{1}=T d S^{1}+\gamma d A+\mu_{w} d n_{w}^{1}+\mu_{a} d n_{a}^{1} & 2.5 \\
d U^{2}=T d S^{2}+\gamma d A+\mu_{w} d n_{w}^{2}+\mu_{a} d n_{a}^{2} & 2.6 \\
d U^{s}=T d S^{s}+\gamma d A+\mu_{w} d n_{w}^{s}+\mu_{a} d n_{a}^{s} & 2.7
\end{array}
$$

The surface tension term is represented via $\gamma$ and $\mu_{i}$ indicates the chemical potential of the $i$ component of the system. In relations $2.5-2.8$, the abbreviated term for air is actually a sum of the components of air, $c$, from component 1 to $n$ considering nitrogen, oxygen, and other gases $\left(\mu_{a} d n_{a}=\sum_{c=1,2 \ldots}^{n} \mu_{c} d n_{c}\right)$.

Since the surface is of particular interest, the following Gibbs-Duhem equation can be derived by integrating expression 2.7 and subsequently taking the derivative of the integral and subtracting it again from expression 2.7 .

$$
0=S^{s} d T+A d \gamma+n_{w}^{s} d \mu_{w}+n_{a}^{s} d \mu_{a}
$$


As previously mentioned, in order to idealize the system, the derivation assumes the system is isothermal, and the composite gas is perfect with constant partial pressure. As a result of the assumptions, relation 2.8 decomposes to the following expression.

$$
d \gamma=-\Gamma_{w}^{s} d \mu_{w}
$$

Here $\Gamma_{w}^{s}$ is $n_{w}^{s} / A$ and is defined as the surface excess concentration of water at the interface (units of moles per area) and $\mu_{w}$ is the chemical potential of water on the surface. Furthermore, in conformity with the general equation of Gibbs (Gibbs, 1906) for the dependence of the pressure of a homogenous phase on its state, it is possible to write the following relations under the assumptions of isothermal and a static, equilibrated, system (Tolman, 2004).

$$
d \mu_{w}=\frac{d p^{1}}{\rho^{1}}=\frac{d p^{2}}{\rho^{2}}
$$

In relation $2.10, d p^{i}$ represents the variation of pressure for phase $i$ and $\rho^{i}$ denotes the density for phase $i$.

In addition, the pressure jump across a curved interface for a spherical droplet is known (Adamson \& Gast, 1997).

$$
p^{1}-p^{2}=\frac{2 \gamma}{R}
$$

Where $R$ is the radius of the sphere and $c=1 / R$ is the mean curvature of the system $(\Delta p=2 \gamma c)$. Consequently, the curvature-based pressure difference from the bulk liquid across to the vapor phase is now established since the mean curvature for the system is known. The determination of mean curvature for more complicated geometries will be discussed in later sections of this compilation. 
From this point, expression 2.10 can be substituted into 2.9 and through the subsequent rearrangement, an expression for surface tension versus pressure can be determined.

$$
\begin{gathered}
d \gamma=\frac{-\Gamma_{w}^{S}}{\left(\rho^{1}-\rho^{2}\right)} d\left(p^{1}-p^{2}\right) \\
d \gamma=\frac{-\Gamma_{w}^{S}}{\left(\rho^{1}-\rho^{2}\right)} d\left(\frac{2 \gamma}{R}\right)
\end{gathered}
$$

Or following simplification of expression 2.13, the resulting differential equation representing the surface tension dependency on spherical radius can be derived for the isothermal system at equilibrium as demonstrated by Tolman et al.

$$
\frac{1}{\gamma} \frac{d \gamma}{d R}=\frac{\left(2 / R^{2}\right)\left[\Gamma_{w}^{s} /\left(\rho^{1}-\rho^{2}\right)\right]}{\left[1+(2 / R)\left[\Gamma_{w}^{s} /\left(\rho^{1}-\rho^{2}\right)\right]\right]}
$$

Tolman, et al., describe $\Gamma_{w}^{S}$ as the difference per unit of dividing area between the actual amount of water (or any general liquid) in the system and that of a system with a stepchange in density across the interface rather than the sigmoidal change proposed in figure 2.1. Using this definition, Tolman, et al., develop the following integral with respect to $x$ (the radial distance from the center of the sphere) with the representation of curvature as $c=$ $1 / R$

$$
\Gamma_{w}^{S}=\int_{-a}^{0}\left(\rho-\rho^{w}\right)(1+c x)^{2} d x+\int_{0}^{b}\left(\rho-\rho^{a}\right)(1+c x)^{2} d x
$$

Here the limits of integration are from the position of liquid bulk density, $-a$, to the beginning of the surface of tension, $x=0$; and from the surface of tension again to the position of gas bulk density, $b$. 
However, if the limits of integration are changed, one can consider the position in which the surface excess concentration is equal to zero.

$$
0=\int_{-a}^{\delta}\left(\rho-\rho^{w}\right)(1+c x)^{2} d x+\int_{\delta}^{b}\left(\rho-\rho^{a}\right)(1+c x)^{2} d x
$$

Finally, expression 2.16 can be expanded into the following integral.

$$
\begin{aligned}
0=\int_{-a}^{0}\left(\rho-\rho^{w}\right) & (1+c x)^{2} d x+\int_{0}^{\delta}\left(\rho-\rho^{w}\right)(1+c x)^{2} d x \\
& +\int_{0}^{b}\left(\rho-\rho^{a}\right)(1+c x)^{2} d x-\int_{0}^{\delta}\left(\rho-\rho^{a}\right)(1+c x)^{2} d x
\end{aligned}
$$

Tolman, et al., arrive at the final relation via subtracting 2.17 from 2.15 and the subsequent integration.

$$
\begin{gathered}
\Gamma_{w}^{s}=\int_{0}^{\delta}\left(\rho^{w}-\rho^{a}\right)(1+c x)^{2} d x \\
\frac{\Gamma_{w}^{S}}{\left(\rho^{w}-\rho^{a}\right)}=\delta+c \delta^{2}+\frac{1}{3} c^{2} \delta^{3}
\end{gathered}
$$

Expression 2.19 can be substituted directly into expression 2.14 and assuming $c \delta^{2}$ and $c^{2} \delta^{3}$ are small in comparison to $\delta$, the result can be integrated to arrive at an expression for surface tension with respect to radius and the width of the surface of tension, $2 \delta$ (Tolman, 2004).

$$
\frac{\gamma}{\gamma_{o}}=\frac{1}{(1+2 \delta / R)}
$$

Here, $\gamma_{o}$ represents the surface tension value of a fluid droplet with a radius that tends to infinity (i.e. the surface becomes a plane). Expression 2.20 thereby yields a relation that describes the surface tension variation between a plane and a droplet with a given radius at a 
constant temperature and equilibrated state. Although expression 2.20 has been derived explicitly for spherical geometry, the final result from this exercise is that surface tension decreases substantially as characteristic length scale approaches the order of $\delta$. As $\delta$ is on the order of molecular lengths, this effect is manifested much less in a microfluidic regime rather than a nanofluidic regime.

The same derivation developed by Tolman, et al., can be directly applied to the Cartesian STCM geometry. The derivation is essentially the same with the exception that the curvature term ( $c=1 / R$ in the spherical case) is altered for the Cartesian system.

$$
c=\left[\frac{\cos \theta_{P}}{h}+\frac{\cos \theta_{N}}{w}\right]
$$

Where $h$ and $w$ represent the channel height and path width, respectively, and $\theta_{P}$ and $\theta_{N}$ represent the contact angle of the fluid component upon the polar (hydrophilic) and non-polar (hydrophobic) substrate materials (Delamarche et al., 1998, Juncker et al., 2002). Material systems and associated contact angles will be discussed in detail in chapter 3 . As a result, the Cartesian analog to the spherical relationship for surface tension dependency versus characteristic length is easily determined, viz.

$$
\frac{\gamma}{\gamma_{o}}=\frac{1}{\left[1+2 \delta\left(\frac{\cos \theta_{P}}{h}+\frac{\cos \theta_{N}}{w}\right)\right]}
$$

Although length scales currently mandated by current fabrication procedures are far from approaching the limits where fluid surface tension become susceptible to interfacial energetics, relation 2.22 yields a relation demonstrating that the driving force for spontaneous flow decreases at small length scales. Such theoretical results must be kept in mind as microfluidics decreases to the scale of nanofluidics. 


\subsubsection{Thermal influence on surface tension}

Surface tension can be manipulated through temperature gradients as alluded to in chapter 1 (relation 1.16). Specifically, thermo-capillary shear stresses can be initiated through temperature gradients. This effect was first observed by Volkoviski when watching the propensity of a liquid film to spread toward the cooler side of an unevenly heated substrate (Volkoviski, 1935).

The governing equation of thermocapillary induction is the convection-diffusion equation below. Specifically, the convection-diffusion equation can be implemented to track the movement of a set of molecules in a multi-component system over time $t$.

$$
\frac{\partial c}{\partial t}+\boldsymbol{u} \cdot \nabla c=D \nabla^{2} c
$$

Where $c$ is the concentration of a particular species, $\boldsymbol{u}$ is the three component velocity vector and $D$ is the diffusion coefficient of species $c$. Furthermore, relation 2.23 must be coupled with the velocity field with contributions from thermocapillary shear stresses and capillarity.

$$
\boldsymbol{u}=\frac{1}{\mu}\left[\tau z+\left(\frac{z^{2}}{2}-z h(x, y, t)\right) \nabla p\right]
$$

In relation $2.24, \mu$ represents viscosity, $h$ is the maximum film location for a particular $x, y$, and $t$ (for example, $h$ is a Monge surface representation of the interface discussed more comprehensively in chapter 3). Moreover, $\tau$ is the thermocapillary stress based on temperature $T$ represented as $\tau \equiv(\partial \gamma / \partial T) \cdot \nabla T$ and the capillary pressure drop is represented as $\nabla p=-\nabla\left(\gamma \nabla^{2} h\right)$. In the limit where the length of a fluid duct becomes very large with respect to height, the spatial difference in surface tension can be ignored (i.e. if 
$(h / L)^{2} \ll 1, \nabla p \approx-\gamma \nabla\left(\nabla^{2} h\right)$ (Darhuber, Davis et al., 2003). Consequently, relation 2.24

now becomes the following.

$$
\boldsymbol{u}=\frac{1}{\mu}\left[\left(\frac{\partial \gamma}{\partial T} \cdot \nabla T\right) z-\gamma\left(\frac{z^{2}}{2}-z h\right) \nabla\left(\nabla^{2} h\right)\right]
$$

This phenomenon has recently garnered attention from the microfluidics community as a means to manipulate minute quantities of liquid. Thermal gradients can be generated on a substrate surface through embedded microheaters or by radiative heating from above (Hitt \& Smith 1993, Schatz et al. 2002). Darhuber, et al., (2003a) studied the horizontal transport of fluid filaments traveling across a chemically-patterned substrate and proposed kinetics describing the fluid transport. In addition, Darhuber, et al., also published a review discussing alternative means in which surface stresses can be exploited as techniques for fluid manipulation (Darhuber \& Troian, 2005). Grigoriev, et al., developed a means to study mixing within microdroplets undergoing transport within a fluid substrate subject to a thermal gradient (Grigoriev, 2005). As mixing in spherical droplets is governed by symmetries, the formation of invariant surfaces occurs acting as an effective barrier to transport and efficient mixing. As a result, Grigoriev, et al., investigated three types of symmetry occurring in spherical droplets and proposed a means in which to optimize mixing within microdroplets. Darhuber, et al., also investigated thermocapillary-driven mixing finding that three regimes seem to exist based on the Péclet number, Pé $=U L / D$, with regard to mixing efficiency where $U$ is a characteristic velocity, $L$ is the characteristic length scale and $D$ is the system diffusion constant (Darhuber, 2002, Darhuber, Davis et al., 2003, Darhuber \& Troian, 2005, Darhuber, Valentino et al., 2003). Jiao, et al., demonstrated the same approach in a closed system via the manipulation of liquid plugs in a glass capillary 
through thermocapillary induction (Jiao et al., 2007). In this study, Jiao, et al., reveal that a fluid plug can undergo reciprocating motion in a capillary tube via examining the coupling of heat transfer and fluid mechanics. A one-dimensional model is posed describing this effect yielding the conclusion that greater reciprocation frequencies and higher fluid viscosities results in a decrease in distance that the fluid plug travels during each cycle.

Surface tension-confined microfluidics does not directly take advantage of temperature gradients for fluid transport manipulation. However, since some of the fabrication approaches (discussed thoroughly in chapter 3) are the same techniques utilized to make microheater systems, there is an opportunity to couple the developed platforms with heating equipment to gain further control over fluid transport. At the time of writing this document, no experiments have been performed taking advantage of such techniques.

\subsubsection{Surface tension versus pressure}

The effect of pressure on surface tension, $(d \gamma / d p)$, for a multiphase system has been scrutinized since first proposed by Gibbs nearly a century ago (Gibbs, 1906). The theoretical implication of this quantity has been studied through a variety of contexts. Lewis and Randall discussed to the Maxwell relation of this quantity (Lewis \& Randall, 1923).

$$
\left(\frac{\partial \gamma}{\partial p}\right)_{T, A}=\left(\frac{\partial V}{\partial A}\right)_{T, p}
$$

Defay and Priogione realized such a quantity is related to a length as the result of a ratio of volume to area (Defay \& Prigogine, 1966). Understanding the relationship of pressure on surface tension is important for systems with dynamic pressure gradients. For example, currently the pressure dependency on surface tension is of great interest for the understanding 
of pulmonary systems and the cause of edema (Albert et al., 1979, Bachofen et al., 1970, Bachofen et al., 1987). However, a great deal of argument has ensued as to the proper establishment of the Maxwell relation 2.26.

Starting from the variation of Gibbs free energy for the two-component water-air system one can derive the Maxwell relations.

$$
d G=-S d T+V d p+\mu_{w} d n_{w}+\mu_{a} d n_{a}+\gamma d A
$$

From expression 2.27, we can find equilibrium relations for both volume $\left[V=(d G / d p)_{A, T, n_{w} n_{a}}\right]$ and surface tension $\left[\gamma=(d G / d A)_{p, T, n_{w} n_{a}}\right]$. Consequently, the Maxwell relation is a direct result.

$$
\left(\frac{\partial \gamma}{\partial p}\right)_{T, A, n_{w}, n_{a}}=\left(\frac{\partial V}{\partial A}\right)_{T, p, n_{w}, n_{a}}
$$

Although expression 2.28 is in fact valid, Mann and Turkevich note that it is rather awkward as a result of combining both extensive and intensive properties. In addition, Mann and Turkevich use a Legendre transformation to construct a new and equivalent potential strictly dependent upon intensive properties $\Xi\left(T, p, \mu_{i}, S\right)=G\left(T, p, n_{i}, S\right)$. From the new potential, an alternative Maxwell relation is realized (Turkevich \& Mann, 1990).

$$
\left(\frac{\partial \gamma}{\partial p}\right)_{T, A, \mu_{w}, \mu_{a}}=\left(\frac{\partial V}{\partial A}\right)_{T, p, \mu_{w} \mu_{a}}
$$

Regarding the binary system of gas-liquid at standard pressure and temperature conditions, the chemical potentials are completely determined and it is possible to consider relation 2.26 as the correct Maxwell relation. 
Moreover, Mann and Turkevich use a Cahn-Hilliard density gradient formalism to obtain an explicit relationship for the pressure dependence on surface tension for a planar surface within inhomogeneous mean field theory thus obviating the need to specify the Gibbs interface between two fluid phases (Turkevich \& Mann, 1990).

$$
\left(\frac{\partial \gamma}{\partial p}\right)_{T, A}=\left[\frac{\left(\frac{\delta n_{g}}{\delta c}\right)_{c_{o}}\left(\frac{\delta^{2} n_{l}}{\delta c^{2}}\right)_{c_{o}}-\left(\frac{\delta^{2} n_{g}}{\delta c^{2}}\right)_{c_{o}}\left(\frac{\delta n_{l}}{\delta c}\right)_{c_{o}}}{n_{g}\left(c_{o}\right)\left(\frac{\delta n_{l}}{\delta c}\right)_{c_{o}}-n_{l}\left(c_{o}\right)\left(\frac{\delta n_{g}}{\delta c}\right)_{c_{o}}}\right]\left(\Delta c_{o}\right)^{2} L
$$

Here, $n_{i}$ is the molar volume of component $i$ and $c$ is the composition (specified as mole fractions) where $c_{o}$ is the critical composition near the critical temperature and $\Delta c_{o}$ is defined as $\Delta c_{o}=c_{g}-c_{o}=c_{o}-c_{l}$. Finally, $L$ is the length of the interface (referencing figure 2.1, $L=2 \delta$ where $\delta$ is the surface half-width rather than the differential operator as used in relation 2.7). Hence a change in pressure under isothermal and constant surface area conditions results in phase partitioning thereby altering the surface tension of the fluid component.

Because interface width is very small, most likely several molecular lengths of water, this effect does not manifest itself very drastically over the narrow range of ambient pressures experienced in the laboratory. Subsequently, the effect of pressure on surface tension is important to be aware of but not of critical concern in the design of surface tension-confined microfluidic platforms. 


\subsection{Young's Equation and Contact angle measurement}

In most instances, as a drop of fluid is placed on a substrate, the fluid will not completely wet the solid substrate forming an angle between the solid surface and the fluid (see figure 2.2). This angle, known as the contact angle, is commonly used as a measure of affinity that a fluid has for a substrate and can be directly related to the energetic interactions of the substrate and fluid as will be demonstrated.

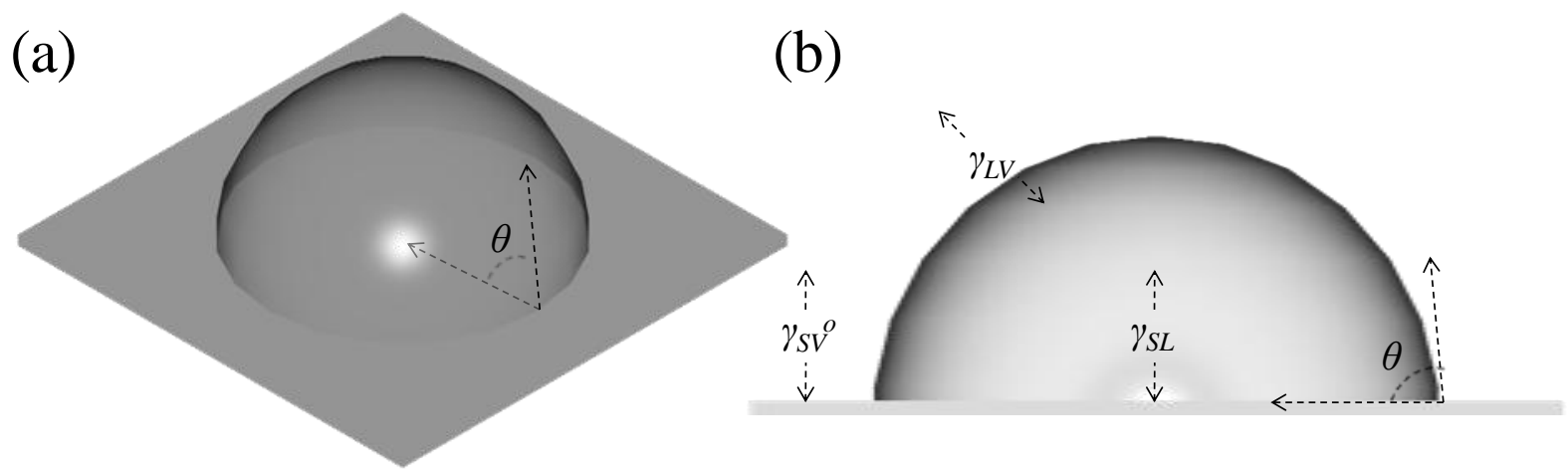

Figure 2.2 Illustration of sessile drop contact angle from (a) isometric view and (b) side view.

The energetics of wetting are known and can be used to derive a relation among phase surface tensions and the contact angle. Explicitly, the change in surface free energy, $\Delta G^{S}$, resulting in a fluid wetting additional surface area, $\Delta A$, of a solid substrate is given according to the following relation under the assumption that the surface is sufficiently smooth and chemically homogeneous (Adamson \& Gast, 1997).

$$
\Delta G^{s}=\Delta A\left(\gamma_{S L}-\gamma_{S V^{o}}\right)+\Delta A \gamma_{L V} \cos (\theta-\Delta \theta)
$$

In relation 2.31, the surface tension terms, $\gamma_{i}$, represent surface tension between solid-liquid $(S L)$, solid-vapor at a reference temperature and pressure $\left(S V^{\circ}\right)$ and liquid-vapor $(L V)$ phases 
as illustrated in figure2.2. At equilibrium considering the limit that $\Delta A \rightarrow 0$ and contact angle dynamics are negligible, the change in free energy per area gained will be null.

$$
\lim _{\Delta A \rightarrow 0} \frac{\Delta G^{s}}{\Delta A}=0
$$

As a consequence, an explicit relation between contact angle and surface tensions becomes apparent.

$$
\gamma_{L V} \cos \theta=\gamma_{S V^{o}}-\gamma_{S L}
$$

Relation 2.33 was originally developed circa 1805 by Young in a qualitative sense (Young, 1805) and later rigorously derived mathematically by Dupré (Dupré \& Dupré, 1869). Hence, relation 2.33 is commonly referred to as Young's equation. This quantity was also defined by Bartell, et al., as the tension of adhesion (Bartell \& Bartell, 1934).

$$
\Delta \mathrm{A}_{S L V}=\gamma_{L V} \cos \theta=\gamma_{S V^{o}}-\gamma_{S L}
$$

Young's equation is derived explicitly under the assumption that a surface is sufficiently smooth and homogenous in chemical composition. However, this is not always the case. Even for surfaces that appear smooth macroscopically may have roughness at the microscopic level affecting wettability. Accordingly, Wenzel, et al., suggested the modification of Young's equation to account for 'roughness' at the microscopic level where the correction factor, $r$, is the ratio of actual surface area to the surface area of a smooth surface with the same dimensions.

$$
\gamma_{L V} \cos \theta=r\left(\gamma_{S V^{o}}-\gamma_{S L}\right)
$$


For example, Wenzel notes that for a corrugated surface with an angle of corrugation of $45^{\circ}$, the correction factor to account for the surface roughness would be $r=\sqrt{2} A_{s} / A_{s}$, where $A_{s}$ is the surface area of a patch of sufficiently smooth surface (Wenzel, 1949).

A rich field of work exists attempting to understand the fundamental aspects of the formation of the contact angle at the atomic/molecular level. A great deal of experimental work has been done to study the variation in contact angle as a series of contact angles measurements are attained while the wetted area changes (Fowkes, 1963, Good, 1973, Neumann et al., 1974, Zisman, 1964). Furthermore, several empirical techniques exist explaining wetting phenomena based on these studies (Dann, 1970, Fowkes, 1963, Good \& Girifalco, 1960, Good, 1973). Although the empirical models work well for particular cases, Sullivan applies a more fundamental approach to describe the contact angle phenomena from Van der Waals interactions (Sullivan, 1981). Although Sullivan admits that the approach of using a 'hard-spheres with Kac tails' model is not necessarily the most accurate model for experimentally accessible contact angle data, the model provides the essential theoretical framework from which further study can extend Sullivan's results for more robust models. More recently, molecular dynamics has provided a means to probe the establishment of a three-phase contact line and associated contact angle through Langevin and Monte Carlo studies (Koplik et al., 1989, Nieminen et al., 1992). The results indicate that the assumption of no-slip does not appear to be adequate at the contact line of the three-phase interface.

For the current study, contact angle is measured through the sessile drop method in which a series of homologous droplets are placed on a solid surface and the angle is measured at solid-liquid interface via a Ramé Hart goniometer as the fluid droplet increases in volume. As will be demonstrated in chapter 3, the contact angle of both the hydrophilic 
and hydrophobic materials within the surface tension-confined microfluidic device have a drastic effect on the kinetics of capillary transport within the platforms (and incidentally the magnitude of pressure jump across the curved interface). Table 2.1 exhibits the contact angles attained for a handful of materials commonly used to fabricate STCM platforms that perform consistently. As indicated in table 2.1, even the moderately hydrophobic material poly(methyl methacrylate) can in fact function as a hydrophilic conduit since the contact angle is less than the $90^{\circ}$ limit where wetting no longer occurs spontaneously.

Table 2.1 Advancing Contact angle values for materials associated with functional STCM platforms as determined via the sessile drop method.

\begin{tabular}{rcc}
\hline Material & Purpose & Contact Angle \\
\hline Cellulose Acetate & Hydrophilic Conduit & $60.0(+/-0.3)$ \\
Poly(methyl methacrylate) & Hydrophilic Conduit & $73.0(+/-3.0)$ \\
Polystyrene & Hydrophobic Substrate & $79.0(+/-1.9)$ \\
Poly(vinyl chloride) & Hydrophobic Substrate & $82.4(+/-1.5)$ \\
\hline
\end{tabular}

Lastly, it is worth mentioning that contact angle measurements can exhibit hysteresis. Particularly with regard to the sessile drop method, the advancing contact angle can often be drastically different than the receding contact angle. In fact, the effect can be quite large. In some instances, a contact angle change of approximately $50^{\circ}$ has been observed for water on mineral surfaces (Adamson \& Gast, 1997). This effect is worth referencing; however, since the fluid filaments within the surface tension-confined platforms are always advancing when the device is implemented, this effect is not of great interest in the design and optimization of the polymer-based microfluidic platform. 


\subsection{Exploitation of Curvature for Capillary Flow}

Surface tension is of critical concern for surface tension-confined microfluidics - not only for fluid confinement but also as the driving force for transport actuation. Specifically, capillary driven flow occurs as a consequence of the general Laplace pressure drop $\Delta p=$ $2 \gamma H$. Essentially, spontaneous capillary flow occurs due to a pressure drop (or discontinuous jump) across the fluid interface as a consequence of surface tension and surface mean curvature, $H$. As a result, careful consideration must be given to the interface of a fluid undergoing capillary transport.

In the investigation of the transport aspects of a free surface, capillary-driven problem, there are two equally valid approaches for considering system pressure as long as one is careful setting up initial and boundary conditions. (1) It is possible to explicitly represent the pressure drop through mean curvature (see section 3.2.2) while considering gravity as the only force term (2) or one can leave system pressure undefined and consider the surface tension within the system as the driving force enabling the determination of the pressure as a variable as the problem evolves. For this study, the latter approach is selected since the implementation numerically is less abstract than the former technique.

The starting point of such an analysis is the utilization of the Navier-Stokes equations for incompressible Newtonian fluids in conjunction with the equation of continuity.

$$
\begin{array}{cr}
\rho\left[\frac{d \boldsymbol{u}}{d t}+\boldsymbol{u} \cdot \nabla \boldsymbol{u}\right]-\nabla \cdot\left[\mu\left(\nabla \boldsymbol{u}+\nabla \boldsymbol{u}^{\boldsymbol{T}}\right)\right]+\nabla p=\gamma\left(I-\left(n n^{T}\right)\right) \delta & 2.36 \\
\nabla \cdot \boldsymbol{u}=\mathbf{0} & 2.37
\end{array}
$$


In the equation system, $\rho$ is the liquid density, $\mu$ is the dynamic viscosity, $\boldsymbol{u}$ is the velocity vector, $p$ denotes the pressure, $\gamma$ is interfacial tension between two immiscible fluids (i.e. air and water/), $n$ is the normal along the liquid interface and $I$ denotes the identity matrix in three-dimensional space. The term $\delta$ is the three-space Dirac delta function ( $\delta=$ $\left.\left[\delta_{x}, \delta_{y}, \delta_{z}\right]^{\prime}\right)$ being non-zero in a particular direction only along the fluid interface. As a consequence of having to account for three phases with an advancing gas-liquid interface, presenting a discontinuity in density and viscosity, this problem is non-trivial and quite cumbersome.

The selected strategy to resolve numerical dilemmas representing the discontinuity associated with the advancing fluid interface is to utilize a level set method. Level set methods have recently become popular in fields such as two phase compressible and incompressible flow, flame propagation, and image processing, to name a few (Olsson \& Kreiss, 2005). Comprehensive description of level set methods can be found in references (Osher \& Fedkiw, 2002, Sethian, 1999) and specific applications to two-phase flow can be located elsewhere (Sussman et al., 1998, Sussman et al., 1994).

The drawback of level set methods in general is that conservation is not guaranteed. An artifact of this numerical method is that mass may be generated or consumed violating conservation principles. Recently, a conservative level set method has been established (Olsson \& Kreiss, 2005). By choosing an alternative level set method and advection scheme, mass is conserved. This is done utilizing a Heaviside step function (equal to 1 on one side of the interface and 0 on the other side, varying smoothly across the interface). The advection of the level set function is performed using a conservative scheme with an intermediate step requiring the shape and width of the profile across the interface to remain constant before 
proceeding to the next time step. This investigation implemented the reinitializing conservative level set method for the establishment the capillary dynamics realized within a surface tension-confined microfluidic device.

The reinitialized conservative level set method utilized to describe and convect the fluid interface works as follows. The 0.5 contour of the level set function $\varphi$ describes the fluid interface where $\varphi$ equals 0 in the bulk gas phase and $\varphi$ is equal to 1 in the bulk liquid phase while the function varies smoothly from 0 to 1 across the interface. Consequently the reinitialization scheme provides a means to track the advancing free surface. Furthermore, the normal to the interface is defined as follows (i.e. location of $\varphi=0)(A B, 2006)$.

$$
n=\frac{\nabla \varphi}{|\nabla \varphi|}
$$

The fluid velocity vector, $\boldsymbol{u}$, at the interface describes the advancement of the fluid front. The following equation defines the reinitialized convection of the level set function (AB, 2006).

$$
\frac{\partial \varphi}{\partial t}+\nabla \cdot(\varphi \boldsymbol{u})+\lambda\left[\nabla \cdot\left(\varphi(1-\varphi) \frac{\nabla \varphi}{|\nabla \varphi|}\right)-\varepsilon \nabla \cdot \nabla \varphi\right]=0
$$

With $\varepsilon$ being proportional to the interface thickness. It has been suggested that a structured mesh may be most efficient and to consequently allow $\varepsilon=2 h$ with $h$ as the minimum mesh resolution. The parameter $\lambda$ determines the amount of reinitialization. Furthermore, to smooth the viscosity and density discontinuity across the interface, the following relations are implemented.

$$
\begin{aligned}
& \rho=\rho_{g a s}+\left(\rho_{\text {liq }}-\rho_{g a s}\right) \varphi \\
& \mu=\mu_{g a s}+\left(\mu_{\text {liq }}-\mu_{g a s}\right) \varphi
\end{aligned}
$$

Lastly, to simplify the calculation of the surface tension force, allow $\delta=|\nabla \varphi|$. 
Coupling the Navier-Stokes system of partial differential equations with four dependent variables $\left(u_{x}, u_{y}, u_{z}\right.$, and $\left.p\right)$ along with the level set equation dependent upon $\varphi$ quickly becomes a very arduous task. Furthermore, in order to attain results, the system of non-linear coupled partial differential equations must be solved for a system with a density and viscosity discontinuity between multiple phases. Consequently, analytical solutions only occur in the most trivial cases. However, commercial software exists enabling such calculations numerically. For this study, COMSOL Multiphysics 3.3a is employed for the analysis.

The system is modeled as a two-dimensional rectangular duct with a height of 100 $\mu \mathrm{m}$ and a length of $1000 \mu \mathrm{m}$. The initial condition employed is $u_{x}=0$ at $t=0$ and $u_{y}=0$ throughout the problem-space. At the top and bottom substrate $(y=[0,100])$, a contact angle condition is specified as $\theta=\pi / 3$. However, in order to allow a contact angle condition numerically, a slip condition must also be specified. The slip length specified is $x_{\text {Slip }}=h / 2$ or a slip distance of one half of the minimum mesh size. Consequently, the integrity of the solution is maintained as maximum possible slip is below the minimum discernible feature size on the meshed geometry. The pressure at the initial interface location $(x=0$ for $y \in[0,100])$ is specified as the hydrostatic head pressure $p=\rho g y$. Figure 2.3 graphically denotes the system space for the numerical analysis.

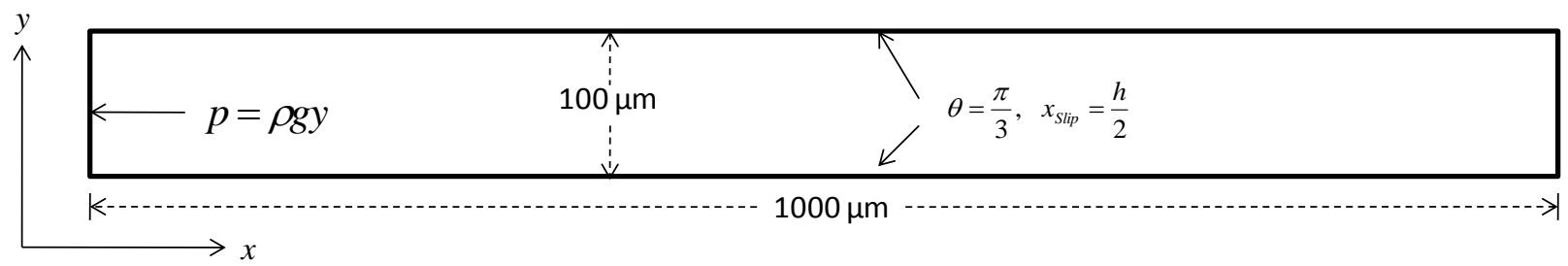

Figure 2.3 Graphical specification of system boundary conditions for conservative level set calculation of transient capillary transport. 
The problem is defined and implemented numerically COMSOL Multiphysics 3.3a. Predefined equation systems already exist in this software package for the coupling of the Navier-Stokes equations with the level set method for multiphase flow. The geometry is meshed with a structured grid to minimize the amount of computer resources required in the analysis. The built-in UMFPACK solver routine is utilized for a direct solution to the problem. Figure 2.4 demonstrates the results achieved via the numerical solution approach.

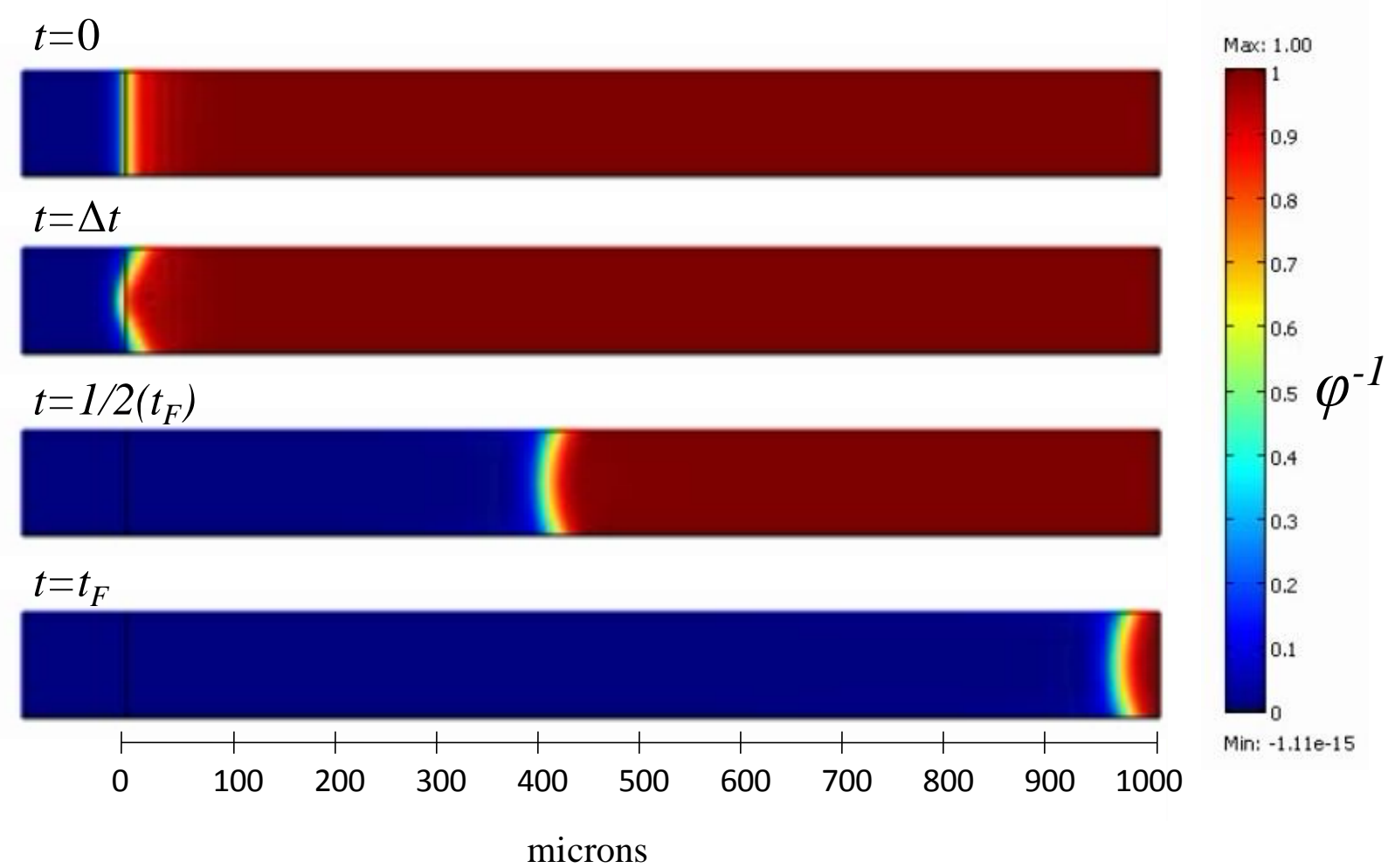

Figure 2.4 Conservative level set method results for two-phase capillary dynamics.

Figure 2.4 illustrates the inverse of the level set function, $\varphi^{-1}$ is 1 where $\varphi=0$ and $\varphi^{-1}$ is 0 where $\varphi=1$ (this is done strictly so that the blue phase represents the fluid component as blue is commonly associated with water - the red phase represents the gaseous component) for a total time requirement of 0.001 second with $\Delta t=2.5 e-5$. At initialization of the 
numerical scheme, the initial interface is flat and the pressure drop associated with curvature is zero and flow begins solely as a result of the hydrodynamic pressure. As the simulation progresses, curvature becomes evident and transport accelerates. Although it is difficult to discern from figure 2.4 , the curvature of the advancing front initially fluctuates and contact angle does not remain constant. The fluctuations begin to die out as the simulation progresses. At a time of $1 \times 10^{-3}$ seconds, the advancing front travels a total distance of approximately 960 microns. This result does not necessarily agree with relation 3.6 derived and discussed in chapter 3 which predicts the front should travel a distance of 1422 microns in $1 \times 10^{-3}$ seconds. However, equation 3.6 is derived under the assumption of negligible contact angle dynamics. In contrast, the static contact angle in the level set method is initially set as a constraint and enabled to evolve as the simulation proceeds. Due to lack of computer resources, the three-dimensional analog and greater time spans were not possible in this study. Furthermore, the desire to implement this calculation on more sophisticated machines is not necessary as relation 3.6 agrees rather well with experimental data and is drastically simpler to compute than a comprehensive solution to the Navier-Stokes equations coupled with the conservative level set method. Lastly, the employment of the numerical scheme utilizing slip conditions leaves the investigators skeptical of the ultimate validity of the simulation since the scientific community does not agree upon a common method for implementing slip velocities or slip lengths.

Although a comprehensive computational fluid dynamics model was not deemed to be imminently critical for device optimization, the ultimate curvature of the fluid interface is still very useful in the characterization of capillary dynamics. In fact, the curvature can be 
explicitly utilized to derive the Laplace capillary pressure jump. The following derivation follows the approach of J. A. Mann, Jr., closely (Mann Jr, 2002).

Considering a Monge surface of area $A_{o}$ in Cartesian coordinates, described by the mapping function $\xi(t, x, y)$, the change in free energy required to curve this surface with respect to a flat surface with a reference free energy, $\Omega_{0}$, at a constant composition and temperature is as follows.

$$
\Omega-\Omega_{0}=\int_{A_{o}}[p] \xi(t, x, y) \sqrt{a} d x d y+\int_{A_{o}} \gamma \sqrt{a} d x d y
$$

Where $[p]$ is the pressure drop across the interface and $a$ is a geometrical parameter for this derivation (rather than path width as in chapter 1).

$$
a=\sqrt{1+\left(\frac{\partial \xi}{\partial x}\right)^{2}+\left(\frac{\partial \xi}{\partial y}\right)^{2}}
$$

Considering that the surface $\xi(t, x, y)$ is arbitrary and the associated derivatives are small, the free energy function is simplified.

$$
\Omega-\Omega_{0}=\int_{A_{o}}[p] \xi d x d y+\int_{A_{o}} \gamma\left[1+\frac{1}{2}\left(\frac{\partial \xi}{\partial x}\right)^{2}+\frac{1}{2}\left(\frac{\partial \xi}{\partial y}\right)^{2}\right] d x d y
$$

Let $\delta$ be the variational operator and consider that $\xi(t, x, y)$ and the derivatives of $\xi(t, x, y)$ are equal to zero on the boundary, $\partial A_{o}$, over which the variation function is determined. Moreover, it is implicitly assumed that $\delta[p]=0$ and $\delta \gamma=0$.

$$
\delta \Omega=\int_{A_{o}}[p] \delta \xi d x d y+\int_{A_{o}} \gamma\left[\frac{\partial \xi}{\partial x} \frac{\partial \delta \xi}{\partial x}+\frac{\partial \xi}{\partial y} \frac{\partial \delta \xi}{\partial y}\right] d x d y
$$


Utilizing integration by parts on the second integral further simplifies the variation in free energy.

$$
\delta \Omega=\int_{A_{o}}[p] \delta \xi d x d y-\int_{A_{o}} \gamma\left[\frac{\partial^{2} \xi}{\partial x^{2}}+\frac{\partial^{2} \xi}{\partial y^{2}}\right] \delta \xi d x d y
$$

Because $A_{o}$ is arbitrary and $\delta \xi$ is small, but an otherwise arbitrary variation, an explicit pressure drop dependence becomes apparent.

$$
0=[p]-\gamma\left[\frac{\partial^{2} \xi}{\partial x^{2}}+\frac{\partial^{2} \xi}{\partial y^{2}}\right]
$$

Furthermore, in comparison with the Laplace pressure, $\Delta P=2 \gamma H$, the mean curvature of the Monge surface can be reconstructed.

$$
H=\frac{1}{2}\left[\frac{\partial^{2} \xi}{\partial x^{2}}+\frac{\partial^{2} \xi}{\partial y^{2}}\right]
$$

Consequently, the mean curvature is related to the concavity, or principal curvature (i.e. second derivatives of $\xi(t, x, y)$, of the surface. This result is explicitly utilized to calculate the pressure jump across the surface of an advancing fluid filament experienced within the surface tension-confined microfluidic device in chapter 3.

\subsection{Conclusions}

Surface tension and interface curvature are of profound importance to the design and function of surface tension-confined microfluidic platforms. As a result, a thorough understanding of these properties and the effect of outside influences on these properties is necessary for the development of suitable design criteria for chip optimization. 
Included within this analysis is a description of the influence of temperature on contact angle, and thereby, surface tension. This is done via utilizing a 2D Ising model and an expansion for surface tension based on contact angle. Through a rigorous analysis, it is possible to show that exact capillary wave theory and generalized capillary wave theory are in precise agreement in the characterization of angular momentum upon characterizing surface tension as an expansion dependent on contact angle.

Moreover, the extent of boundary versus bulk material is considered for the microfluidic system and the resulting effect on surface tension is derived. Ultimately, a change in surface tension begins to manifest itself as the bulk curvature of the system decreases to the characteristic size of the interface. This effect is more pronounced as the microfluidic regime decreases to the realm of nanofluidics.

In addition, thermal actuation of capillary transport is considered from a perspective of exploiting thermocapillary shear stress. Temperature changes and thermocapillary shear stress does not become an important factor in the implementation of microfluidic platforms in the ambient conditions. However, since current fabrication techniques parallel those utilized to fabricate micro-heaters, this effect is worth considering for the future design of microfluidic devices. Especially when applications require precise temperature control as in polymerase chain reaction, for example.

In addition, the dependence of surface tension on pressure is considered and corresponding Maxwell relations are derived through rigorous chemical thermodynamics. The result demonstrates that a change in pressure can affect phase partitioning. Again, the result suggests that the amount of bulk material to boundary material is of importance since the relationship is directly dependent upon interfacial width. 
Contact angle is discussed as a measure of energetic affinity between a fluid and substrate. This quantity is directly related to free energy change as a fluid wets a surface. Subsequently, Young's equation results under equilibrium conditions. Furthermore, the tension of adhesion is defined and a simple correction for rough surfaces is described.

Lastly, a discussion of modeling momentum transport for the free surface capillary flow is discussed along with the presentation of preliminary two-dimensional results. As the model is rather resource intensive to solve and does not lend too much insight into device optimization, three-dimensional results have not been thoroughly investigated. In lieu of the three-dimensional computational fluid dynamic results, a curvature associated pressure drop relationship is derived from first principles and will be explicitly utilized for the surface tension-confined microfluidic system in chapter 3. However, the framework for implementing a conservative level set method is established that can be utilized at a later time for the development of an exhaustive solution to momentum transport within the microfluidic platforms described herein.

\subsection{References}

AB, C. (2006). Inkjet Model, COMSOL Multiphysics, pp. 1-16.

Abraham, D. B. (1981). Physical Review Letters 47, 545-548.

Abraham, D. B. \& Reed, P. (1977). J. Phys. A 10.

Adamson, A. W. \& Gast, A. P. (1997). Physical Chemistry of Surfaces, 6-thed. WileyInterscience: New York.

Albert, R. K., Lakshminarayan, S., Hildebrandt, J., Kirk, W. \& Butler, J. (1979). J Clin Invest 63, 1015-1018.

Bachofen, H., Hildebrandt, J. \& Bachofen, M. (1970). Journal of Applied Physiology 29, 422-431. 
Bachofen, H., Schurch, S., Urbinelli, M. \& Weibel, E. R. (1987). Journal of Applied Physiology 62, 1878-1887.

Bartell, F. E. \& Bartell, L. S. (1934). Journal of the American Chemical Society 56, 22052210.

Buff, F. P., Lovett, R. A. \& Stillinger Jr, F. H. (1965). Physical Review Letters 15, 621-623.

Dann, J. R. (1970). J. Colloid Interface Sci 32, 302-331.

Darhuber, A. A. (2002). Journal of Applied Physics 91, 5686.

Darhuber, A. A., Davis, J. M., Troian, S. M. \& Reisner, W. W. (2003). Physics of Fluids 15, 1295-1304.

Darhuber, A. A. \& Troian, S. M. (2005). Annual Review of Fluid Mechanics 37, 425-455.

Darhuber, A. A., Valentino, J. P., Davis, J. M., Troian, S. M. \& Wagner, S. (2003). Applied Physics Letters 82, 657-659.

Defay, R. \& Prigogine, I. (1966). Surface Tension and Adsorption, translated by DH Everett, 1st ed. New York: Wiley-Interscience.

Delamarche, E., Bernard, A., Schmid, H., Bietsch, A., Michel, B. \& Biebuyck, H. (1998). J. Am. Chem. Soc 120, 500-508.

Dupré, A. M. \& Dupré, P. (1869). Théorie mécanique de la chaleur. Gauthier-Villars.

Fisher, M. P. A., Fisher, D. S. \& Weeks, J. D. (1982). Physical Review Letters 48, 368.

Fowkes, F. M. (1963). The Journal of Physical Chemistry 67, 2538-2541.

Gibbs, J. W. (1906). The Collected Works of J. Willard Gibbs. New Haven: Yale Univ. Press.

Good, R. J. \& Girifalco, L. A. (1960). The Journal of Physical Chemistry 64, 561-565.

Good, W. R. (1973). J. Colloid Interface Sci 44, 63.

Grigoriev, R. O. (2005). Physics of Fluids 17, 033601.

Herring, C. (1953). The Use of Classical Macroscopic Concepts in Surface Energy Problems, The Structure and Properties of Solid Surfaces, edited by R. Gomer \& C. S. Smith, p. 5. Chicago: University of Chicago Press. 
Jiao, Z., Nguyen, N. T., Huang, X. \& Ang, Y. Z. (2007). Microfluidics and Nanofluidics 3, 39-46.

Juncker, D., Schmid, H., Drechsler, U., Wolf, H., Wolf, M., Michel, B., de Rooij, N. \& Delamarche, E. (2002). Anal. Chem 74, 6139-6144.

Koplik, J., Banavar, J. R. \& Willemsen, J. F. (1989). Physics of Fluids A: Fluid Dynamics 1, 781.

Leamy, H. J., Gilmer, G. H. \& Jackson, K. A. (1975). Surface Physics of Materials, Vol. 1, p. 121. New York: Academic.

Lewis, G. N. \& Randall, M. (1923). Thermodynamics and the Free Energy of Chemical Substances. McGraw-Hill.

Mann Jr, J. A. (2002). Journal of dispersion science and technology 23, 199-207.

Neumann, A. W., Good, R. J., Hope, C. J. \& Sejpal, M. (1974). J. Colloid Interface Sci 49, 291-304.

Nieminen, J. A., Abraham, D. B., Karttunen, M. \& Kaski, K. (1992). Physical Review Letters 69, 124-127.

Olsson, E. \& Kreiss, G. (2005). Journal of Computational Physics 210, 225-246.

Osher, S. \& Fedkiw, R. P. (2002). Level Set Methods and Dynamic Implicit Surfaces. Springer.

Sethian, J. A. (1999). Level set methods and fast marching methods. Cambridge University Press Cambridge.

Sullivan, D. E. (1981). The Journal of Chemical Physics 74, 2604.

Sussman, M., Fatemi, E., Smereka, P. \& Osher, S. (1998). Computers \& Fluids 27, 663-680.

Sussman, M., Smereka, P. \& Osher, S. (1994). Journal of Computational Physics 114, 146159.

Tolman, R. C. (2004). Journal of Chemical Physics 17, 333.

Turkevich, L. A. \& Mann, J. A. (1990). Langmuir 6, 445-456.

Volkoviski, V. (1935). CR Acad. Sci (Paris), Compt. Rend 200, 1285-1287.

Wenzel, R. N. (1949). The Journal of Physical Chemistry 53, 1466-1467. 
Young, T. (1805). Peacock and J. Murray, London 418.

Zisman, W. A. (1964). Adv. Chem. Ser 43. 


\section{Chapter 3: Design and Fabrication of Autonomous}

\section{Microfluidic Platforms}

\subsection{Literature Review}

Microfluidic and 'lab on a chip' technology is speculated to revolutionize the way in which chemical and biological analyses are conducted. Such devices promise the capacity to manipulate micro-volumes of fluid in order to maximize throughput with greater degrees of parallelization while minimizing the amount of expensive and/or invasive reagent. To date, several novel microfluidic systems have been developed demonstrating the automation of clinical techniques such as the enzyme-linked immunosorbent assay (Eteshola \& Balberg, 2004, Eteshola \& Leckband, 2001, Sato et al., 2004) and DNA analyses (Burns, 1998, Khandurina et al., 2000, Lagally et al., 2000, Mastrangelo et al., 1998), as well as novel applications for fuel cell development and optimization (Choban et al., 2004, Jayashree et al., 2005, Mitrovski et al., 2004).

However, the anticipated impact of microfluidic technology has yet to have materialized for a combination of reasons. For example, fabrication methods can be cost ineffective and time intensive. Furthermore, most platform manufacturing techniques require batch processing rather than continuous production. In addition, platform implementation may require auxiliary hardware such as thermal elements, pumping systems and equipment for optical detection further complicating the platform design rendering the device susceptible to component failure and overall device malfunction. Consequently, before microfluidic technology can be deployed on a wide-scale, it is essential to develop 
manufacturing methodology with limited rigor as well as designing micro-devices capable of autonomous fluid manipulation.

An alternative paradigm to microfluidic processing involves patterning abrupt discontinuities in surface energy upon a substrate. Consequently, high surface tension fluids can be confined upon regions of higher surface energy in spite of the lack of physical barriers. Patterned substrates are aligned and fixed to each other with intermediate spacers (figure 3.1). Following introduction of a high surface tension fluid, spontaneous capillary wicking occurs as a result of a pressure drop associated with a curved surface. Moreover, confinement is achieved at the three-phase hydrophobic interface via fluid-substrate energetics. This technique was first employed for capillary electrophoresis (Oh, 1999). Subsequently, other groups have further investigated methods to fabricate similar devices via sol gel processing (Lam et al., 2002), monolayer development with octadecyltrichlorosilane by multi-stream laminar flow or photolithography (Bouaidat et al., 2005, Suk \& Cho, 2007, Zhao et al., 2001, 2002) and even simple methods such as painting hydrophobic barriers (Nag et al., 2005). Recently, a technique has been employed using a microplasma jet to create hydrophilic pathways on chemically-modified hydrophobic glass resulting in parallelplate millifluidic systems (West et al., 2007). These techniques demonstrate the efficacy of employing two-dimensional surface energy discontinuities to support autonomous microfluidic transport. 


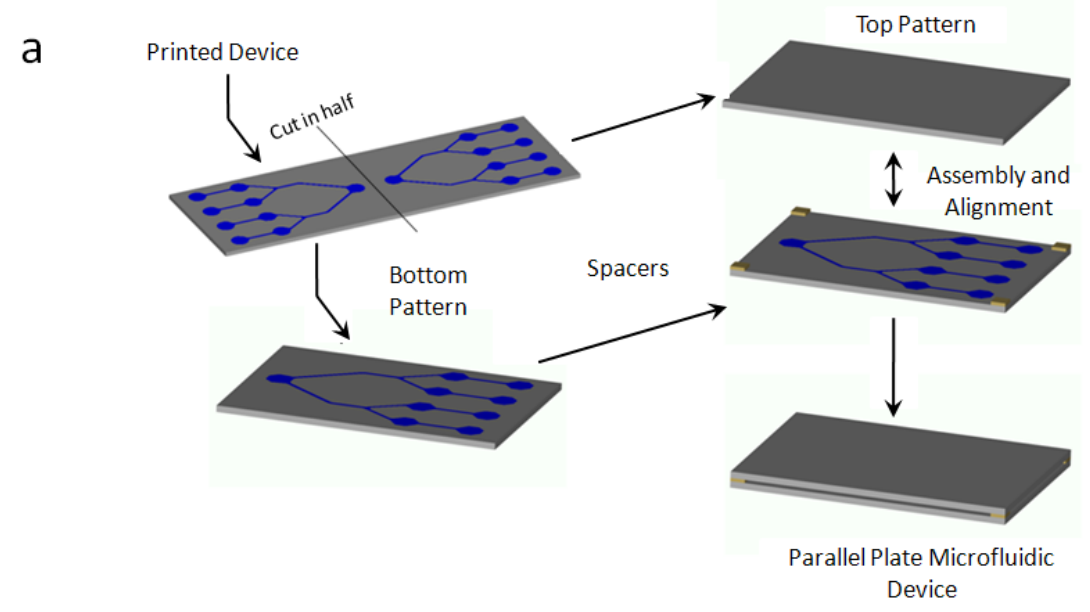

b

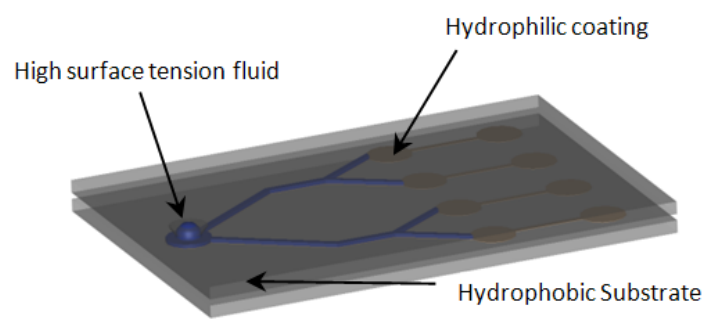

Figure 3.1 a Configuration of surface tension-confined microfluidic platform from printed substrate to parallel-plate microfluidic device. b Implementation of surface tension confined microfluidic device.

Recently, efforts have been made to exploit microfabrication technology in order to develop manufacturing methodology conducive to mass production. For example, Watanabe has produced autonomous microfluidic platforms via inkjet printing of highly water soluble paths upon a hydrophobic substrate material capable of directing surface flow (Watanabe, 2007). Described herein are alternative fabrication methods involving the thick film printing of cellulose acetate, or pen plotting of silica sol gel, upon a polyvinyl chloride (PVC) substrate material. The one-step patterning of these materials develops adequate disparity in surface energy to facilitate spontaneous fluid transport and excellent confinement of aqueous solutions upon the hydrophilic paths. Furthermore, silica sol gel and cellulose acetate are attractive materials as they have already demonstrated compatibility with biological 
components (Goodwin et al., 1995, Marengo-Rowe, 1965, Meera Khan, 1971) as opposed to other common materials utilized in the development of microfluidic platforms such as polydimethylsiloxane (Butler, Lue et al., 1997, Butler, Navarro et al., 1997). Moreover, thick film printing is an established process thoroughly understood and documented having been around for nearly 40 years (Holmes, 1976, White \& Turner, 1997). Consequently, optimization is straightforward minimizing the rigor associated with implementing massproduction processing. Additionally, the choice of commodity polymeric materials such as PVC and cellulose acetate assist in mitigating the device manufacturing cost. Furthermore, the mechanical properties of the PVC substrate material are more advantageous for on-site application than brittle materials such as silicon or glass. Ultimately, the author envisions the one-step production scheme amenable to mass-production implemented continuously.

\subsection{Hydrophilic Patterning of Hydrophobic Substrates}

Generating discontinuities in surface energy, either through surface patterning or selective surface chemistry, facilitates the control of wettability of fluids upon that surface. Surface tension-confined microfluidics (STCM) is the exploitation of this phenomenon to encourage fluid transport through capillary interactions upon a surface-modified platform. Consequently, the need for extraneous pumping components is eliminated. These techniques demonstrate a great capacity to automate fluid transport providing alternative manufacturing methods for microfluidic platform development. Described below are fabrication processes that can be utilized for generating disparities in surface energy. Subsequently, if patterned substrates are configured cleverly, this technique can be used for the development of fully autonomous microfluidic platforms. 
The consideration of system energetics lends insight into geometrical criteria required for spontaneous capillary flow. Figure 3.2, produced via minimizing a free energy through a gradient descent method via the Surface Evolver software package (Brakke, 1992), depicts the expected geometry of a fluid filament undergoing capillary transport in a surface tensionconfined platform. Specifically, for the geometry and orientation demonstrated in figure 3.2, the free energy, $G$, associated with a fluid of surface tension $\gamma$ wetting a substrate for a length $l$ dependent upon a gap height $h$ and path width $w$ of a material characterized by an advancing contact angle $\theta$ as follows (Adamson \& Gast, 1997, Lam et al., 2002).

$$
\frac{d G}{d l}=\gamma(h-w \cos \theta)
$$

Consequently, in order to satisfy a negative free energy required for the surface tensionconfined device to support spontaneous flow, the gap height must be less than the product of hydrophilic path width and the cosine of the advancing contact angle (i.e. $h<w \cos \theta$ ).

a

b
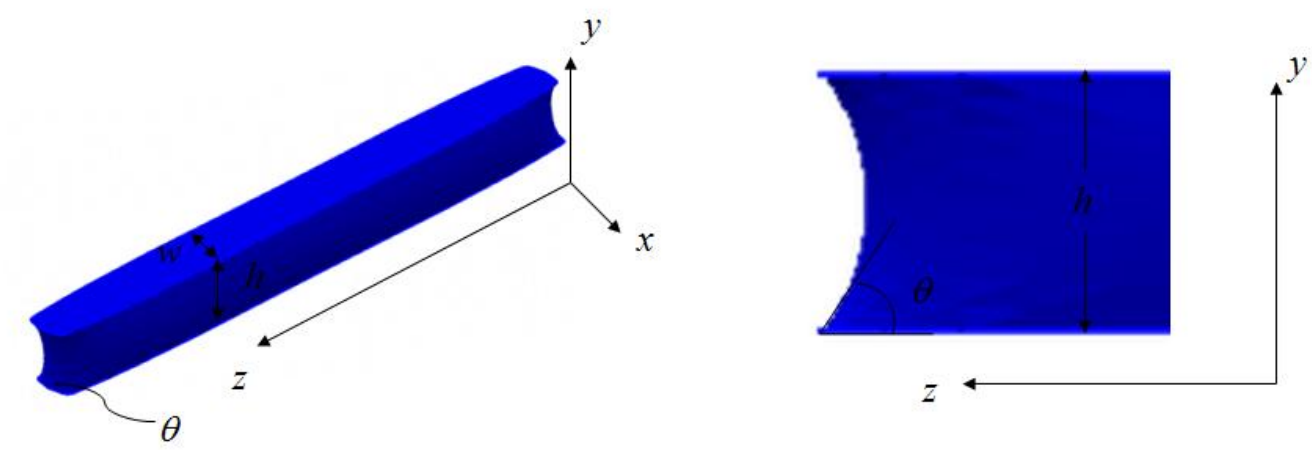

Figure 3.2 Geometry of a high surface tension fluid progressing across a hydrophilic path in a surface tension-confined microfluidic device. a Isometric view and $\mathbf{b}$ side view. 
In addition, it is apparent that materials with contact angle greater than or equal to $90^{\circ}$ are incapable of supporting capillary transport. One can also arrive at the same result based on arguments of achieving a negative pressure drop (Zhao et al., 2001, 2002).

\subsubsection{Sol-Gel Processing and Pen Plotting}

The initial approach to fabricating STCM devices entailed reproducing the results attained by Lam et al. at Virginia Commonwealth University (Lam et al., 2002). In the previous study, Lam et al. patterned hydrophobic substrate materials with silica sol gel utilizing an HP7475A pen plotter. The advantage of this approach is patterning a hydrophilic material upon a hydrophobic substrate results in a slightly raised path material minimizing any contact between a biological component and hydrophobic material. This advantage mitigates significant and irreversible adsorption and denaturation of biological entities that commonly occur through hydrophobic interactions (Butler, Lue et al., 1997, Butler, Navarro et al., 1997). Furthermore, from the onset of the investigation, it was suggested that the hydrophobic substrate, which also functions as the platform support, must have adequate mechanical properties to withstand punishment that may occur in adverse environments for instances in which field application is required. Several initial candidate hydrophobic materials include, but are not limited to, include polystyrene, polyethylene, polypropylene, polyvinyl chloride, polycarbonate and poly(methyl methacrylate). Furthermore, for at least the top substrate material, an appropriate material must be optically transparent for visual or fluorometric/colorimetric detection of response.

The hydrophilic wicking material chosen at the onset of the investigation is based on preceding research and slated to be silica sol gel. The sol gel process, facilitated by a number 
of alternative reaction pathways, is essentially a chemical route to prepare glass (Gill \& Ballesteros, 2000). This material is demonstrated as successful for a surface tension-confined microfluidic approach and can be patterned with minimal rigor through techniques such as pen plotting. Other advantages of the sol gel chemistry is that it can be extended to incorporate biological components through lattice entrapment techniques.

Lattice entrapment techniques confine proteins within porous matrices. This microencapsulation technique does not create covalent bonds with the protein enabling a wide distribution of orientations upon a given substrate ensuring the availability of active sites (Rupcich \& Brennan, 2003). Consequently, the porous morphology and microstructured surface area of the lattice structures allows for high biomolecule loading with a large extent of the bioactivity remaining (Nadzhafova et al., 2004). Moreover, biocompatible precursors and additives can be added to maintain optimal levels of activity (Rupcich \& Brennan, 2003).

Initial efforts preliminarily established the efficacy of sol gel as a wicking agent. Early experiments included attempting to swab or electrospray sol gel solution upon a masked substrate material. Sol gel is prepared through precursors such as tetraethyl orthosilicate in water with an acid catalyst or a variety of polyalkoxysiloxanes in isopropanol with the catalyst dibutyltin diacetate.

The optimized protocol for silica sol gel production using an alkoxysiloxane route is as follows. A polyalkoxysiloxane precursor is utilized as the starting material (polydiethoxysiloxane $40 \% \mathrm{SiO}_{2}$ equivalent, polydiethoxysiloxane $50 \% \mathrm{SiO}_{2}$ equivalent and polydimethoxysiloxane $57 \% \mathrm{SiO}_{2}$ equivalent; Gelest, Inc.). A 9\% (w/w) solution of the precursor is made in isopropanol. The catalyst, dibutyltin diacetate (Sigma Aldrich) is added 
to the precursor solution at a concentration of $2 \mu 1$ per gram of total solution which acts as a Lewis acid. The solution is then applied to a sufficiently clean hydrophobic substrate material via electrospraying, 'painting' or pen plotting. Subsequently, the patterned substrate is then incubated in a humid environment at $40{ }^{\circ} \mathrm{C}$ for approximately 1 hour. Following incubation, the film is exposed to $1.0 \mathrm{M} \mathrm{HCl}$ for 60 seconds resulting in full hydrolysis of the glass-like material and syneresis of the precursor to a three-dimensional $\mathrm{SiO}_{2}$ matrix. The higher the $\mathrm{SiO}_{2}$ equivalent, the less syneresis occurs resulting in paths with better integrity. The patterned substrate is then washed with a copious amount of water during which preferential wetting is apparent. Figure 3.3 demonstrates the reaction process associated with each step of the polymerization process. Figure 3.4 indicates initial success with the sol gel material using the preliminary masking techniques for patterning.

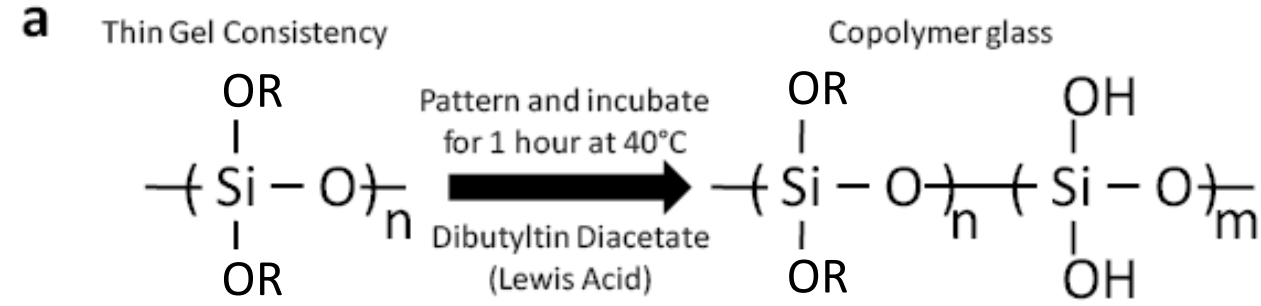

b

Copolymer glass

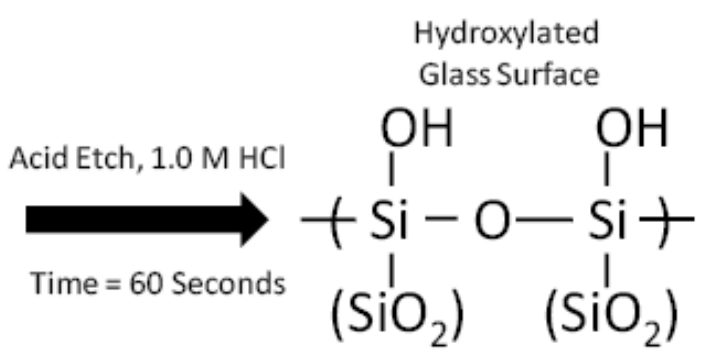

Bulk Glass

$$
\mathrm{R}=\mathrm{CH}_{3} \text { or } \mathrm{CH}_{2} \mathrm{CH}_{3}
$$

Figure 3.3 Reaction mechanism of sol gel polymerization via the polyalkoxysiloxane route. a Polymerization of polyalkoxysiloxane oligomer to a copolymer glass b Hydrolysis and syneresis of copolymer glass to $\mathrm{SiO}_{2}$ glass matrix. 


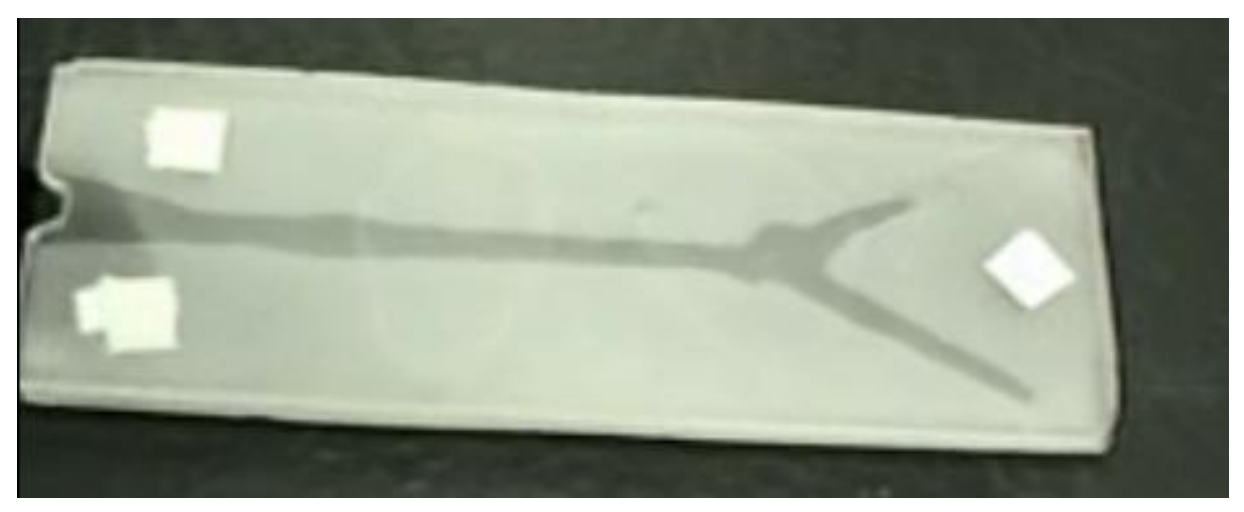

Figure 3.4 Device fabricated via sol gel processing upon a roughened polystyrene surface.

Following preliminary experiments via masking off a substrate material with tape, investigation was conducted to automate the process with a HP7475A pen plotter interfacing with the AutoCAD 2005 software package. Figure 3.5 demonstrates several patterns developed using the pen plotter as a patterning device. Although the pen plotter could successfully pattern a substrate, results utilizing this technique were largely inconsistent with a very poor rate of success.
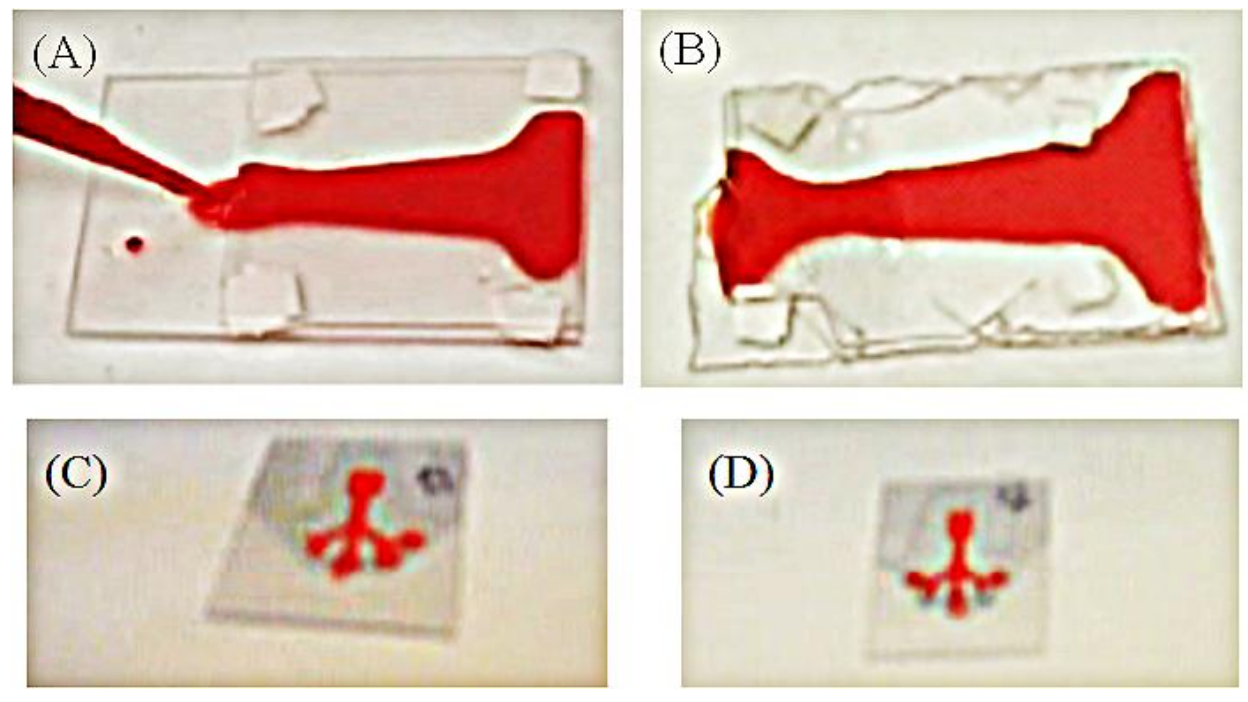

Figure 3.5 Devices made with pen plotting sol gel on (A) polypropylene $\sim 200 \mu 1$, (B) polypropylene $\sim 200 \mu \mathrm{l}$ and $(\mathbf{C}, \mathbf{D})$ polyvinyl chloride $\sim 25 \mu \mathrm{l}$. 
Because of the inconsistencies associated with pen plotting, alternative patterning methods are of primary concern for the development of reproducible STCM platforms. Due to importance on the criteria of robustness, a well-understood patterning method is critically important. Consequently, thick film printing could result in a method for reproducibly manufacturing microfluidic platforms as this technology has been around over 40 years and has been utilized to produce semiconductor devices, monolithic interconnects, printed circuit boards and other discrete devices (White \& Turner, 1997). The thick film technique also affords the opportunity to integrate non-fluidic components into the devices as well if such a need exists for future studies (e.g. microheaters for thermocapillary induction, electrodes for electro-osmotic pumping, electronics for electrochemical detection techniques).

\subsubsection{Thick Film Printing of Hydrophilic Polymers}

\section{Screen Development}

Thick film printing screens are made through a one-time photolithographic technique. Patterns are developed with computer-aided drafting software and transferred to transparency films through a Dell Laser Printer 1700 for the development of a photomask. The photomask is utilized on emulsion film (Five Star, Autotype) via exposure to UV in dark room conditions for three minutes and forty-five seconds. The emulsion film is subsequently developed in $1.5 \%(\mathrm{v} / \mathrm{v})$ hydrogen peroxide solution for one minute. After development, the film is sprayed with copious amounts of warm tap water $\left(\sim 50^{\circ} \mathrm{C}\right)$. At this point, the pattern should be evident in the film. The developed film is now placed in cool distilled water for 
thirty seconds $\left(\sim 20^{\circ} \mathrm{C}\right)$. The film is transferred to a 325 mesh stainless steel screen with 0.0011 inch wire diameter via applying pressure with a roller. After subsequent drying time $\left(\sim 1\right.$ hour at $\left.35^{\circ} \mathrm{C}\right)$, the plastic coating is peeled from the emulsion film resulting in a patterned screen. The entire process takes approximately 1.5 hours and the resulting screen can be used indefinitely if proper maintenance is conducted. Following screen implementation, the screen is cleaned with acetone resulting in no degradation of the photoresist film over the multiple cleaning cycles commenced during this investigation. As a standard office laser printer is used for this process, the minimum attainable resolution that can be achieved in the photoresist film is approximately $225.8 \pm 37.0 \mu \mathrm{m}$ as determined through optical microscopy of the photoresist film.

\section{Substrate Patterning}

Rinzl vinyl microscope slides (Fisher Scientific) reported as polyvinyl chloride (PVC) by the manufacturer are cleaned with ethanol prior to patterning. Although this material is reported as PVC by the manufacturer, elemental analysis indicates that $4.19 \%(\mathrm{w} / \mathrm{w})$ of the material is an element other than hydrogen, chlorine or carbon suggesting that the material is not pure PVC. Furthermore, the presence of a carbonyl band at $1728 \mathrm{~cm}^{-1}$ demonstrated through Fourier-transform infrared spectroscopy and the flexibility of the material suggests to us that the material is likely a vinyl chloride-vinyl acetate copolymer. Great care must be taken to keep cleaned vinyl slides away from surface contamination as minute amounts of contaminants can drastically affect wettability due to the surface area to volume ratios experienced in microfluidic platforms (Squires \& Quake, 2005). Thick film ink is prepared from cellulose acetate (39.7\% acetylated, Mn 30,000, Sigma Aldrich) dissolved in 
tetraethylene glycol dimethyl ether (Sigma Aldrich), chosen for an inherently low volatility, at various concentrations.

The custom screen is employed for patterning in a MPM TF-100 thick film printer at the Case Electronics Design Center. A small amount of ink is placed directly on the screen in front of the open pattern. The thick film printer squeegee mechanism is actuated and the cellulose acetate ink is pressed through the open pattern in the screen onto the vinyl microscope slide. It is critical that the solvent have sufficiently low volatility as not to evaporate when the ink is in the screen causing the mesh to become blocked by dry polymer. Patterned substrates are placed in vacuum conditions at ambient temperature to cure overnight. After the solvent completely evaporates, the patterned substrates are once again cleaned with ethanol and dried in ambient conditions prior to assembly and implementation. Following the cleansing procedure, preferential wetting is immediately apparent. The adhesion of the cellulose acetate to the PVC slides is rather good. Furthermore, as the PVC and patterned cellulose acetate materials are flexible, bending of a patterned substrate does not delaminate the hydrophilic film.

A comprehensive investigation on printing resolution was conducted for inks of varying polymer concentration $(8 \%, 12 \%, 15 \%$ and $18 \%, \mathrm{w} / \mathrm{w})$. Assorted path widths were printed from nominal CAD values ranging from $300 \mu \mathrm{m}$ to $1300 \mu \mathrm{m}$ as summarized by table 3.1. A total of 10 patterns were printed per concentration along with 5 measurements taken randomly along a path for the determination of path width. As indicated by table 3.1 and graphically by figure 3.6 , the $8 \%$ ink resulted in paths generally below the nominal CAD value as a consequence of the de-wetting of the low viscosity ink. In contrast, the $12 \%$ ink tended to be thick enough to flow slightly upon printing but was not capable of de-wetting 
and forming beaded droplets as in the case of the $8 \%$ ink. Both the $15 \%$ and $18 \%$ inks were found to be relatively close to the CAD design. Figure 3.6 graphically summarizes the results for this study.

Table 3.1 Thick film-printed pattern width as a function of ink concentration and path width.

\begin{tabular}{|c|c|c|c|c|c|c|}
\hline $\begin{array}{c}\text { Nominal } \\
\text { Width } \\
(\mu \mathrm{m})\end{array}$ & $\begin{array}{c}\text { Photomask } \\
\text { Width }(\mu \mathrm{m})\end{array}$ & $\begin{array}{c}\text { Screen } \\
\text { Width }(\mu \mathrm{m})\end{array}$ & $\begin{array}{c}8 \% \text { Width } \\
(\mu \mathrm{m})\end{array}$ & $\begin{array}{c}12 \% \text { Width } \\
(\mu \mathrm{m})\end{array}$ & $\begin{array}{c}15 \% \text { Width } \\
(\mu \mathrm{m})\end{array}$ & $\begin{array}{c}18 \% \text { Width } \\
(\mu \mathrm{m})\end{array}$ \\
\hline 300 & $313.2 \pm 8.4$ & $225.8 \pm 37.0$ & $242.1 \pm 43.6$ & $329.2 \pm 14.8$ & $321.7 \pm 12.6$ & $313.3 \pm 17.9$ \\
\hline 550 & $522.1 \pm 8.2$ & $458.4 \pm 37.4$ & $381.5 \pm 60.7$ & $696.8 \pm 38.7$ & $546.3 \pm 14.8$ & $583.8 \pm 26.1$ \\
\hline 800 & $804.2 \pm 10.6$ & $727.16 \pm 23.7$ & $419.5 \pm 50.5$ & $851.3 \pm 18.9$ & $799.4 \pm 26.2$ & $830.9 \pm 35.5$ \\
\hline 1050 & $1068.7 \pm 12.1$ & $998.2 \pm 62.0$ & 1036.788 .3 & $1122.9 \pm 45.2$ & $1031.9 \pm 17.0$ & $1146.9 \pm 9.3$ \\
\hline 1300 & $1326.6 \pm 8.3$ & $1293.7 \pm 29.4$ & $1247.4 \pm 43.3$ & $1326.3 \pm 41.6$ & $1318.6 \pm 22.1$ & $1352.1 \pm 16.0$ \\
\hline
\end{tabular}

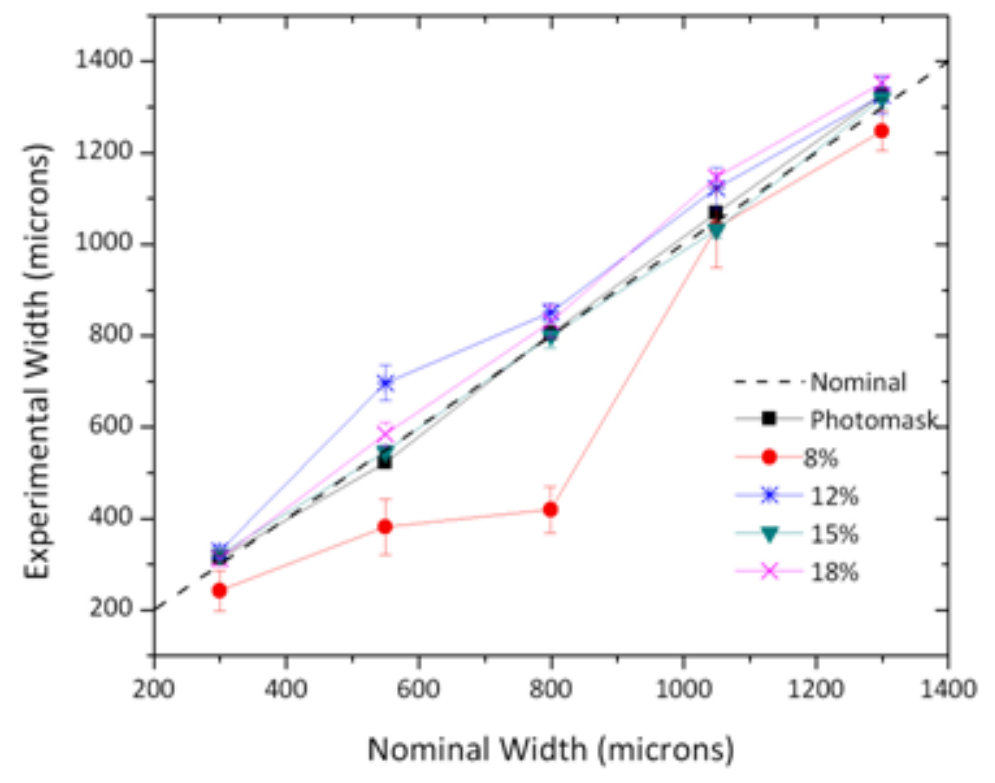

Figure 3.6 Graphical summary of thick-film printed paths versus polymer concentration. 


\section{Platform Assembly}

Holes are drilled for fluid introduction at strategically placed locations on the patterned substrates. Opposing patterned substrates are visually aligned and brought within close proximity utilizing $116.4 \pm 7.6 \mu \mathrm{m}$ thick 9576 double-sided polypropylene film tape $(3 \mathrm{M})$ as determined utilizing a series of micrometer measurements on several assembled devices. The devices of $229.0 \pm 13.9 \mu \mathrm{m}$ were assembled by using a double layer of polypropylene tape. Following alignment, devices are ready for the introduction of an aqueous fluid resulting in autonomous capillary pumping and excellent confinement within intricate geometry in spite of the lack of physical barriers. Care must be taken to keep microfluidic platforms away from airborne contaminants. We have found that storage in ambience results in loss of reproducible function within one week. In contrast, storage in a sealed environment seems to inhibit surface contamination leading to the preservation of adequate functionality. However, function can be restored to contaminated devices at any time by simply conducting an additional ethanol cleaning procedure.

\section{Contact Angle Analysis}

The advancing contact angles of the materials were attained utilizing a Ramé-Hart goniometer. A series of five measurements were attained to calculate an average contact angle. The PVC material exhibited a contact angle of $82.4^{\circ} \pm 1.5^{\circ}$ while the cellulose acetate contact angle was measured as $60.0^{\circ} \pm 0.3^{\circ}$. Both materials have contact angles less than $\pi / 2$ indicating that the fluid is capable of transit across each material via capillarity. However, since there is substantially less resistance for the fluid to progress in the direction of the hydrophilic cellulose acetate, directed flow can be achieved in the microfluidic platform. 
Attaining Distance versus Time Data

Transparent rulers are fixed to the operational region of the microfluidic platforms. Distilled water is introduced to the platforms and capillary dynamics are recorded on a digital camcorder. The resulting digital media files are converted to mpeg format and analyzed frame by frame with the VirtualDub software package. The resulting data is utilized to construct position versus time curves.

\section{Demonstration of Function}

Following the fabrication protocols previously outlined results in microfluidic platforms capable of supporting autonomous fluid transport. Furthermore, since screen processing is relatively simple, a great deal of versatility is attained through the thick film printing process. Figure 3.7 demonstrates several patterns that have been successfully developed through the thick film printing process along with a variety of solutions utilized within the platforms. 


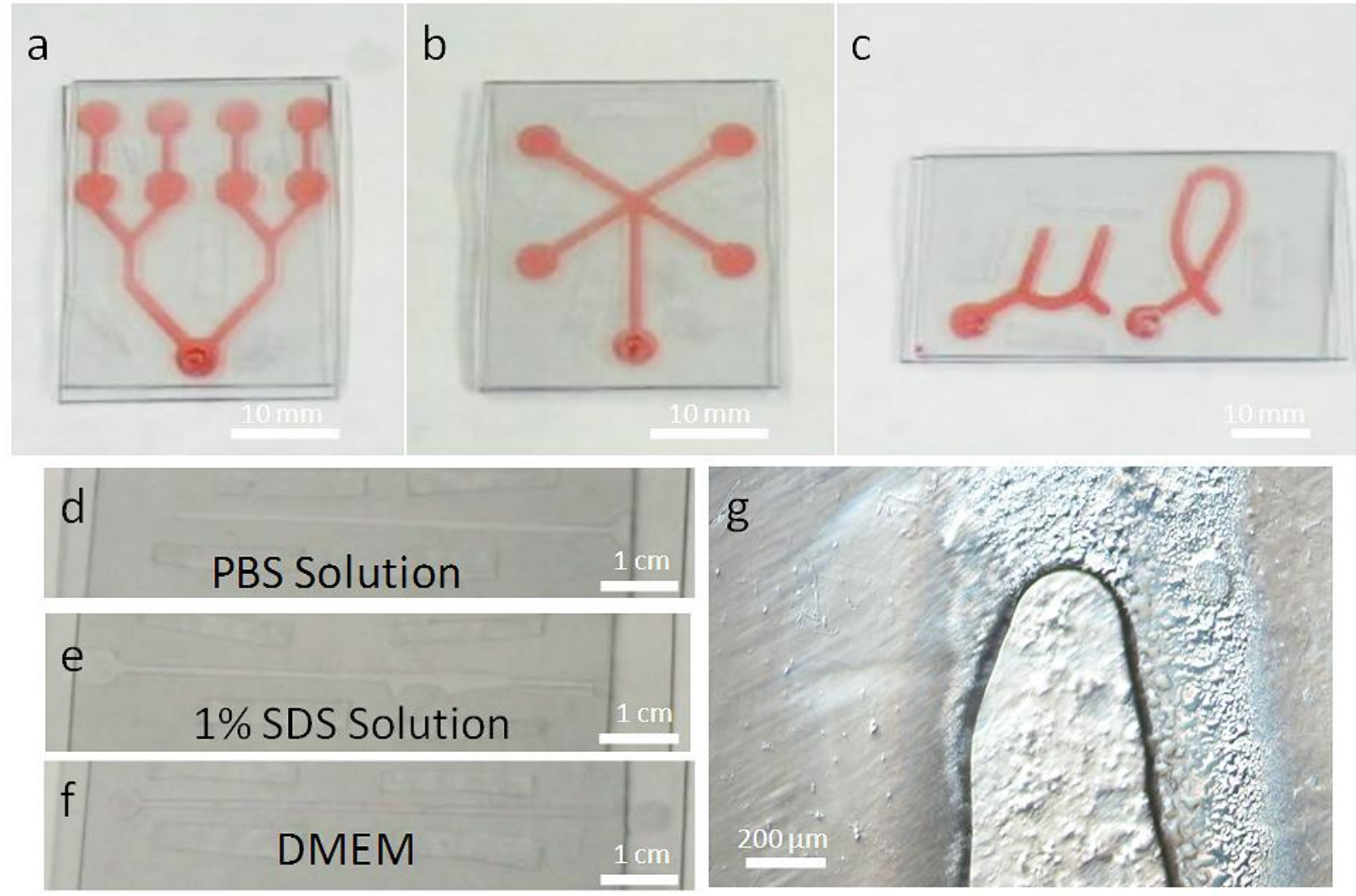

Figure 3.7 Demonstration of autonomous surfaced-directed microfluidic platforms. a Diverging pattern - $19 \mu \mathrm{l} \mathbf{b}$ Four-well design $-15 \mu \mathrm{l}$ c 'Microliter' $-15 \mu \mathrm{l} \mathbf{d}$ Device implemented with PBS, e SDS, and $\mathbf{f}$ DMEM g Device with minimum attainable resolution.

As demonstrated by figure 3.7, the amount of fluid required to implement the surfacetension confined device is in all cases below 20 microliters of fluid. Furthermore, when implementing the device of minimum resolution (313.3 \pm 17.9 micron path width, $229.0 \pm 13.9$ $\mu \mathrm{m}$ gap height), the amount of fluid required is reduced to approximately 2 microliters in order to transport fluid across a $6.0 \mathrm{~cm}$ virtual channel. In addition to dyed-distilled water, the microfluidic platform were also implemented with biologically relevant solutions with drastically different surface tension values than pure water such as phosphate buffered saline (PBS), 1\% (w/w) aqueous solution of the surfactant sodium dodecyl sulfate (SDS) and Dulbecco's Modified Eagle Medium (DMEM). The DMEM and PBS solutions maintain 
rather good confinement across the microfluidic channel. In contrast, directed flow can be achieved with $1 \%$ SDS; however, fluid does not maintain excellent confinement across the length of the hydrophilic path.

Furthermore, pattern integrity was investigated as a function of ink viscosity. Viscosities on a weight per weight basis were investigated at concentrations of $8 \%, 12 \%$, $15 \%$ and $18 \%$ cellulose acetate in tetraethylene glycol dimethyl ether. The $8 \%$ solution results in a path of very poor integrity leaving the bare substrate exposed as the hydrophilic ink began to de-wet and 'bead' on the hydrophobic substrate material. Remarkably, the devices patterned with $8 \%$ hydrophilic ink were still capable of supporting autonomous fluid transport although reproducibility is an issue. As ink viscosity increases, pattern integrity becomes substantially better as 'beading' becomes inhibited through viscous forces. The 18\% solution seems to be the practical limit where cellulose acetate will no longer dissolve into the solvent without extensive heating and agitation. Furthermore, the film thickness of the printed paths were determined through imaging cellulose acetate that was encouraged to delaminate from the PVC substrate with a razor blade. Figure 3.8 illustrates a typical image attained via scanning electron microscopy demonstrating that film thickness is on the order of approximately one micron. Higher magnification images were unattainable as a consequence of the low melting point of cellulose acetate and heat generated through further focusing of the electron beam. 


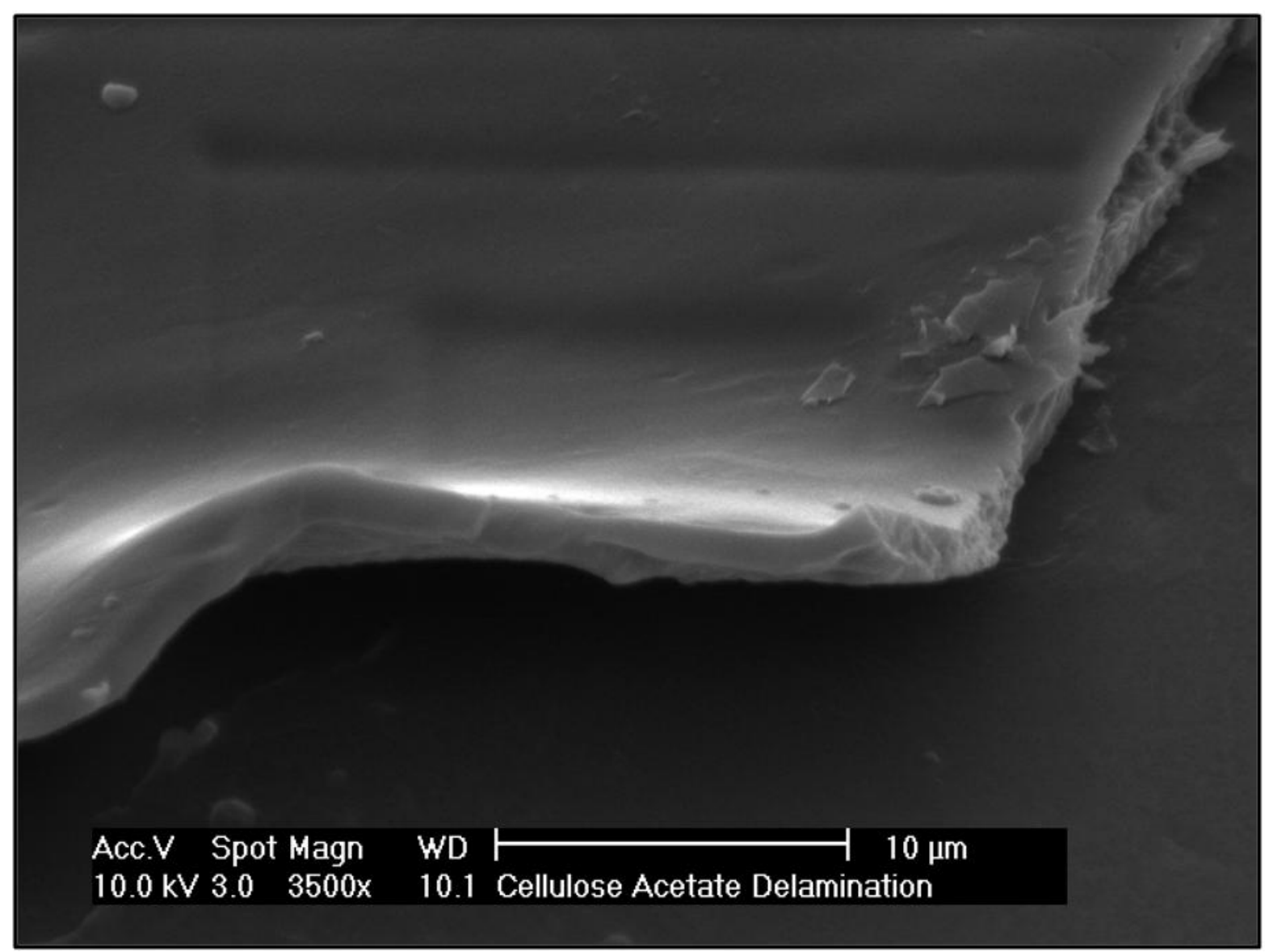

Figure 3.8 Scanning electron micrograph indicating relative thickness of a thick film-printed cellulose acetate path encouraged to lift from the substrate material with the tip of a razor blade.

Theoretical Aspects and Characterization

Although a consistent and versatile fabrication procedure exists for the development of surface-directed microfluidic devices, a theoretical model is very beneficial for a priori device optimization and intelligent device design with respect to flowrate and residence in support of on-chip reaction processes. The system is defined as a having a symmetrically centered axis with $-w / 2 \leq x \leq w / 2$ and $-h / 2 \leq y \leq h / 2$. For the position $x=0$, end effects are neglected since $h \ll w$. Consequently, the fluid transport is idealized to the canonical flow of 
an incompressible fluid between parallel plates of infinite width subject to a pressure gradient, $d P / d x$. Accordingly, an analytical solution exists where $\mu$ is the fluid viscosity.

$$
u(y)=\frac{1}{2 \mu}\left[y^{2}-\frac{h^{2}}{4}\right] \frac{d P}{d x}
$$

Although relation (3.2) incorrectly describes the concavity of the profile of the advancing front, the relationship between velocity versus a pressure gradient correctly represents the physics of the surface-directed microfluidic platform. Moreover, since the curvature of the front is indiscernible from a macroscopic perspective, one can assume the centerline velocity is an adequate approximation for the overall fluid filament velocity.

$$
u(0) \approx u=\frac{-h^{2}}{8 \mu} \frac{d P}{d x}
$$

Neglecting edge effects, it is possible to utilize the Laplace pressure as an approximate pressure drop dependent upon the fluid surface tension $\gamma$ and the advancing contact angle $\theta$ assuming contact angle dynamics are negligible.

$$
\frac{d P}{d x} \approx \frac{\Delta P}{x}=\frac{-2 \gamma \cos \theta}{h}
$$

Incorporating relation (3.4) into relation (3.3), an analytical expression for the velocity is established.

$$
u=\frac{h \gamma \cos \theta}{4 \mu x}
$$

Integrating relationship (3.5) with respect to $x$ from $O$ to $L$ with respect to time from $O$ to $t$ applying the initial condition of a distance of $x=0$ at $t=0$ results in an analytical relationship for position as a function of time.

$$
L(t)=\left[\frac{h \gamma \cos \theta}{2 \mu} t\right]^{1 / 2}
$$


This result agrees with the model posed by Yang (Yang et al., 2004), and demonstrates the $t^{1 / 2}$ dependency on position demonstrated for capillary-driven microfluidic platforms of alternative geometry (Mann Jr et al., 1995, Romero \& Yost, 1996, Rye, Mann et al., 1996, Rye, Yost et al., 1996, Zhmud et al., 2000) or fabrication approach (Bouaidat et al., 2005).

From result (3.6), one finds that a characteristic diffusion constant, $D$, exists for this system dependent upon system architecture, fluid properties and fluid-substrate energetic interactions.

$$
D=\frac{h \gamma \cos \theta}{2 \mu}
$$

For pure water at $20^{\circ} \mathrm{C}$ in contact with the PVC material, $D$ is $5.6 \times 10^{-4} \mathrm{~m}^{2} / \mathrm{s}$ and $1.1 \times 10^{-3}$ $\mathrm{m}^{2} / \mathrm{s}$ for gap heights of $116.4 \mu \mathrm{m}$ and $229.0 \mu \mathrm{m}$, respectively.

Results were attained utilizing 10 independent devices patterned with $18 \%(\mathrm{w} / \mathrm{w})$ ink solutions with the three largest path widths (i.e. large widths chosen to eliminate inconsistencies associated with misalignment of the visually assembled devices). The results indicate good agreement with the theoretical model (figure 3.9). Figures 3.9a and 3.9b demonstrate the relationship of distance as a function of time. The theoretical model was also implemented with one standard deviation in the contact angle of the PVC material. The experimental data was also linearized for figures $3.9 \mathrm{c}$ and $3.9 \mathrm{~d}$ in order to exhibit the deviation in time measurements as the fluid progressed a specified distance. 


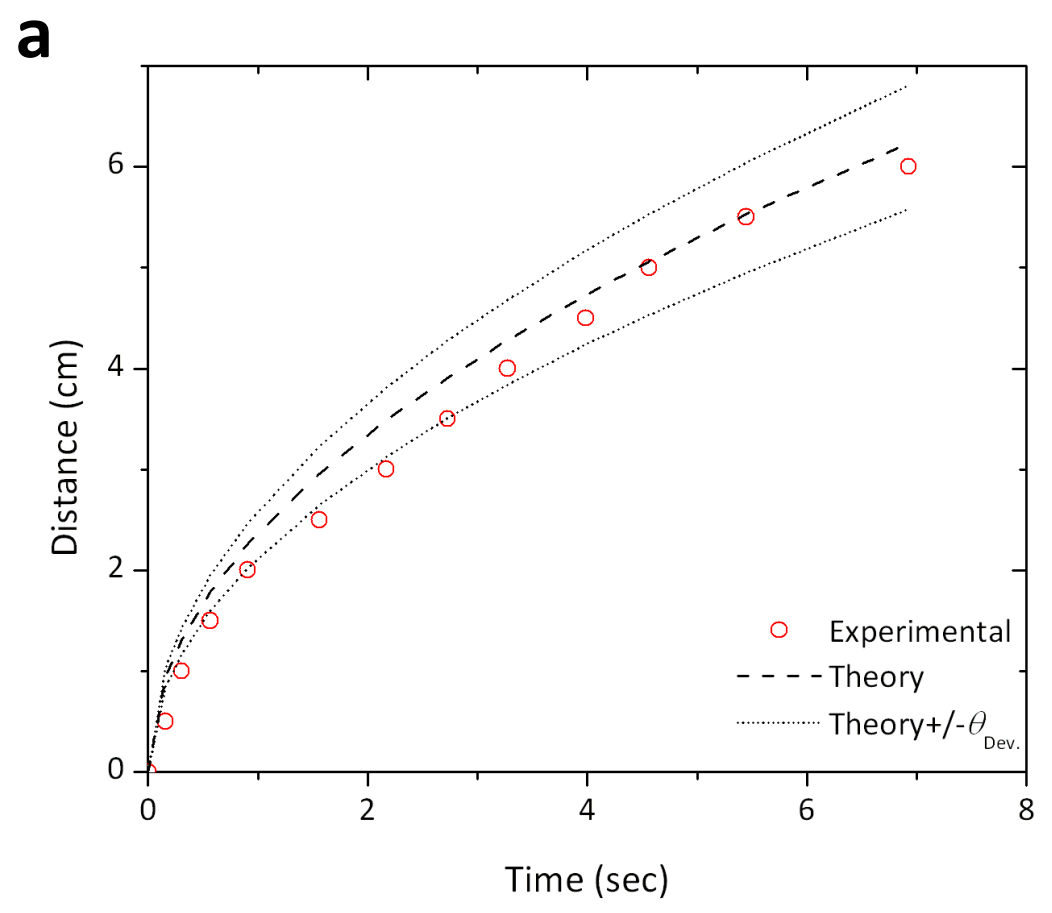

Figure 3.9a Capillary dynamics as a function of time and system architecture for a height of 116.4 micrometers and a contact angle of $82.4^{\circ} \pm 1.5^{\circ}$.

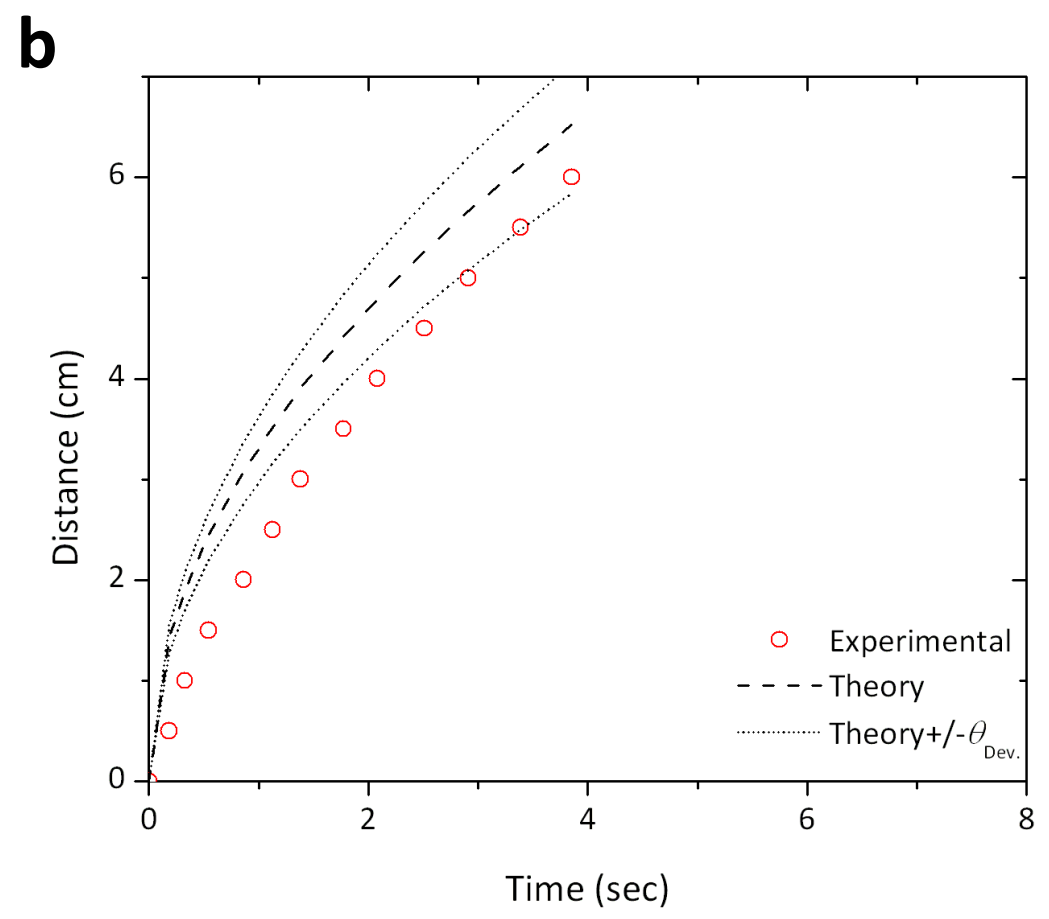

Figure 3.9b Capillary dynamics as a function of time and system architecture for a height of 229.0 micrometers and a contact angle of $82.4^{\circ} \pm 1.5^{\circ}$. 


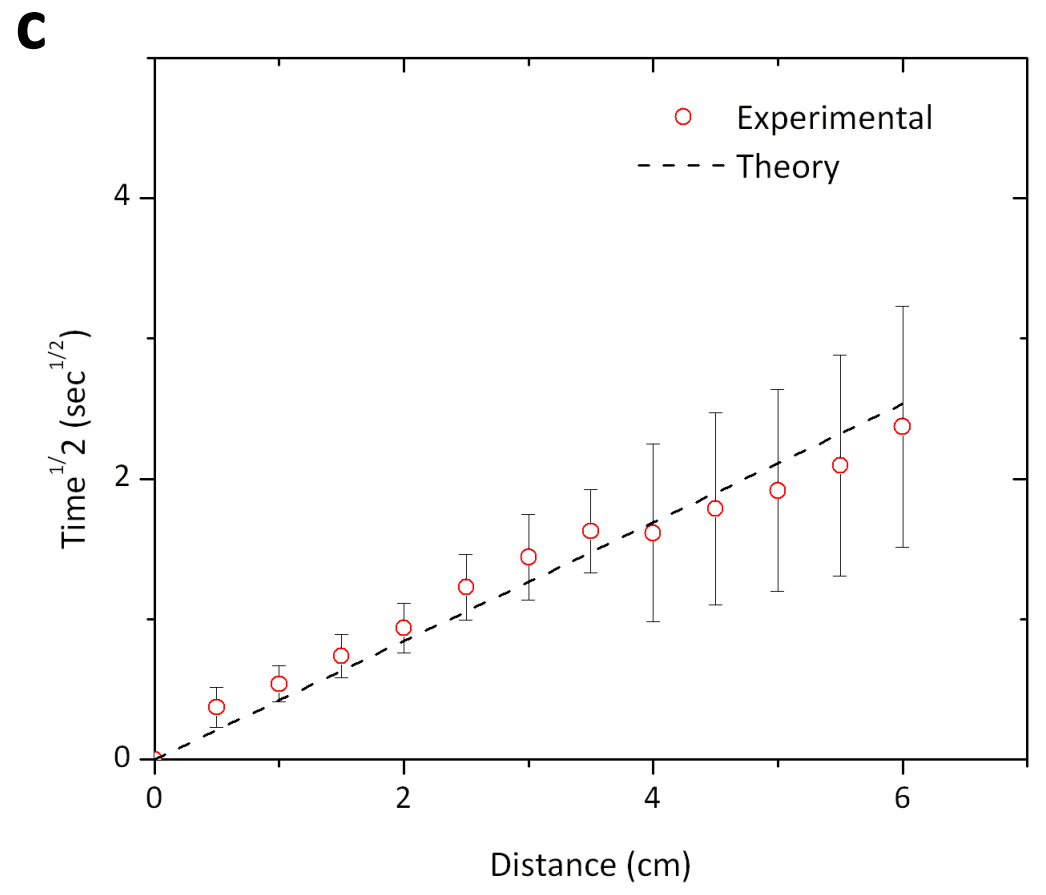

Figure 3.9c Linearized capillary dynamics as a function of time and system architecture for a height of 116.4 micrometers and a contact angle of $82.4^{\circ}$.

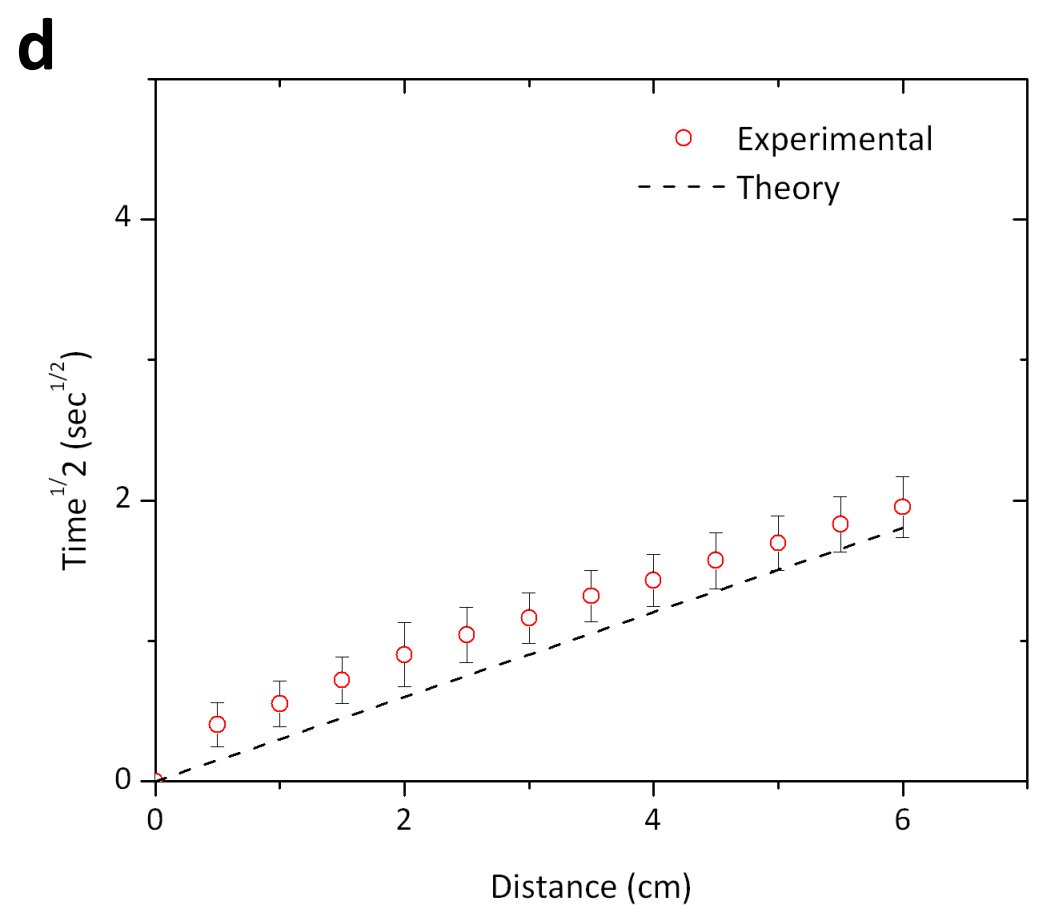

Figure 3.9d Linearized capillary dynamics as a function of time and system architecture for a height of 229.0 micrometers and a contact angle of $82.4^{\circ}$. 
As predicted by expression (3.6), the meniscus position is not directly dependent upon the path width. Moreover, the system dynamics are dependent upon the hydrophobic contact angle rather than the hydrophilic contact angle. This result seems fortuitous at first. However, this is an implication of choosing a hydrophobic material with a contact angle less than $90^{\circ}$. Specifically, the outside layer of the fluid is able to contact the hydrophobic region and become 'pinned'. For chemically heterogeneous surfaces, metastable equilibrium states can occur resulting in a multitude of contact angles to be realized where chemical heterogeneities arise (Neumann \& Good, 1972). In particular for the STCM device, the fluid front progressing across the hydrophilic region should have a contact angle of approximately $60.0^{\circ} \pm 0.3^{\circ}$. However, as the fluid front advances and comes into contact with the PVC material, pinning occurs on the hydrophilic-hydrophobic region resulting in the manifestation of a contact angle of approximately $82.4^{\circ} \pm 1.5^{\circ}$ limiting the ability to proceed across the hydrophilic conduit at a high velocity. A good review of the theory of contact angle pinning can be found in the literature (Leger \& JoannyS, 1992) although a comprehensive characterization of pinning in the STCM platform is not necessary in understanding the physical phenomenon occurring in a qualitative sense. Consequently, the progression of the meniscus is rate limited by the maximum velocity that can be achieved for a fluid progressing across the hydrophobic PVC substrate material.

Furthermore, considering the a characteristic length of a hydraulic diameter (i.e. four times the cross-sectional area divided by the perimeter) and differentiating relation (3.6) with respect to time allows for the development of a relation for the dynamic Reynolds number.

$$
\operatorname{Re}=\frac{\rho}{\mu} \frac{w h}{(w+h)} \frac{D}{\sqrt{D t}}
$$


The resulting proportionality of velocity to the inverse of the square-root of time is consistent with results previously published (Chen et al., 2006). Expression (3.8) can be solved to find the amount of time required for the Reynolds number to drop into the laminar regime ( $\operatorname{Re} \leq$ 2000). For the system capable of the most rapid flow, with a gap height of $229.0 \mu \mathrm{m}$ and width of $1352.1 \mu \mathrm{m}$, the time requirement to achieve laminar flow in the surface-directed device is 10.5 microseconds indicating fully developed flow is achieved quickly. This suggests that the assumption of negligible contact angle dynamics is plausible. Furthermore, this result indicates that capillary flow alone does not result in significant mixing within the device.

Furthermore, employing the Monge representation, it is possible to calculate the curvature associated pressure jump, $[p]$, across the advancing interface enabling the quantification of the capillary driving force at every point upon the advancing free surface. Specifically, using the general Laplace equation, the pressure jump is related to the fluid surface tension and mean curvature $H$, viz.

$$
[p]=2 \gamma H
$$

Consequently, calculating the curvature of the interface directly enables the determination of the pressure jump at any point upon the fluid-gas interface as opposed to utilizing theLaplace pressure approximation where the maximum and minimum curvature are represented as constants (Delamarche et al., 1998, Juncker et al., 2002). However, before this can be done, the Monge representation must be implemented mapping a 2D surface to a 3D surface (Mann $\mathrm{Jr}, 2002)$.

$$
\Sigma_{\mathrm{t}}:=\left\{\begin{array}{c}
x=u^{1} \\
y=u^{2} \\
z=\zeta\left(u^{1}, u^{2}, t\right)
\end{array}\right.
$$


The two dimensional surface is a rectangular patch of dimensions $-w / 2 \leq u^{1} \leq w / 2$ and $-h / 2 \leq u^{2} \leq h / 2$ which is mapped to a three dimensional curved surface of the advancing fluid meniscus. Assuming the surface is sufficiently smooth, as mandated by physical energetics, and that the surface can be approximated adequately by using the minimum and maximum radii of curvature (this is achieved by assuming that the curvature in the $x$ and $y$ directions are dis-joint and using a circle function of a defined radius to fit the curvature of each direction), it is possible to fit the advancing surface to two functions each contributing to the surface curvature independent of the other $(L(t)$ is the dynamic contribution directly from expression 3.6).

$$
\begin{gathered}
\zeta_{1}\left(u^{1}\right)=-\frac{w}{2}+\left[\frac{w^{2}}{4}-\left(u^{1}\right)^{2}\right]^{1 / 2} \\
\zeta_{2}\left(u^{2}\right)=\frac{h}{2 \cos \theta}+\left[\frac{h^{2}}{4(\cos \theta)^{2}}-\left(u^{2}\right)^{2}\right]^{1 / 2} \\
\zeta\left(u^{1}, u^{2}, t\right)=L(t)+\zeta_{1}\left(u^{1}\right)+\zeta_{2}\left(u^{2}\right)
\end{gathered}
$$

For $\zeta_{2}\left(u^{2}\right)$ the contact angle considered is the hydrophilic material contact angle across the width of the path, $w$. In this approach, the Monge model implicitly assumes the hydrophobic region has a contact angle of $90^{\circ}$. Therefore, utilizing this idealization, the pressure jump does not depend on contact angle competition.

Consequently, the advancing front can be reconstructed for any given time via mapping $\mathbb{R}^{2} \rightarrow \mathbb{R}^{3}$. Furthermore, the mean curvature of the surface can be explicitly calculated where the subscripts denote the spatial derivative taken for each associated term.

$$
H=\frac{\left(1+\zeta_{u^{1}}^{2}\right) \zeta_{u^{2} u^{2}}-2 \zeta_{u^{1}} \zeta_{u^{2}} \zeta_{u^{1} u^{2}}+\left(1+\zeta_{u^{2}}^{2}\right) \zeta_{u^{1} u^{1}}}{2\left(1+\zeta_{u^{1}}^{2}+\zeta_{u^{2}}^{2}\right)^{3 / 2}}
$$


Considering a fluid meniscus advancing upon the hydrophilic cellulose acetate material, the curvature based pressure jump has been explicitly calculated. Figure 3.10 demonstrates the structure of the advancing fluid front for the four architectures investigated in this study. Figure 3.11 and table 3.2 summarize the calculated results for interfacial pressure jump versus the approximated result.
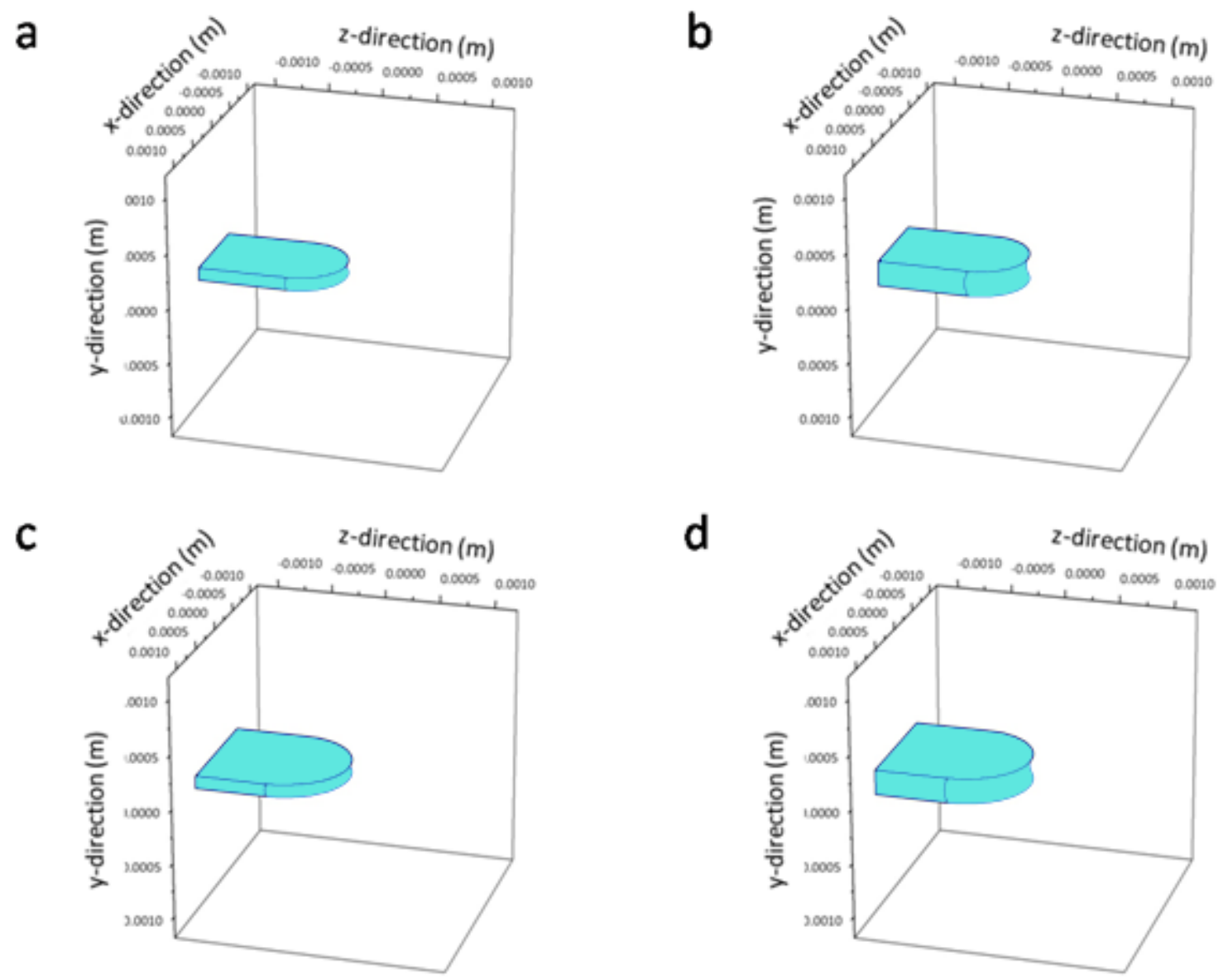

Figure 3.10 Reconstructed surfaces utilizing the Monge surface representation. a $w=830.9$ $\mu \mathrm{m}, h=116.4 \mu \mathrm{m} \mathrm{b} w=830.9 \mu \mathrm{m}, h=229.0 \mu \mathrm{m} \mathbf{c} w=1146.9 \mu \mathrm{m}, h=116.4 \mu \mathrm{m} \mathbf{d} w=$ $1146.9 \mu \mathrm{m}, h=229.0 \mu \mathrm{m}$. 
a

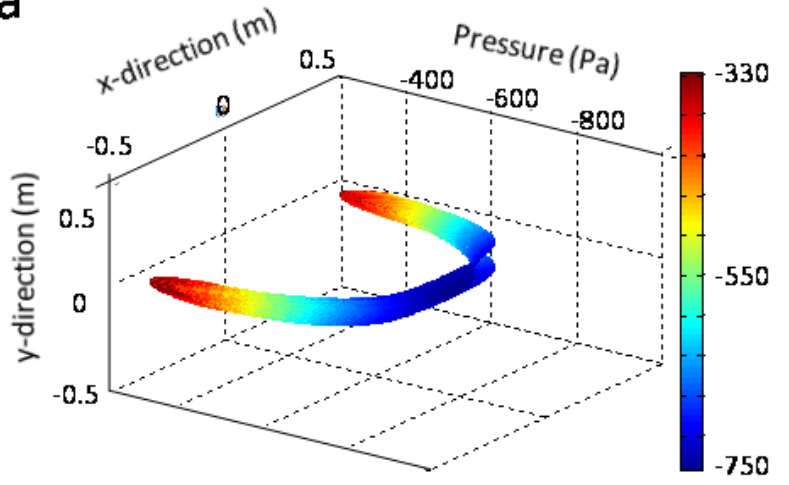

C

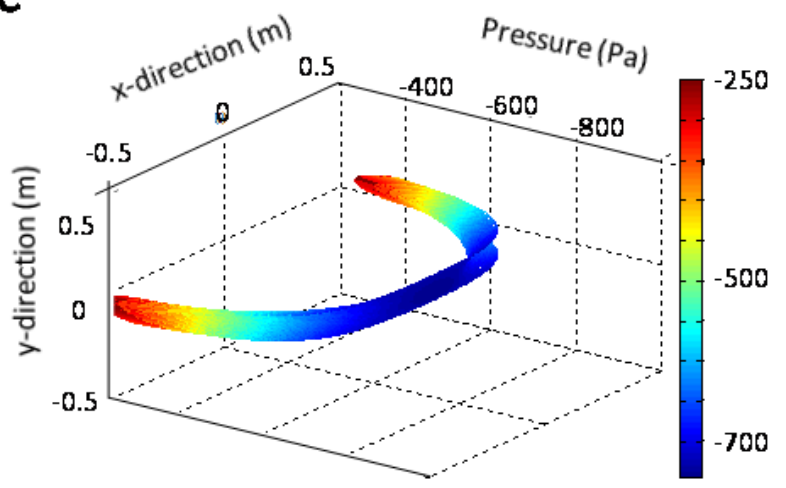

b

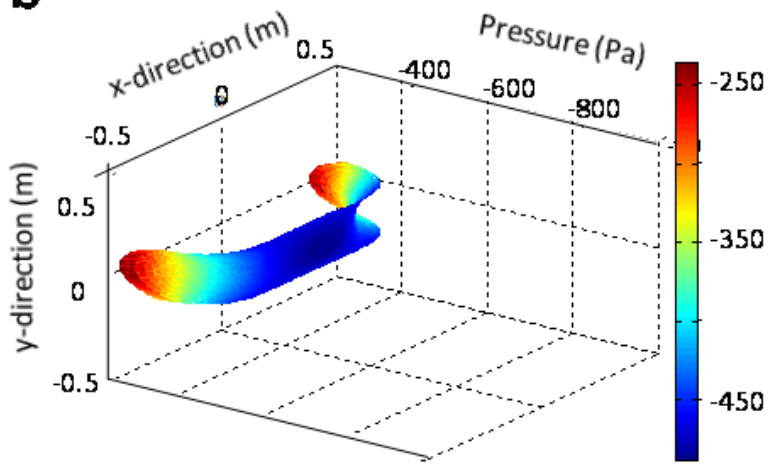

d

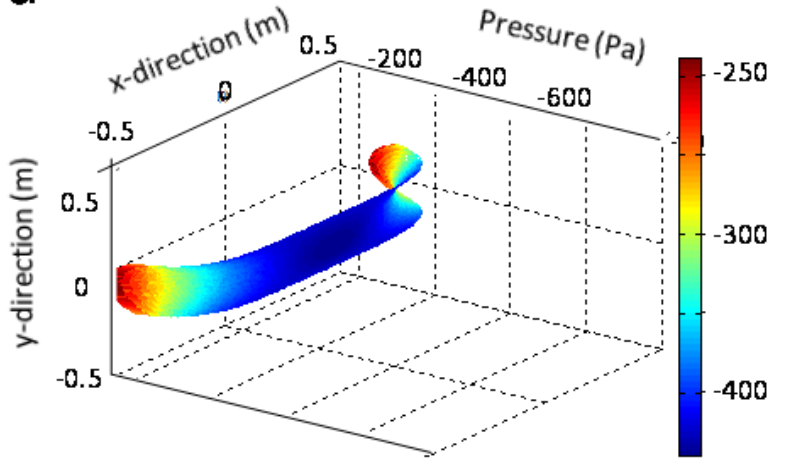

Figure 3.11 Predicted pressure jump across gas-liquid interface for all configurations investigated. a $w=830.9 \mu \mathrm{m}, h=116.4 \mu \mathrm{m} \mathbf{b} w=830.9 \mu \mathrm{m}, h=229.0 \mu \mathrm{m} \mathbf{c} w=1146.9 \mu \mathrm{m}$, $h=116.4 \mu \mathrm{m} \mathbf{d} w=1146.9 \mu \mathrm{m}, h=229.0 \mu \mathrm{m}$.

Table 3.2 Summary of calculations and comparison to the Laplace pressure approximation.

\begin{tabular}{|c|c|c|c|c|c|}
\hline $\begin{array}{c}\text { Gap Height } \\
\text { (micrometers) }\end{array}$ & $\begin{array}{c}\text { Path Width } \\
\text { (micrometers) }\end{array}$ & $\begin{array}{c}\text { Laplace } \\
\text { Approximation } \\
\text { Pressure Jump } \\
(\text { Pa) }\end{array}$ & $\begin{array}{c}\text { Averaged } \\
\text { Curvature } \\
\text { Pressure Jump } \\
(\text { Pa) }\end{array}$ & $\begin{array}{c}\text { Minimum } \\
\text { Pressure Jump } \\
(\text { Pa) }\end{array}$ & $\begin{array}{c}\text { Maximum } \\
\text { Pressure Jump }\end{array}$ \\
\hline 116.4 & 830.9 & -641.5 & -683.8 & -791.8 & -297.0 \\
\hline 116.4 & 1146.4 & -337.3 & -430.5 & -487.7 & -236.2 \\
\hline 229.0 & 830.9 & -635.2 & -637.6 & -744.0 & -249.3 \\
\hline 229.0 & 1146.4 & -331.0 & -383.9 & -439.9 & -188.4 \\
\hline
\end{tabular}

The approximated pressure jump is computed directly from the Laplace pressure approximation where is $\theta_{P}$ is the contact angle of the polar hydrophilic material and $\theta_{N}$ is the 
contact angle of the non-polar hydrophobic material (Delamarche et al., 1998, Juncker et al., 2002).

$$
\Delta P=2 \gamma\left[\frac{\cos \theta_{P}}{h}+\frac{\cos \theta_{N}}{w}\right]
$$

The averaged pressure jump is a length-normalized Frobenius norm of the discretized $n \times n$ pressure jump matrix.

$$
[p]_{\text {Avg. }}=\frac{1}{n}\left[\sum_{j=1}^{n} \sum_{i=1}^{n}[p]_{i, j}^{2}\right]^{1 / 2}
$$

As demonstrated by table 3.2, the curvature-based calculations are in good agreement. Moreover, as the height decreases, the curvature-based calculation begins to converge upon the Laplace result. This result arises from the fact that as height decreases, the hydrophilic contact angle term begins to dominate in the pressure jump calculation.

The higher-dimensional mapping technique produces a smooth surface which can be exploited for the quantitative characterization of the driving force for the microfluidic system. The method yields a position-dependent pressure jump as opposed to the constant Laplace pressure approximation. Consequently, the Monge surface mapping technique is a more accurate depiction of the true physics involved with surface-directed microfluidic capillary transport.

\subsubsection{Thick Film Printing of Three-Dimensional Microfluidic Systems}

As the thick film printing process results in films of finite thickness (see figure 3.8), this patterning method can be exploited to produce three-dimensional microfluidic devices where the printed film acts as the sides of the fluidic channel. The advantage of this approach 
is that it can result in stand-alone monolithic microfluidic devices fabricated with minimal rigor when compared to traditional three-dimensional microfluidics processing methods. For example, the production of three-dimensional structures in polydimethylsiloxane (PDMS) first requires the production of a negative stamp in a clean room environment involving the steps of substrate preparation, photoresist coating followed by baking, photomask alignment and light exposure, photoresist etching and development, and finally photoresist strip (Xia \& Whitesides, 1998). Furthermore, even after the master stamp is produced, the process of making microfluidic devices from the stamp is inherently a batch process as time is required for the liquid elastomer to cure before a device can be removed from the stamp.

Alternatively, three dimensional structures can be with much less rigor via thick film printing. Again, a one time screen making process is required as described in section 3.2.2; however, this process is significantly less cumbersome than the preparation of a master stamp. Moreover, once a screen has been produced, it can be used indefinitely if proper maintenance is conducted pre/post-patterning. Lastly, as curing time is not required before subsequent devices are made, this process can be performed continuously

Patterning of three-dimensional devices is slightly different than in the case of the two-dimensional analogs. First, a hydrophilic (or in the worst case, moderately hydrophobic) substrate material is selected to be patterned (e.g. poly(methyl methacrylate), polycarbonate, glycol-modified poly(ethylene terephthalate), et al.). Once the substrate material has been selected, it is most convenient to choose an polymer for the ink system that can be dissolved in a common solvent as the substrate material to function as the walls and spacers for the microfluidic system as well as facilitating solvent welding of the ink and substrate to ensure the system is hermetically sealed. In addition to choosing an ink material with a common 
solvent, it is also important to choose an ink material with a drastically different surface energy/tension than the substrate to be patterned. The consequence of not following this criteria is that the patterned ink will wet the substrate resulting in the loss of the pattern as the substrate cures. For this reason, it is not possible to pattern a substrate with an ink made from the same substrate material (e.g. it is not possible to pattern a polycarbonate substrate with polycarbonate dissolved in tetraethylene glycol dimethyl ether or wetting will occur and resolution will be lost).

Following the described manufacturing methodology, several devices are fabricated supporting autonomous fluid transport in a three-dimensional microfluidic network. Material systems successfully demonstrated include patterning 30\% (w/w) polystyrene dissolved in tetraethylene glycol dimethyl ether upon both poly(methyl methacrylate) (PMMA) and polycarbonate. Following patterning, the device is allowed to cure overnight with weight placed on top to ensure solvent wetting occurs $(\sim 0.5 \mathrm{~kg})$.

In addition to demonstrating this manufacturing method, an assay has been integrated within this platform for the colorimetric detection of glucose. The polystyrene ink is patterned through a screen upon a PMMA substrate. An additional PMMA substrate is placed upon the patterned substrate while the ink is still wet enabling solvent wetting of the polystyrene ink to the PMMA substrates. The particular pattern (figure 3.12) enables the introduction of reactants (potassium iodide and 1:5 ratio of horseradish peroxidase:glucose oxidase - assay discussed in depth in section 3.3.2) into a tortuous chamber. The reactant solution is allowed to evaporate overnight. Following the introduction of reactant, the fluid sample with $0.5 \mathrm{M}$ glucose is introduced to the inlet the glucose solution is transported via capillarity to the tortuous reaction zone where interaction with the reagent mixture occurs. 
First, glucose is oxidized by the glucose oxidase in the presence of water and oxygen to give gluconic acid and hydrogen peroxide. The hydrogen peroxide is subsequently reduced to water by horseradish peroxidase with concomitant oxidation of iodide to iodine (Martinez et al., 2007). The reaction begins in the reactant inlet region and continues through the microfluidic plug flow reactor region where the dried reagents solublize and react with the liquid sample serum. The reacted liquid then proceeds to the detection zone where the yellow iodine is visible in comparison to the transparent control detection zone (figure 3.13). An invention disclosure has been filed with regard to this approach to fabricating threedimensional microfluidic reactors.

\section{a}

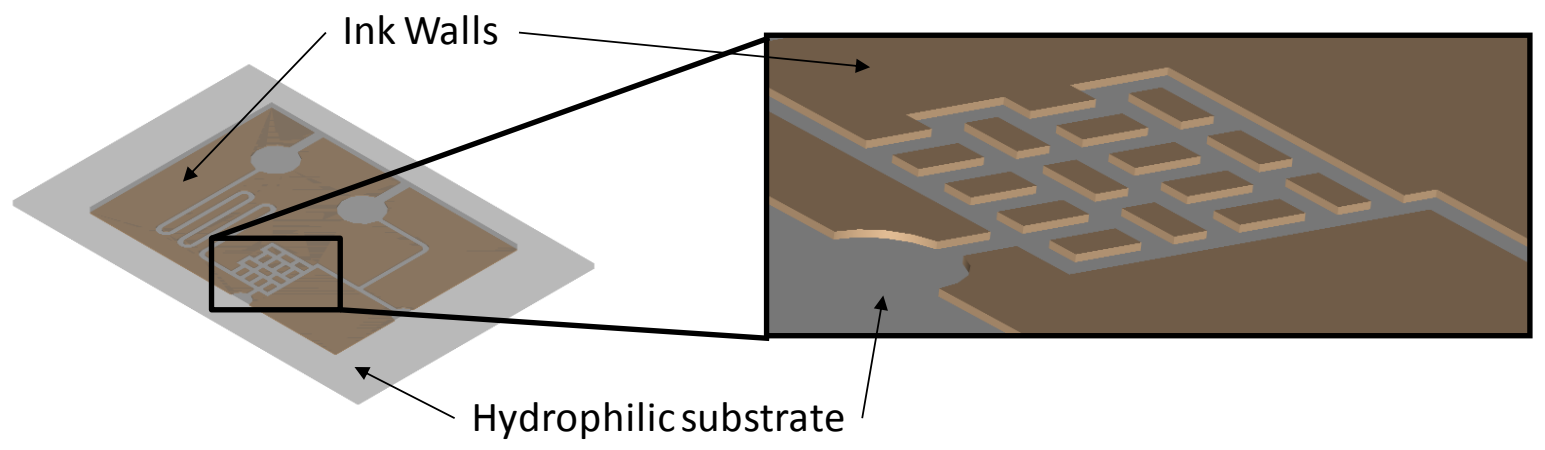

b

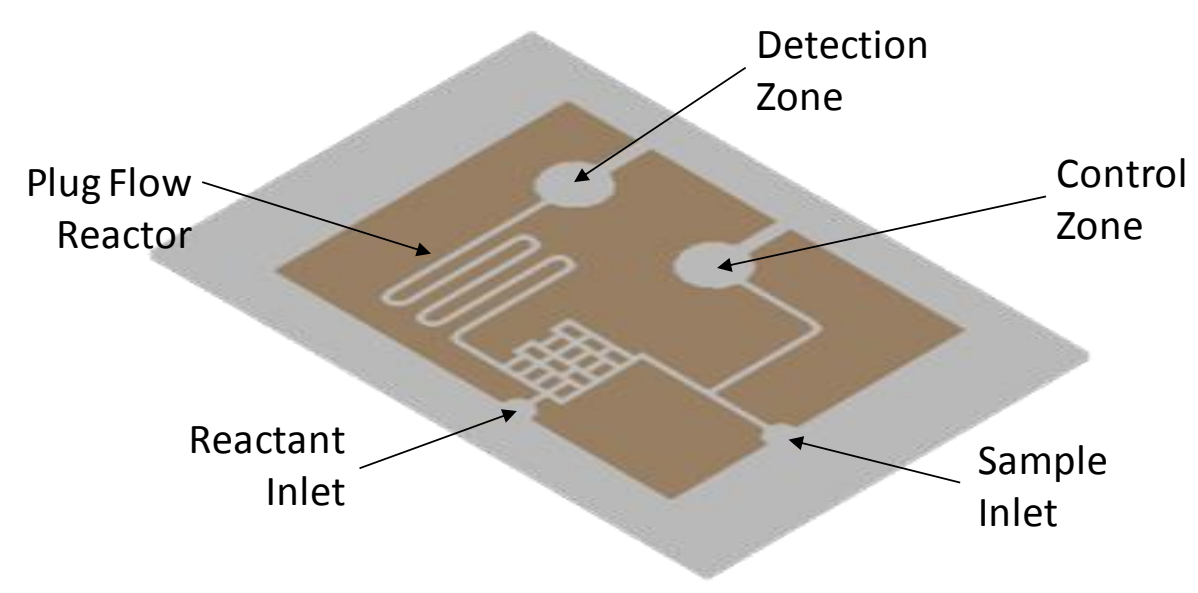

Figure 3.12 Three-dimensional microfluidic device design capable of facilitating the colorimetric detection of glucose. a Distinguishing ink from substrate and an enhanced view 
of the tortuous reaction chamber responsible of bringing glucose and reagents into intimate contact. b Annotation of function of designed pattern.

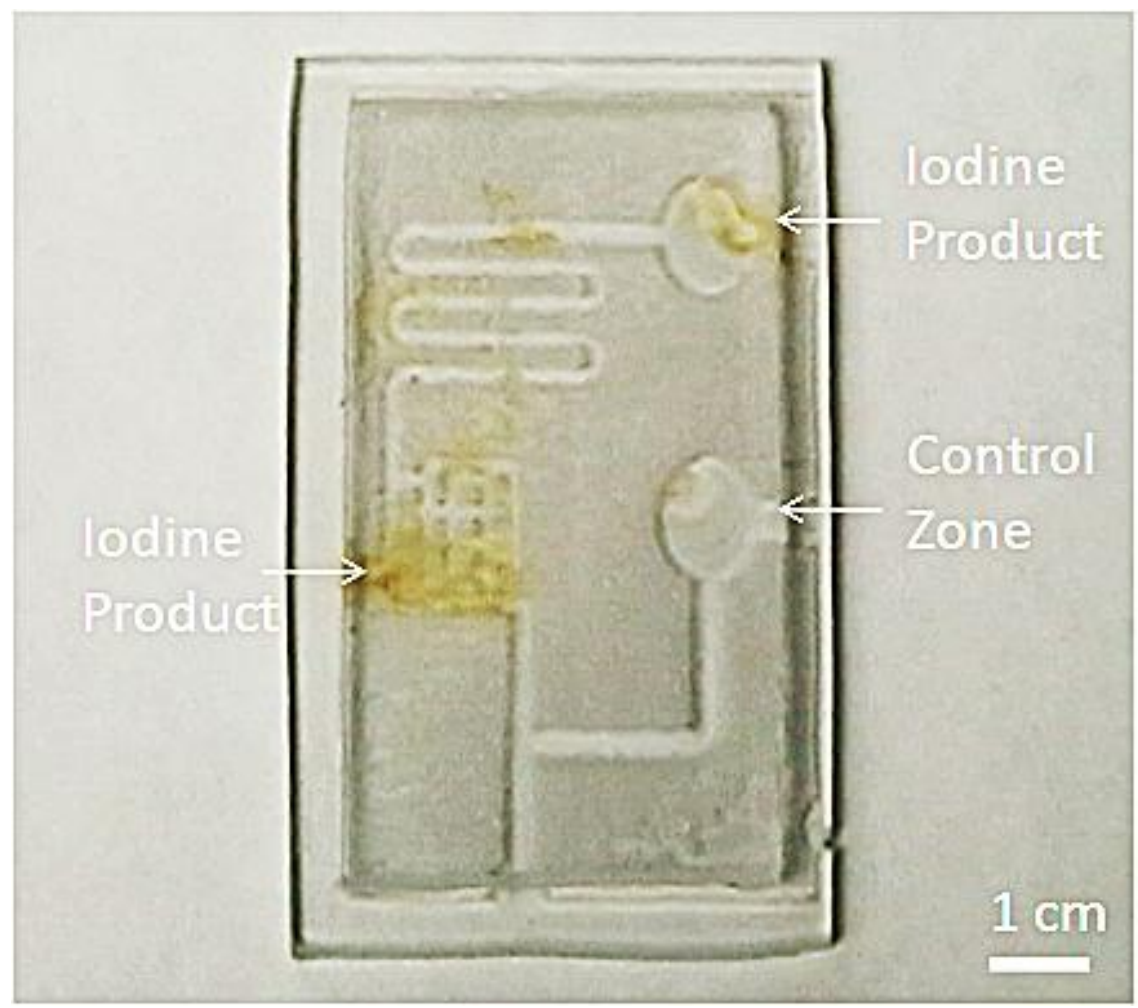

Figure 3.13 Demonstration of glucose assay within three-dimensional microfluidic platform.

\subsection{Application of Platforms}

In conjunction with the development of microfluidic fabrication processes involving minimal rigor and expense, platform applications are investigated. It is of the opinion of the investigators of this work that novel fabrication approaches alone are not enough to garner significant attention to STCM platforms for potential commercialization. As a result, several applications are under investigation demonstrating the efficacy of STCM platforms to conduct lab-on-a-chip processes. Specifically, this section details the incorporation of 
enzymatic catalysis, on-chip urinalysis and microfluidic mixing within the surface tensionconfined microfluidic platforms.

\subsubsection{Enzymatic Catalysis}

Having established patternability for the sol gel system, efforts were made to demonstrate the incorporation of a biological assay and the develop methodology for the extension toward more complicated biological analyses. An assay is chosen based upon the availability and cost of reagents as well as the ease at which the assay can be executed. This demonstration is fulfilled by the hydrolysis of O-nitrophenyl- $\beta$-D-galactopyranoside to Onitrophenol and D-galactose in the presence of $\beta$-D-galactosidase doped sol gel glass. Matrix doping is achieved via an alternative sol gel preparation pathway utilizing tetralkyl orthosilicate precursors based on literature results (Ellerby et al., 1992).

In order to synthesize the enzyme-doped sol gel glass, one first prepares a solution of $86.5 \%(\mathrm{v} / \mathrm{v})$ tetraethyl orthosilicate (Sigma Aldrich) in water. Next, add $1 \%$ of the total solution volume of $0.1 \mathrm{M} \mathrm{HCl}$ as catalyst. The reaction mixture is sonicated for 20 minutes to form a dispersion of the orthosilicate gel precursor in the water. It is next necessary to add $100 \%$ of total solution volume of phosphate buffered saline solution of desired $\mathrm{pH}$ (e.g. $\mathrm{pH}=$ 7.4 for the enzyme of interest in this study). An additional sonication step is carried out for 5 minutes to ensure the added buffer solution is included in the dispersed state. The enzyme component is added to the sonicated solution to at the desired concentration $(0.42 \mathrm{mM}$ of $\beta$ D-galactosidase) and agitated accordingly to distribute the enzyme throughout the solution. The solution is then applied to the substrate promptly before the sonicated dispersion begins to separate into immiscible phases. The substrate is incubated at $37^{\circ} \mathrm{C}$ (or the temperature of 
optimal activity for the particular enzyme of interest) for 1 hour at $100 \%$ relative humidity. After incubation, the biological component should be entrapped in a nanoporous gel in the most biologically active conformation. This assay is demonstrated to elicit a response to the presence of $14 \mathrm{mM}$ O-nitrophenyl- $\beta$-D-galactopyranoside in sol gel doped glasses as well as incorporated into the sol gel assay (figure 3.14).

(A)

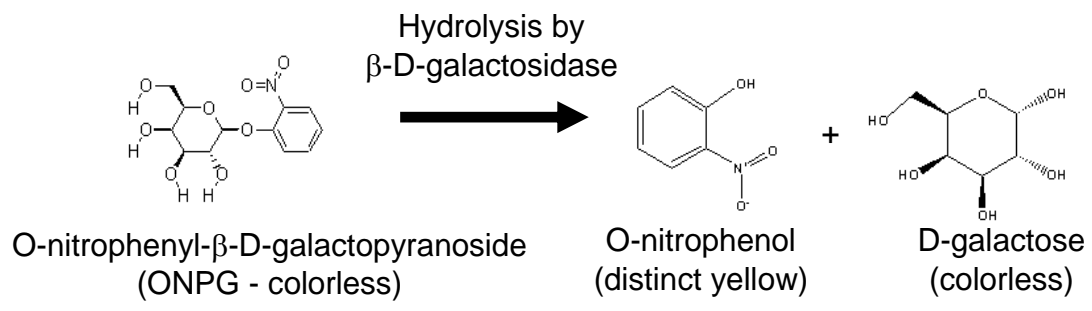

(B)

Hydrolysis by

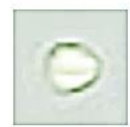
$\beta$-D-galactosidase
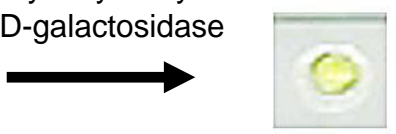

(C)

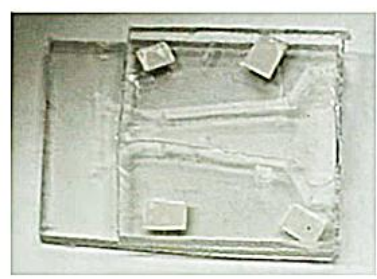

Hydrolysis by $\beta$-D-galactosidase

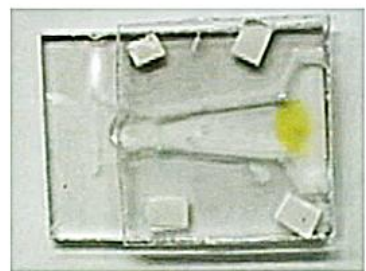

Figure 3.14 (A) Reaction of substrate with substrate in presence of enzyme to produce a distinct yellow product. (B) Demonstration of the assay with a droplet of substrate solution upon a spotted 'dot' of encapsulated enzyme. (C) Reaction incorporated with STCM device ( $\sim 50 \mu \mathrm{l}$ of substrate solution)

The kinetics of the reaction are rather fast and the yellow response is apparent within a matter of seconds. The evolution of color begins to slow and eventually stops altogether within approximately 15 minutes in the microfluidic platform. The chip capable of enzymatic catalysis not only demonstrates simple on-chip reaction processes are capable within the STCM but also exhibit protocols exist for incorporating $\mathrm{pH}$ and temperature sensitive 
biomolecules within the platforms. This approach can easily be extended to a wide variety of enzymes and other biomolecules.

\subsubsection{On-chip Urinalysis}

Monitoring the health is essential to ensure the well-being of an individual has not been compromised. However, seeking trained personnel in a clinical setting is not always feasible or financially responsible. This is particularly true for rural populations and third world countries where clinics are few and far between. Consequently, there is a great opportunity for commercialization for inexpensive and automated analyses capable of monitoring the physical condition of an individual.

One aspect of health monitoring is to consider the glucose and albumin levels within an individual's biological fluids. This can be achieved easily through urinalysis. An example of automating this analysis has been demonstrated on photolithographically-patterned chromatography paper (Martinez et al., 2007). The bioassays can produce a qualitative colorimetric response for the detection of biologically relevant concentrations of both glucose $(2.5-500 \mathrm{mM})$ and albumin $(0.38-75 \mu \mathrm{M})$. Although the devices are capable of automating the urinalysis process, bioassay manufacture is involved and time intensive including steps such as (i.) chromatography paper photoresist soak (ii.) photomask alignment and pre-bake (iii.) UV exposure and post-bake (iv.) resist development and propanol wash (v.) plasma oxidation (vi.) device cut-out and (vii.) spotting of reagents. Again, since several baking steps and the load-unload plasma oxidation step are involved, the photolithographic procedure is inherently a batch process. Additionally, since chromatography paper is opaque, the opportunity to couple the analysis with analytical equipment (e.g. photospectrometery) is 
eliminated. Consequently, an opportunity for improvement exists in both mitigating time involved with device fabrication as well as finding a transparent platform capable of supporting optical equipment for quantitative results.

The disadvantages of assay fabrication photolithographically on chromatography paper presents the opportunity for improvement of this analysis within the STCM platform. Not only are the manufacturing methods much less involved but the optical properties of cellulose acetate paths patterned on PVC could enable quantitative results to be attained in the future. Such devices are fabricated according to the procedures described in section 3.2.2.

\section{Urine Analog}

An analogous solution to human urine was prepared following known protocols (Brooks \& Keevil, 1997, Martinez et al., 2007). The solutions include several acids and salts dissolved in distilled water. The suggested protocol for the preparation of $10 \mathrm{ml}$ of analog solution are summarized by table 3.3. The acid/salt solutions were supplemented with known quantities of glucose and bovine serum albumin (BSA) at biologically relevant concentrations ranging from $125-500 \mathrm{mM}$ with respect to glucose and 18.3-75 $\mu \mathrm{M}$ for albumin (i.e. bovine serum albumin for this study although the assay is non-specific to the organism from which it came). The $\mathrm{pH}$ of the solutions were adjusted to 6.0 using $1 \mathrm{M} \mathrm{HCl}$ and $1 \mathrm{M} \mathrm{NaOH}$ and an Oakton $\mathrm{pH} 1100$ bench-top meter. The prepared solutions are stored at $4{ }^{\circ} \mathrm{C}$ when not in use. 
Table 3.3 Composition of urine analog prepared for on-chip urinalysis (mass calculated for $10 \mathrm{ml}$ of total solution).

\begin{tabular}{|c|c|c|c|c|}
\hline & Compound & Concentration (mM) & Mol. Wt. (g/mol) & Mass (g) \\
\hline \multirow{11}{*}{ 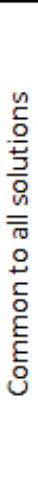 } & Lactic Acid & 1.10 & 90.08 & 0.0010 \\
\hline & Citric Acid & 2.00 & 192.12 & 0.0038 \\
\hline & Sodium Biocarbonate & 25.00 & 84.01 & 0.0210 \\
\hline & Urea & 170.00 & 60.06 & 0.1021 \\
\hline & Calcium Chloride & 2.50 & 110.98 & 0.0028 \\
\hline & Sodium Chloride & 90.00 & 58.44 & 0.0526 \\
\hline & Magnesium Sulfate & 2.00 & 120.36 & 0.0024 \\
\hline & Sodium Sulfate & 10.00 & 142.04 & 0.0142 \\
\hline & Potassium Phosphate Monobasic & 7.00 & 136.09 & 0.0095 \\
\hline & Potassium Phosphate Dibasic & 7.00 & 174.18 & 0.0122 \\
\hline & Ammomium Chloride & 25.00 & 53.49 & 0.0134 \\
\hline
\end{tabular}

Glucose Assay

The microfluidic device is spotted with reagent and the liquid is allowed to evaporate prior to utilization leaving the solid reagent adsorbed to the platform surface. The reagent solution includes $0.5 \mu \mathrm{l}$ of $0.6 \mathrm{M}$ potassium iodide (Sigma Aldrich) and $0.5 \mu \mathrm{l}$ of 1:5 ratio of horseradish peroxidase/glucose oxidase at a concentration of $12.8 \mathrm{units} / \mathrm{ml}$. The horseradish peroxidase/glucose oxidase reagent is prepared from a commercially available capsule and the associated protocol (Sigma Aldrich, Product Number G3660).

The glucose assay proceeds through the introduction of glucose to glucose oxidase in the presence of oxygen and water. The glucose is oxidized to gluconic acid and hydrogen peroxide. The hydrogen peroxide is then reduced to water by horseradish peroxidase with the simultaneous oxidation of iodide to iodine leaving a distinct brown product.

\section{Protein Assay}

Similarly to the glucose assay, the microfluidic device is spotted with the protein assay reagents and allowed to evaporate over time leaving solid reagent. The reagent solution includes $0.5 \mu \mathrm{l}$ of the indicator tetrabromophenol blue (Sigma Aldrich) dissolved in 95\% 
ethanol at a concentration of $3.3 \mathrm{mM}$. Furthermore, $0.5 \mu \mathrm{l}$ of $250 \mathrm{mM}$ citrate buffer $(\mathrm{pH}=$ 1.8) is spotted onto the microfluidic device. The citrate buffer is prepared by adding 47.3 $\mathrm{g} /$ liter of anhydrous citric acid (alternatively, $51.8 \mathrm{~g} / \mathrm{liter}$ is required if citric acid monohydrate is substituted for the anhydrous version) and $1.1 \mathrm{~g} /$ liter of sodium citrate. Although the initial $\mathrm{pH}$ of the citrate buffer solution is out of the range of $\mathrm{pK}$ values for citrate buffer $(\mathrm{pK}=[3.15,4.50,5.75])$, the buffer solution is prepared by according to previous implementation of this assay within a microfluidic device (Martinez et al., 2007).

The assay proceeds via a dye color change of tetrabromophenol blue from yellow to blue as the protein binds to the indicator (Pugia et al., 1999). Bovine serum albumin is used for the current study although the assay works identically for human serum albumin,

\section{Demonstration of Assay 'On-Chip'}

The assay is implemented in surface tension-confined microfluidic platforms via chip production as described in section 3.2.2. Path width is designed for a nominal value of 800 $\mu \mathrm{m}$ with a gap height of $229.0 \mu \mathrm{m}$. The devices required approximately $30 \mu \mathrm{l}$ of urine analog solution for implementation. Furthermore, reagents are spotted onto the chips as described and the urine analog produces color evolution at varying concentrations of glucose and BSA. Reaction time required less than an hour to proceed. Figure 3.15 demonstrates the results for a variety of concentrations. Although the concentrations of glucose and albumin utilized are slightly above physiological conditions, the strong colorimetric results in this proof of concept demonstration indicate that this platform has the capacity to facilitate on-chip detection processes. A physiological concentration analysis is currently in progress. 


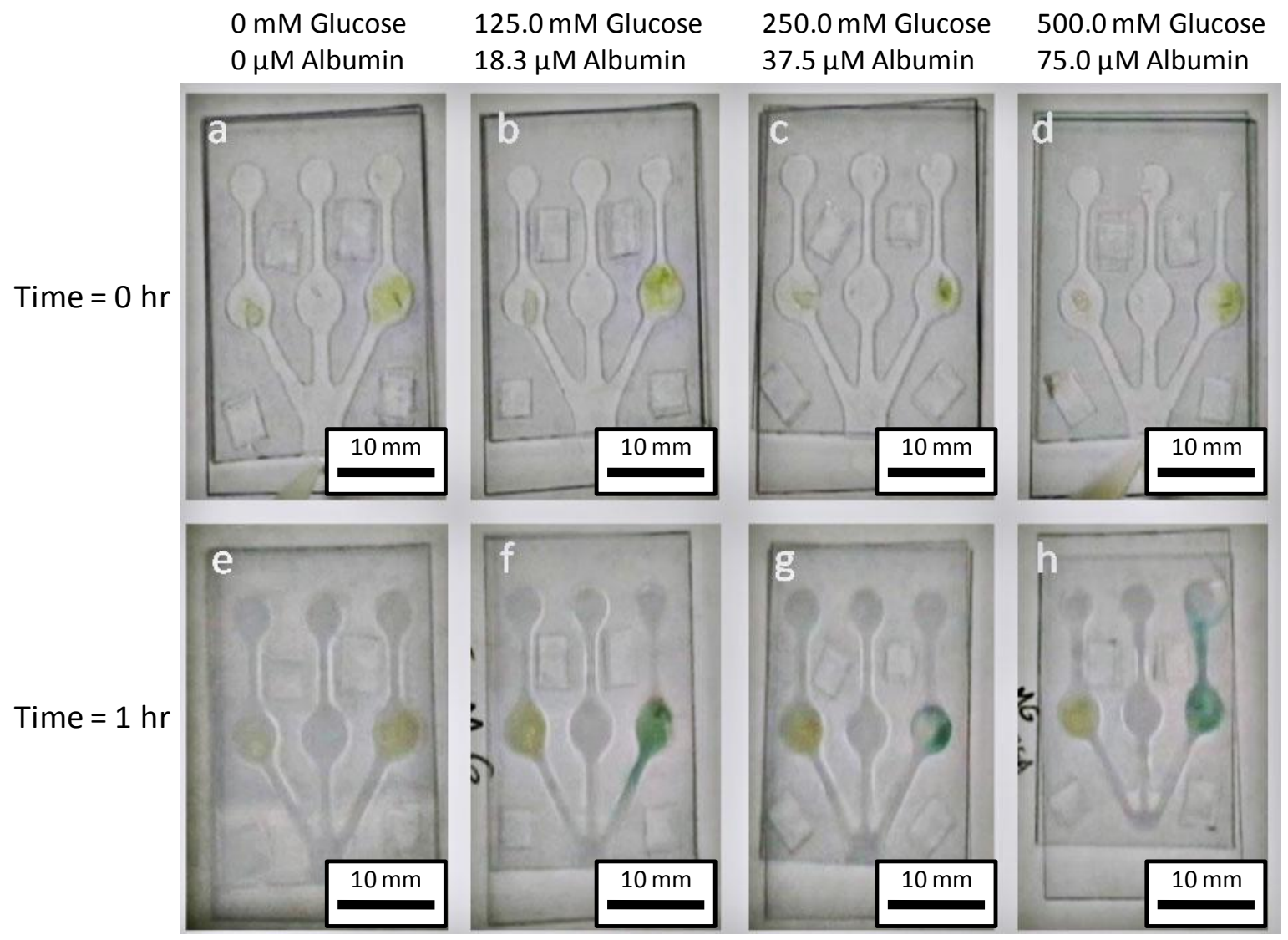

Figure 3.15 Initial assay results for a $0 \mathrm{mM}$ glucose and $0 \mu \mathrm{M}$ albumin, b $125 \mathrm{mM}$ glucose and $18.3 \mu \mathrm{M}$ albumin, $\mathbf{c} 250 \mathrm{mM}$ glucose and $37.5 \mu \mathrm{M}$ albumin, $\mathbf{d} 500 \mathrm{mM}$ glucose and 75 $\mu \mathrm{M}$ albumin. Final assay results for e $0 \mathrm{mM}$ glucose and $0 \mu \mathrm{M}$ albumin, f $125 \mathrm{mM}$ glucose and $18.3 \mu \mathrm{M}$ albumin, $\mathbf{g} 250 \mathrm{mM}$ glucose and $37.5 \mu \mathrm{M}$ albumin, $\mathbf{h} 500 \mathrm{mM}$ glucose and 75 $\mu \mathrm{M}$ albumin.

The strong presence of the blue color on the right hand side in the microfluidic platforms indicates the presence of albumin in chips b-d in contrast to the residual unreacted orange color in the control device (a). The yellow/brown color in the right hand side of the chips tend to be stronger for cases f-h than for $\mathbf{e}$. However, the color in platform $\mathbf{e}$ should not be mistaken as the production of new iodine. The color is a result of the dissolution of reagent spot seen on the left hand branch in 3.15a.

Results of the integration of an inexpensive and simple assay into the STCM platform demonstrate the facility the STCM chip has for manipulating fluids to encourage micro- 
reaction processes. Consequently, this result is encouraging for simple applications in which traditional clinical analyses are either too expensive for a patient or at a great inconvenience if clinics are at a limited number in a region or require significant travel to arrive there. Currently, investigation is in progress coupling glucose-albumin sensors with optical equipment for a quantitative response. In addition to this simple assay more powerful and versatile diagnostic techniques are currently under investigation for integration into the surface tension-confined microfluidic platform.

\subsubsection{Microfluidic Mixing}

Mixing is essential to bringing reactants within close enough proximity that a reaction process can occur. This is not a profound observation. However, in order to exploit microfluidic technology for 'lab on a chip' applications, adequate mixing mechanisms must be established in order to facilitate on-chip reaction processes.

Facilitating on-chip mixing is a complicated endeavor. Specifically, since the characteristic length scale (e.g. hydraulic radius, channel width or height, capillary tube radius) experienced within a microfluidic regime is on the order of micrometers, or even nanometers, the Reynolds number within such platforms is typically minimal indicating that microfluidic flow is inherently laminar (this conclusion can also be drawn from the low Péclet number).

Several strategies have been employed in the past to overcome problems associated with poor mixing within microfluidic devices. Such strategies may be active (e.g. driven via pressure disturbances, electrokinetic actuation, acoustic effects, et al.) or passive (e.g. driven through chaotic advection) (Nguyen $\& \mathrm{Wu}, 2005)$. The active approaches to driving micro- 
mixing generally require supporting equipment such as pumps, electrical supply sources, or resonators, further complicating chip design and leaving the chip susceptible to component malfunction and device failure.

Passive techniques generally implement obstacles in a microfluidic channel to drive micromixing through chaotic advection and striation of parallel streams. Johnson et al. achieved this through laser ablation of polycarbonate and glycol-modified polyethylene terephthalate for the formation of three-dimensional structures (Johnson et al., 2002). Stroock et al.used this approach to make structures in polydimethylsiloxane elastomers (Stroock et $a l ., 2002)$ and later extended this work to create virtual obstacles using active actuation with electrokinetic charge disturbances (Stroock \& Whitesides, 2003).

Furthermore, this approach has been modeled numerically and the advection process is understood. Wang et al. characterized mixing efficiency qualitatively using Poincaré maps and developed quantitative methods to determine channel length required for recirculation to occur (Wang et al., 2003). Camesasca et al. developed a method of quantifying mixing efficiency using Shannon entropy (Camesasca et al., 2006b, Camesasca et al., 2005). Camesasca et al. later conclude that utilizing periodically repeating obstacles does not truly result in chaotic mixing and instead suggest several geometries, developed with the Weierstrass fractal function, that can improve on mixing efficiency, for the staggered herring bone pattern in particular (Camesasca et al., 2006a).

The implementation of passive mixing through chaotic advection can be easily implemented within the STCM platform as well. As the flow within the STCM device is curvature-driven, the mediation of surface curvature yields an immediate means to manipulate velocity fields across a channel. This is achieved by patterning areas of increased 
curvature beside areas of minimal curvature thereby resulting in the acceleration and deceleration of the fluid within the STCM device. The patterning procedure is identical to that discussed in section3.2.2. Figure 3.16 demonstrates the concept of accelerating/decelerating the fluid surface through patterning of alternating areas of high and low surface energy.

(a)

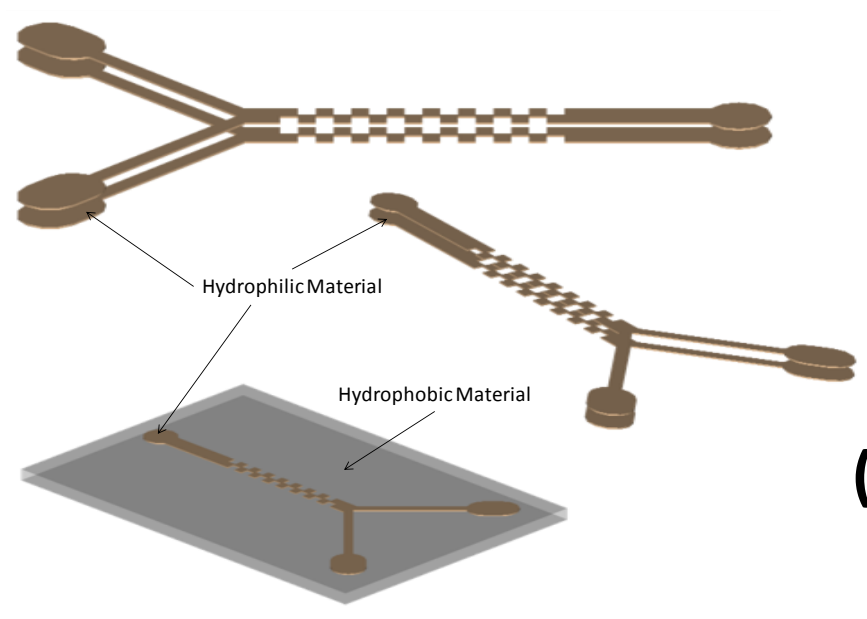

(b)

Control-no pattern

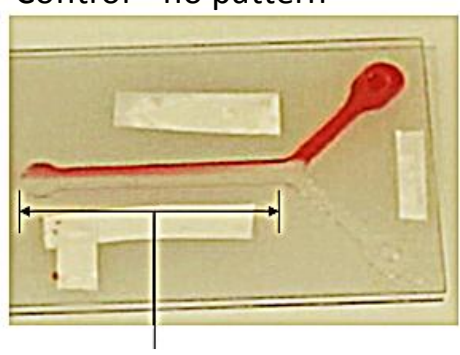

No Chaotic Advection Diffusive Transport

(c) Checker Pattern

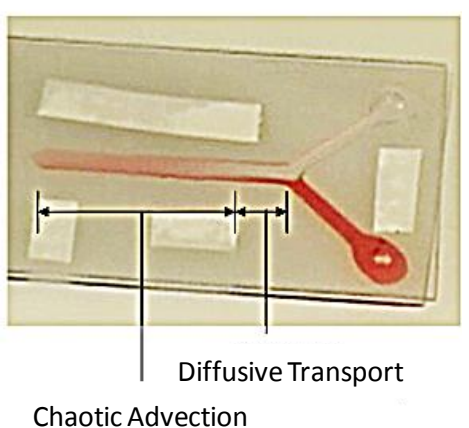

Figure 3.16 Chaotic advection in a surface tension-confined microfluidic platform. (a) Demonstration of platform configuration. (b) Control device without checker pattern diffusive mixing only. (c) Device with checker pattern resulting in chaotic advection and homogenization.

As demonstrated by figure 3.16, there are dual inlets for the two independent fluids to be mixed. In this case, the fluids are water and water with dye in order to preserve similar viscosity and surface tension values for the two fluids. As the fluids are transported through 
the device to the convergence, the streams combine laminarly and a distinct interface is present with strictly diffusive transport across the interface such as in figure $3.16 \mathrm{~b}$. In contrast, when a pattern with abrupt discontinuities in surface energy is presented as illustrated in figure $3.16 \mathrm{a}$ implemented in figure $3.16 \mathrm{c}$, curvature of the fluid interface changes quickly resulting in acceleration and deceleration of adjacent layers of fluid facilitating chaotic advection. As exhibited by figure $3.16 \mathrm{c}$, the stream initially has a distinct interface. As the fluid progresses, the solutions homogenize into the resulting stream.

Since the hydrophilic films do have an associated height $(\sim 1 \mu \mathrm{m}$ as demonstrated in figure 3.8), it is necessary to confirm that the topography of the film is not responsible for the mixing of the fluids within the STCM platform and that the mixing is in fact facilitated by curvature mediation. However, for this study it is necessary to eliminate the curvature-based driving force (i.e. the checked-board pattern) and to see if mixing still occurs. Essentially, in this investigation, the capillary pressure is substituted with a pump-based pressure drop. A three-dimensional chamber is built with surface energy discontinuities and a fluid is pumped over the surface and allowed to evolve to a steady state. For this study, platforms were again patterned using the procedure described in section 3.3.2. The walls of the chamber are made from laser-cutting the polypropylene tape spacer material and properly aligning the spacer with the pattern on the substrate material. Nanoport fittings (Upchurch Scientific) and Teflon tubing are then affixed to inlets drilled in the top substrate. The Teflon tubing is then attached to syringes (BD Falcon) and the syringes are implemented at a flow rate of $0.5 \mathrm{ml} / \mathrm{hour}$ using a syringe pump.

If mixing occurs in the absence of the advancing free surface, then the topography of the film does have an influence on the mixing of the laminar streams. If mixing does not 
occur in the pumped case, then the dynamic velocity of the free surface is responsible for driving the mixing in the STCM platform. Figure 3.17 demonstrates results from this investigation.
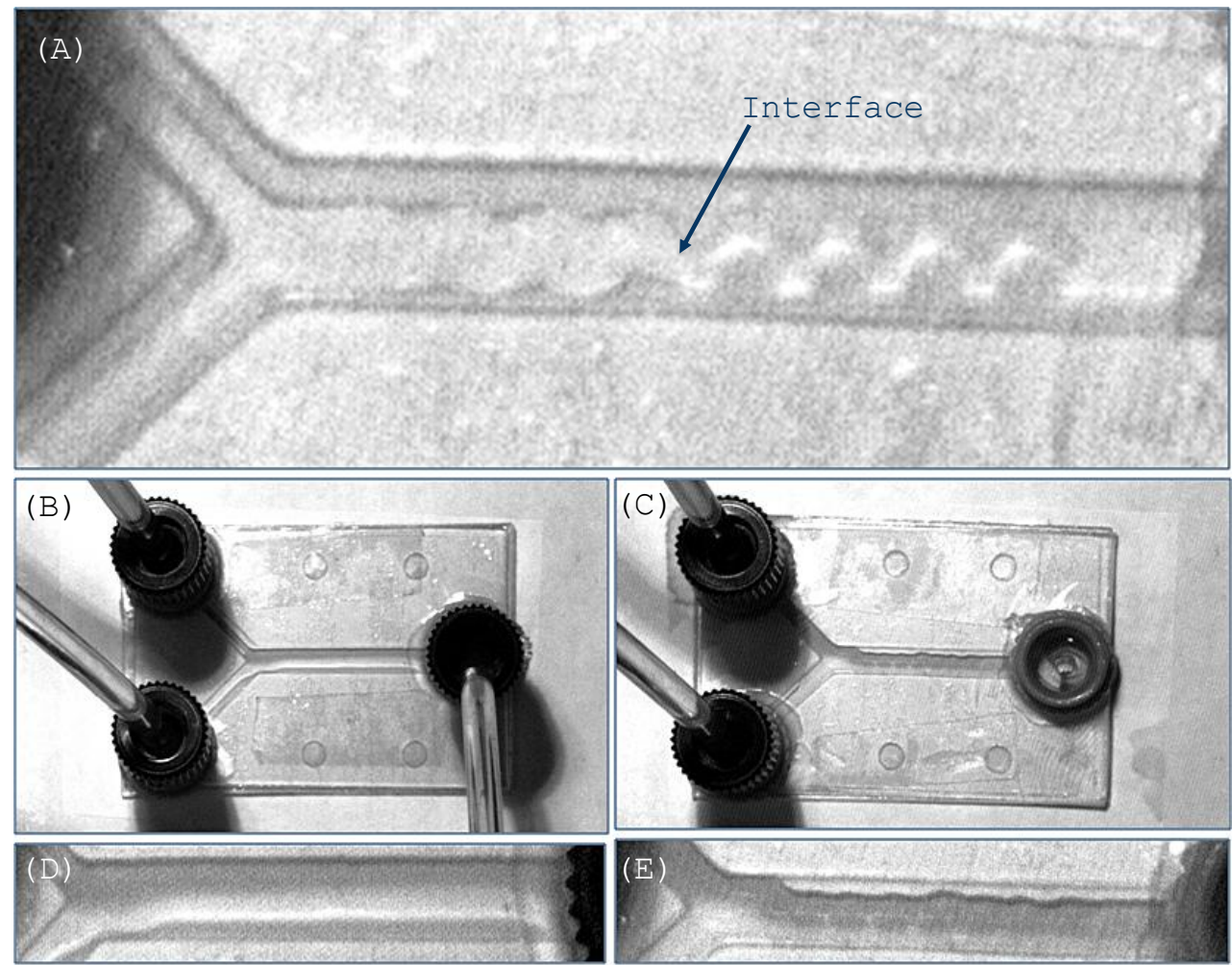

Figure 3.17 Steady-state microfluidic mixer pattern with fluid pumped through the device. (A) Advancing free surface pre-steady state. (B) Control device without checkerboard pattern. (C) Device with checkerboard pattern. (D,E) Zoomed channel of $(\mathbf{B}, \mathbf{C})$, respectively.

As indicated within figure 3.17A, the advancing surface pre-steady state has a preference to stay on the hydrophilic pattern of the in a checkerboard-patterned channel. As the fluid fills the channel and the free surface is no longer present, it becomes apparent in figures $3.17 \mathrm{~B}, 3.17 \mathrm{C}$, that mixing does not occur in either the unpatterned or patterned device. This is further clarified in the zoomed channels of figures 3.17D and 3.17E. Consequently, it becomes apparent that the mixing is, in fact, driven via spatially-dependent dynamic curvature resulting from discontinuities in surface energy. 
Though the STCM passive mixer does not involve sophisticated componentry, the platform is still capable of efficient mixing. Furthermore, the mechanism behind mixing appears to be entirely a result of the surface energy disparities patterned on the substrate surface. As the free surface of an advancing fluid interface passes through the device, and curvature can no longer be manipulated, mixing appears to stop. However, since transport within the chip is achieved via capillarity, the STCM platform is not designed to be operated in a steady-state pumping configuration.

\subsection{Conclusions}

As novel methods are established for the manipulation of micro-volumes of fluids, the amount of invasive or expensive reagents required for chemical or biological analyses is decreased while throughput and parallelization is increased. The automation of such analyses will enable a user with little to no analytical experience to conduct sophisticated laboratory procedures. However, before such technology can be exploited, microfluidic chip processing techniques need to be re-evaluated and optimized in order to make this technology affordable for wide-scale deployment.

In particular, the thick film printing technique is a straight-forward fabrication approach toward developing autonomous microfluidic platforms. As thick film printing is a well-characterized patterning technology, patterning is rather uncomplicated and ultimately scalable into a continuous mass-production scheme. Furthermore, as commodity polymeric materials are utilized, the total cost of platform production is minimal. Subsequently, the thick-film printed microfluidic fabrication approach solves a number of manufacturing dilemmas inhibiting wide-scale deployment of microfluidic 'lab on a chip' technologies. 
In addition to explicating the fabrication approach, theoretical framework has been established enabling intelligent platform design for the integration of chip-based reaction processes. As a result, surface-directed microfluidics is ready to be implemented for novel applications. Such applications are currently under investigation by the author of this chapter.

As inherently simplified as the STCM platform appears to be it is very powerful in device facility. As demonstrated in section 3.3, micro-devices have been fabricated that are capable of enzymatic catalyzed reactions, the determination of protein and glucose presence within a biological sample (and qualitative concentration approximation), and passive mixing through chaotic advection. Consequently, this burgeoning technology promises to mitigate problems associated with mass production of microfluidic total analytical systems.

\subsection{References}

Adamson, A. W. \& Gast, A. P. (1997). Physical Chemistry of Surfaces, 6-thed. WileyInterscience: New York.

Bouaidat, S., Hansen, O., Bruus, H., Berendsen, C., Bau-Madsen, N. K., Thomsen, P., Wolff, A. \& Jonsmann, J. (2005). Lab on a Chip 5, 827-836.

Brakke, K. A. (1992). Experimental Mathematics 1, 141-165.

Brooks, T. \& Keevil, C. W. (1997). Letters in Applied Microbiology 24, 203-206.

Burns, M. A. (1998). Science 282, 484-487.

Butler, J. E., Lue, E. P., Navarro, P. \& Christiansen, B. (1997). Journal of Molecular Recognition 10, 36-51.

Butler, J. E., Navarro, P. \& Lue, P. (1997). Journal of Molecular Recognition 10, 52-62.

Camesasca, M., Kaufman, M. \& Manas-Zloczower, I. (2006a). Journal of Micromechanics and Microengineering 16, 2298-2311.

Camesasca, M., Kaufman, M. \& Manas-Zloczower, I. (2006b). Macromolecular theory and simulations 15, 595-607. 
Camesasca, M., Manas-Zloczower, I. \& Kaufman, M. (2005). Journal of Micromechanics and Microengineering 15, 2038-2044.

Chen, C. F., Kung, C. F., Chen, H. C., Chu, C. C., Chang, C. C. \& Tseng, F. G. (2006). Journal of Micromechanics and Microengineering 16, 1358-1365.

Choban, E. R., Markoski, L. J., Wieckowski, A. \& Kenis, P. J. (2004). Journal of Power Sources 128, 54-60.

Delamarche, E., Bernard, A., Schmid, H., Bietsch, A., Michel, B. \& Biebuyck, H. (1998). J. Am. Chem. Soc 120, 500-508.

Ellerby, L. M., Nishida, C. R., Nishida, F., Yamanaka, S. A., Dunn, B., Valentine, J. S. \& Zink, J. I. (1992). Science 255, 1113.

Eteshola, E. \& Balberg, M. (2004). Biomedical Microdevices 6, 7-9.

Eteshola, E. \& Leckband, D. (2001). Sensors and Actuators B 72, 129-133.

Gill, I. \& Ballesteros, A. (2000). Trends in Biotechnology 18, 282-296.

Goodwin, S. B., Schneider, R. E. \& Fry, W. E. (1995). Plant disease 79, 1181-1185.

Holmes, E. P. J. (1976). Handbook of Thick Film Technology, 2 ed. Bradford, UK: Emerald Group Publishing Limited.

Jayashree, R. S., Gancs, L., Choban, E. R., Primak, A., Natarajan, D., Markoski, L. J. \& Kenis, P. J. A. (2005). J. Am. Chem. Soc 127, 16758-16759.

Johnson, T. J., Ross, D. \& Locascio, L. E. (2002). Anal. Chem 74, 45-51.

Juncker, D., Schmid, H., Drechsler, U., Wolf, H., Wolf, M., Michel, B., de Rooij, N. \& Delamarche, E. (2002). Anal. Chem 74, 6139-6144.

Khandurina, J., McKnight, T. E., Jacobson, S. C., Waters, L. C., Foote, R. S. \& Ramsey, J. M. (2000). Anal. Chem 72, 2995-3000.

Lagally, E. T., Simpson, P. C. \& Mathies, R. A. (2000). Sens. Actuators B 63, 138-146.

Lam, P., Wynne, K. J. \& Wnek, G. E. (2002). Langmuir 18, 948-951.

Leger, L. \& JoannyS, J. F. (1992). Rep. Prog. Phys 431-486.

Mann Jr, J. A. (2002). Journal of dispersion science and technology 23, 199-207. 
Mann Jr, J. A., Romero, L., Rye, R. R. \& Yost, F. G. (1995). Physical Review E 52, 39673972.

Marengo-Rowe, A. J. (1965). J Clin Pathol 18, 790-792.

Martinez, A. W., Phillips, S. T., Butte, M. J. \& Whitesides, G. M. (2007). Angew. Chem. Int. Ed. 46, 1318-1320.

Mastrangelo, C. H., Burns, M. A. \& Burke, D. T. (1998). Proceedings of the IEEE 86, 17691787.

Meera Khan, P. (1971). Arch Biochem Biophys 145, 470-483.

Mitrovski, S. M., Elliott, L. C. C. \& Nuzzo, R. G. (2004). Langmuir 20, 6974-6976.

Nadzhafova, O. Y., Zaitsev, V. N., Drozdova, M. V., Vaze, A. \& Rusling, J. F. (2004). Electrochem. Commun. 6, 205-209.

Nag, A., Panda, B. R. \& Chattopadhyay, A. (2005). Pramana Journal of Physics 65.

Neumann, A. W. \& Good, R. J. (1972). J. Colloid Interface Sci 38, 341-358.

Nguyen, N. T. \& Wu, Z. (2005). J. Micromech. Microeng 15, R1-R16.

Oh, C. S. (1999).

Pugia, M. J., Lott, J. A., Profitt, J. A. \& Cast, T. K. (1999). Journal of Clinical Laboratory Analysis 13, 180-187.

Romero, L. A. \& Yost, F. G. (1996). Journal of Fluid Mechanics 322, 109-129.

Rupcich, N. \& Brennan, J. D. (2003). Analytica Chimica Acta 500, 3-12.

Rye, R. R., Mann, J. J. A. \& Yost, F. G. (1996). Langmuir 12, 555-565.

Rye, R. R., Yost, F. G. \& Mann Jr, J. A. (1996). Langmuir 12, 4625-4627.

Sato, K., Yamanaka, M., Hagino, T., Tokeshi, M., Kimura, H. \& Kitamori, T. (2004). Lab on a Chip 4, 570-575.

Stroock, A. D., Dertinger, S. K. W., Ajdari, A., Mezic, I., Stone, H. A. \& Whitesides, G. M. (2002). Science 295, 647-651.

Stroock, A. D. \& Whitesides, G. M. (2003). Acc. Chem. Res 36, 597-604. 
Suk, J. W. \& Cho, J. H. (2007). Journal of Micromechanics and Microengineering 17, N11N15.

Wang, H., Iovenitti, P., Harvey, E. \& Masood, S. (2003). Journal of Micromechanics and Microengineering 13, 801-808.

Watanabe, M. (2007). Sensors and Actuators B: Chemical 122, 141-147.

West, J., Michels, A., Kittel, S., Jacob, P. \& Franzke, J. (2007). Lab Chip 7, 981-983.

White, N. M. \& Turner, J. D. (1997). Meas. Sci. Technol 8.

Xia, Y. \& Whitesides, G. M. (1998). Angew. Chemie Int. Ed. 37, 550-575.

Zhao, B., Moore, J. S. \& Beebe, D. J. (2001). Science 291, 1023-1026.

Zhao, B., Moore, J. S. \& Beebe, D. J. (2002). Anal. Chem 74, 4259-4268.

Zhmud, B. V., Tiberg, F. \& Hallstensson, K. (2000). Journal of Colloid and Interface Science 228, 263-269. 


\section{Chapter 4: Alternative Methodology for Platform}

\section{Fabrication}

\subsection{Limitations of current fabrication techniques}

Although a consistent fabrication procedure capable of producing platforms capable of autonomous fluid transport is developed, the approach is not without limitations. Specifically, since the manufacturing strategy entails patterning materials of drastically opposing molecular properties, adhesion is a great concern. Following the proper fabrication protocols expounded in chapter 3, platforms can be consistently produced. However, the adhesion of the hydrophilic conduit material (i.e. cellulose acetate) is poor on the resulting substrate.

Once the device is configured, the paths are sufficiently protected from delamination. Nevertheless, during processing, devices can be damaged and rendered useless if great care is not taken to ensure the platform surface is not damaged. Accordingly, several alternative fabrication protocols are under investigation.

\subsection{Thick film printing of materials with intermediate wettability}

An attractive material for capillary transport initially considered for device manufacture is polyvinyl alcohol. Due to the high degree of hydroxylation of this material, hydrogen bonding is possible and the resulting film is rather wettable. Furthermore, the fullyhydrolyzed polymer remains substantially hydrophilic while maintaining the ability to avoid dissolution as opposed to other hydrophilic polymers such as poly(ethylene glycol) and 
polyvinyl pyrrolidone. The disadvantage of this polyvinyl alcohol is that there are only a limited number of solvents that can suitably dissolve this material. Furthermore, these solvents tend to be too volatile for implementation through screen printing resulting in the clogging of screens and the inability to print. In addition, the hydroxyl groups on the polyvinyl alcohol backbone can hydrogen bond amongst adjacent chains resulting in intermolecular cross-linking and gelation of the polymer ink.

A possible means to circumvent the problems associated with the direct printing of polyvinyl alcohol includes printing precursor materials of intermediate wettability. Subsequently, by performing simple chemistry, polyvinyl alcohol can be regenerated resulting in substantial surface wettability as well as preserving adequate adhesion to the hydrophobic substrate.

Before this approach can be exploited, it is necessary to understand the production of polyvinyl alcohol (PVA) and the further derivitization thereof. First, vinyl acetate is polymerized to polyvinyl acetate (PVAc). Methods to achieve an efficient polymerization and purification of polyvinyl acetate are robust and numerous (Tanner, 1959, Matsumoto \& Imai, 1965, John, 1969, John \& Edward, 1958, Bristol, 1952). The resulting polyvinyl acetate is subsequently hydrolyzed to polyvinyl alcohol via the introduction of either strong acid or base (Wu, 1989). The polyvinyl alcohol can be converted to polyvinyl formal (PVF) through reaction with formaldehyde or analogously to polyvinyl butyral (PVB) through reaction with butyraldehyde (Martien, 1987). Moreover, the conversion to polyvinyl formal and polyvinyl butyral are reversible via the addition of strong acid or base (Ma et al., 2007). The formation and derivitization processes are summarized in figure 4.1. 


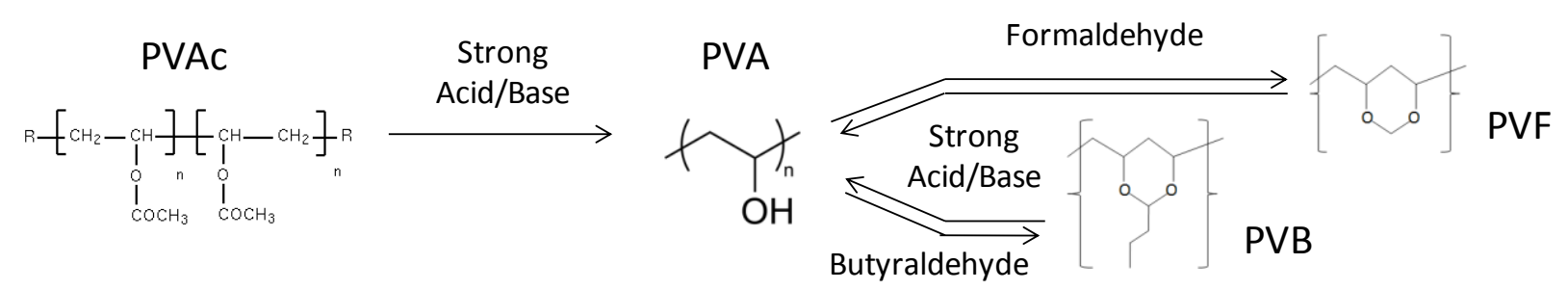

Figure 4.1 Formation and derivitization of polyvinyl alcohol.

Having knowledge of the production and derivitization of polyvinyl alcohol enables the strategic patterning and subsequent modification of the vinyl polymers. The concerns of direct patterning of polyvinyl alcohol are mitigated by patterning the vinyl polymers and reacting the resulting films with strong acid/base to regenerate polyvinyl alcohol. All of the vinyl polymers are soluble in the low volatility solvent tetraethylene glycol dimethyl ether enabling patterning without screen clogging. Furthermore, the solutions are either not capable of gelation or less capable in contrast to the polyvinyl alcohol solution. In addition, because the vinyl polymers have a greater degree of hydrophobic character than polyvinyl alcohol, adhesion is improved substantially through the indirect route of producing polyvinyl alcohol.

Solutions of the vinyl polymers are prepared at various weight percentages. The polyvinyl formal still suffers from the capacity to form gels inhibiting patterning. The polyvinyl acetate and polyvinyl butyral both pattern rather well on the polyvinyl chloride substrate materials utilizing the MPM TF-100 thick film printer. Hydrolization is attempted on the subsequent films through a timed soak in $10 \% \mathrm{NaOH}$ (aqueous), $10 \% \mathrm{KOH}$ (in methanol) or $1.0 \mathrm{M} \mathrm{HCl}$. Figure 4.2 demonstrates the results from a study of the kinetics of acidic hydrolysis of PVB through the measurement of contact angle using a Ramé Hart goniometer implementing the sessile drop contact angle method. As illustrated in figure 4.2, 
the contact angle of the PVB drops form an initial value of $76.9^{\circ} \pm 1.6^{\circ}$ to a value of $68.2^{\circ} \pm 0.4^{\circ}$ over a 24 hour period. Although this is a significant drop in contact angle, it still is not competitive with the cellulose acetate material (contact angle $=60.0^{\circ} \pm 0.3^{\circ}$ ) in supporting autonomous fluid transport. In contrast to the PVB material, the PVAc material is soaked in $1.0 \mathrm{M} \mathrm{HCl}$ for 18 hours resulting in contact angle change from $72.0^{\circ} \pm 1.6^{\circ}$ to $69.7^{\circ} \pm 2.2^{\circ}$ which is not significant enough to warrant the additional step of handling caustic materials. Similar results are attained for the alternative hydrolysis solutions using $10 \%$ $\mathrm{NaOH}$ and $10 \% \mathrm{KOH}$. Additionally, the acid/base treatment was attempted on cellulose acetate in an effort to regenerate cellulose without a substantial improvement in wettability.

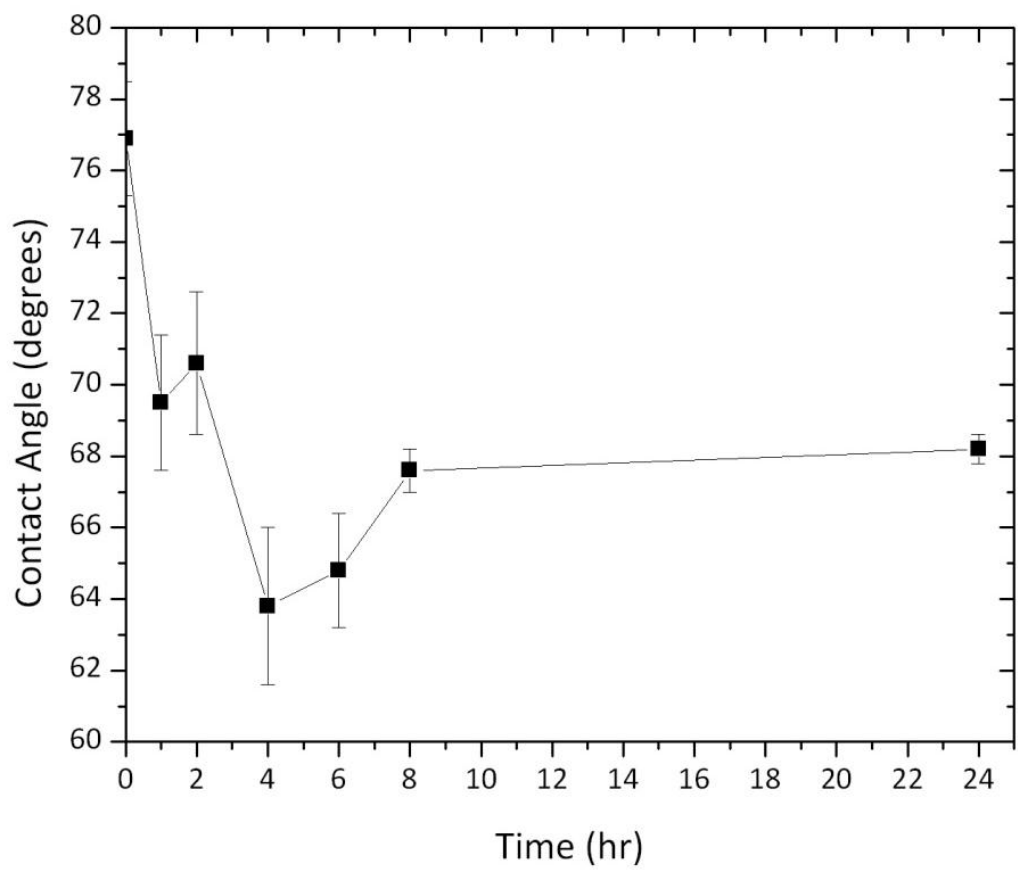

Figure 4.2 Kinetics of hydrolysis of PVB in 1.0 M HCl through contact angle measurement.

Although the technique of patterning vinyl polymers and attempting surface chemistry to produce a layer of polyvinyl alcohol alleviates many problems associated with 
the direct printing of polyvinyl alcohol, at the stage of the investigation, there is no evidence supporting that the additional step requiring caustic substances is an improvement on the function of the surface tension-confined microfluidic devices fabricated in chapter 3 . However, the general approach of patterning a material of intermediate wettability and the subsequent chemical modification of the resulting film could potentially be applied to other materials with greater success than the materials investigated in this study.

\subsection{Microplasma writing of hydrophilic paths upon polymeric substrates}

Plasma chemistry presents a powerful means for modifying a variety of properties. Several advantages include: (1) Modification can be confined strictly to the surface without altering bulk properties (2) plasmas are highly reactive (and consequently non-specific) affording the ability to perform modification on surfaces regardless of chemical structure (3) modification can be easily tailored for specific purposes by choosing the appropriate gas source (4) the lack of wet chemicals eliminates problems associated polymer swelling/dissolution and subsequent solvent removal steps and (5) although the modification is non-specific it is macroscopically uniform (Chan et al., 1996). Disadvantages of traditional plasma processing include that the procedure must occur in a reactor drawn to a vacuum where the entire material surface is indiscriminately affected. As a result, this process requires load/unload procedures rendering it an inherently batch technique.

In contrast, plasma systems operating at ambient pressure have been developed and thorough literature exists regarding their operation (Schutze et al., 1998). In addition, techniques exist to produce microplasma beams in ambient conditions enabling the ability to pattern a surface without complicated masking techniques (Cho et al., 2001). This 
technology has been applied to pattern holes and lines on silicon wafers (Sankaran, 2001), create radical species for thin film deposition (Sankaran, 2002), etch 2D and 3D microstructures and produces substrates capable of facilitating surface-enhanced Raman spectroscopy (Chiang et al., 2007).

Furthermore, plasma treatments in general can be utilized to perform surface chemistry capable of altering wettability. This has been successfully demonstrated for polymers including poly(tetrafluoro ethylene) (Morra et al., 1989, Wilson et al., 2000), poly(ethylene terephthalate) (Beake et al., 1998, Paynter, 2000), and polystyrene (Beake et al., 1998, Paynter, 2000) to name a few. As a result, controlled microplasma treatment provides a viable means to control surface contact angle.

In collaboration with Professor Mohan Sankaran and Matt Cochey, an atmosphericpressure microplasma is utilized to investigate altering the contact angle of a poly(methyl methacrylate) (PMMA) surface. The microplasma system is composed of a stainless steel capillary tube $(\mathrm{ID}=250 \mu \mathrm{m})$ attached to a DC power supply and a gas source with a stepping motor in the $z$-direction. The substrate is placed on a stage with numerically-driven stepping motors in the $x, y$-directions. Pure argon gas is fed to the system at a flow rate of 200 standard cubic centimeters per minute. A series of lines are patterned upon a substrate in a serpentine pattern at a scan rate of $6 \mathrm{~mm} / \mathrm{s}$. The lines are sufficiently close to one another that a square of surface is modified (10 vertical lines are patterned at a spacing of $500 \mu \mathrm{m})$. The experimental setup is depicted by figure 4.3 . 


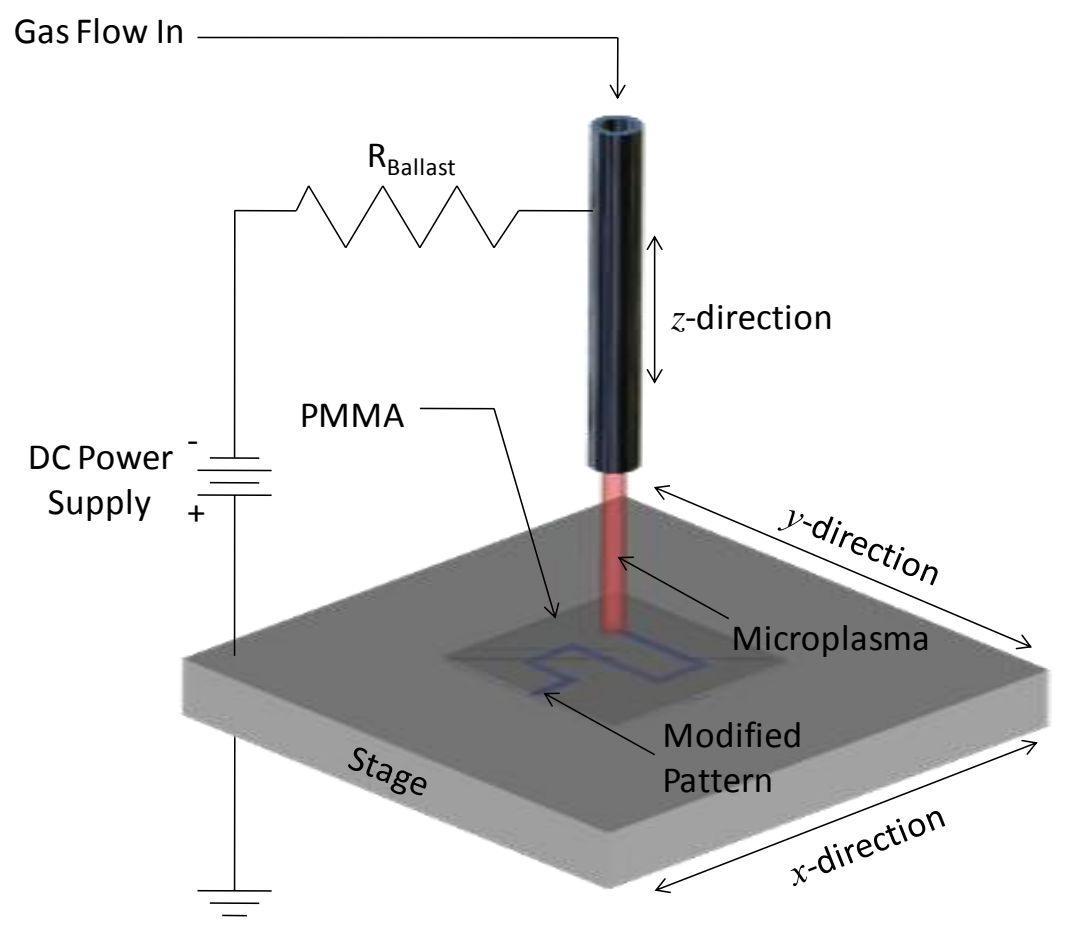

Figure 4.3 Demonstration of microplasma setup (adapted from Chiang et al., 2007).

Contact angle measurements are performed to determine the effectiveness of the microplasma patterning technique. The sessile drop method is utilized and with a Ramé Hart goniometer. An initial contact angle of $73.0^{\circ} \pm 3.0^{\circ}$ is measured for the untreated PMMA surface. Following patterning, the contact angle drops substantially to a value of $15.8^{\circ} \pm 2.8^{\circ}$. However, as aging occurs, the contact angle begins to increase as the polymer surface begins to reorganize through ambient storage. Figure 4.4 depicts the aging trend and loss of wettability over time. 


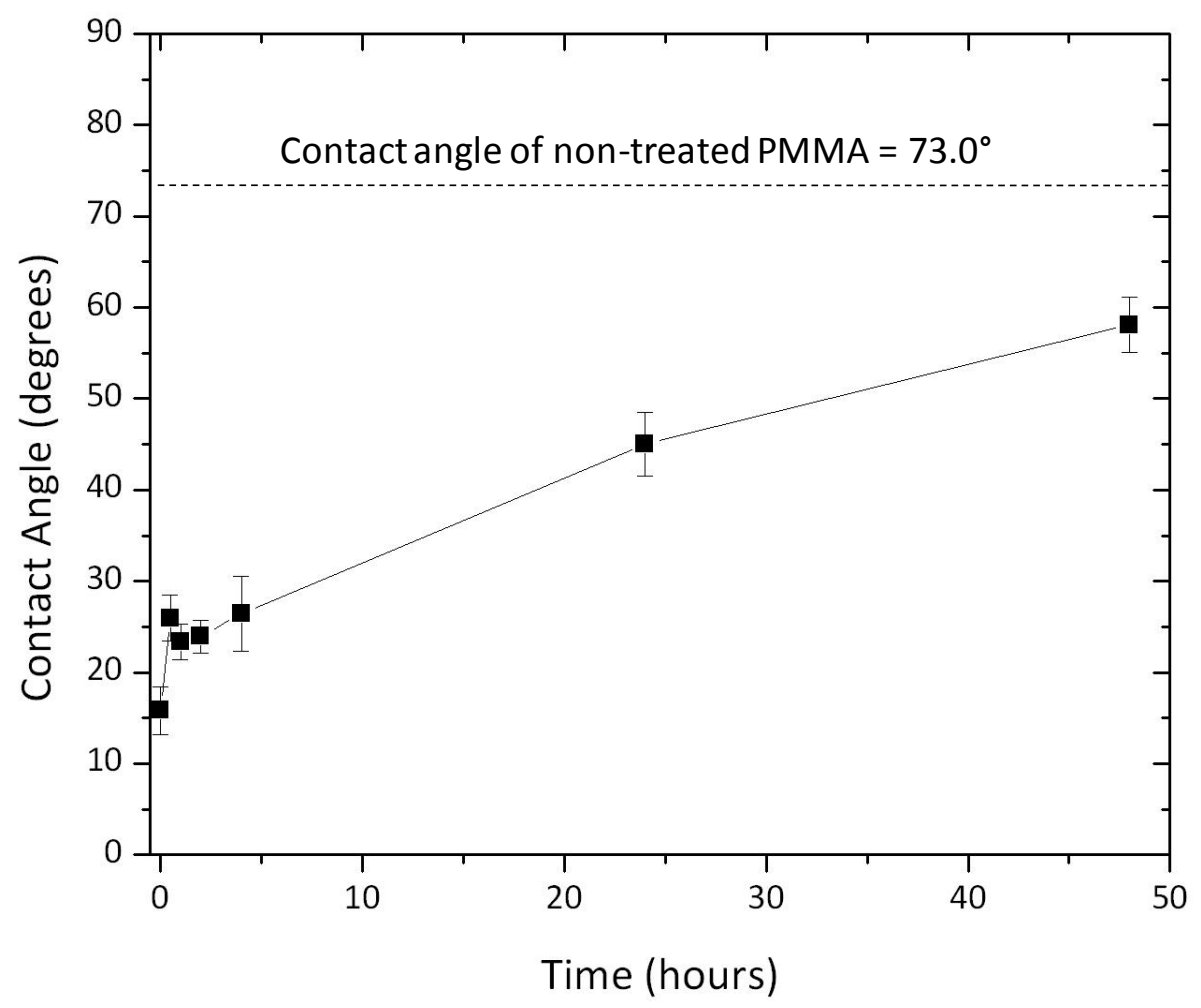

Figure 4.4 Contact angle over a period of time for microplasma-patterned PMMA.

The microplasma patterning technique provides a useful means to manipulate surface wettability. Furthermore, this technique mitigates problems associated with traditional plasma processing as it can be performed under ambient pressure and does not require load/unload procedures. Consequently, the microplasma patterning approach is suitable for roll-to-roll processing. However, due to the reorganization of the polymer surface, almost all wettability is lost within a matter of 48 hours. The challenge of inhibiting reorganization may be resolved by investigating alternative packaging or storing of patterned devices. Since air is largely composed of non-polar molecules, reorganization tends to occur favoring non-polar functionalities to be brought to the polymer surface. Consequently, it is speculated that storage in either vacuum or a polar media (e.g. storage in under an atmosphere of ammonia or storage in water) may prove to maintain the wettability of the patterned surface. 
Optimization of experimental conditions is still in progress and other hydrophobic substrate material is slated to commence soon. An investigation in proper storage will begin when the patterning technique is optimized.

\subsection{Ultraviolet light-mediated path formation}

An alternative approach to patterning hydrophilic regions upon an otherwise hydrophobic substrate worthy of investigation entails light-mediated surface modification using ultraviolet light. The successful development of such techniques would enable the use of photomasks for patterning affording the ability to drastically decrease path widths for operation within the nanofluidics regime. Two approaches of interest involve photoacid generation for surface hydrolysis and the UV-initiated photografting of hydrophilic molecules upon polyethylene. Preliminary data exists for these techniques although the protocols are currently not optimized.

\subsubsection{Photoacid generators for side-group hydrolysis}

A critical component for photoresist materials enabling ultraviolet (UV) light catalyzed deprotection include photoacid generators. Photoacid generators are typically benign under light from the visible region and can be directly integrated into a resist film. Upon exposure to UV light, the acid becomes activated resulting in hydrolysis of acrylic side

groups on a polymer backbone (Lamanna et al., 2002). Subsequently, the resulting reacted region of the film may become water soluble and enables the dissolution of this region leaving a distinct pattern within the non-hydrolyzed film. 
This approach to hydrolyzing a 3D matrix of polymer and photoacid generator has a 2D analog for the chemical modification of a polymer surface. The concept is that a solution of photoacid generator can be placed on the surface of a polymer with groups subject to hydrolysis (e.g. acetate or acrylate groups - polymers with ether linkage). Next a photomask can be aligned above the solution and a UV lamp can initiate acidification of the photoacid generator. The resulting acid will then facilitate chemistry cleaving side groups thereby affecting the wettability of the resulting region unmasked by the photomask.

This process is dependent upon choosing a material that is sufficiently hydrophobic prior to hydrolysis while leaving a substantially more hydrophilic region after hydrolysis. For this reason, hydrolysis of an acrylic group resulting in replacement with a carboxylic acid is very attractive (see figure 4.5). An initial option that quickly comes to mind would be poly(methyl methacrylate) since it this acrylic material is widely available and inexpensive $\left(\mathrm{R}_{1}\right.$ and $\mathrm{R}_{2}$ are both $\left.-\mathrm{CH}_{3}\right)$. However, after hydrolysis and cleavage of the methacrylate group, the resulting surface still has a hydrophobic methyl group on the carbon backbone. Consequently, other materials are under consideration that better fulfill the initial criteria of a drastic change in surface energy upon hydrolysis. Currently, poly(ethyl acrylate) (PEA) is under investigation $\left(\mathrm{R}_{1}\right.$ is $-\mathrm{CH}_{2} \mathrm{CH}_{3}$ and $\mathrm{R}_{2}$ is $\left.-\mathrm{H}\right)$.

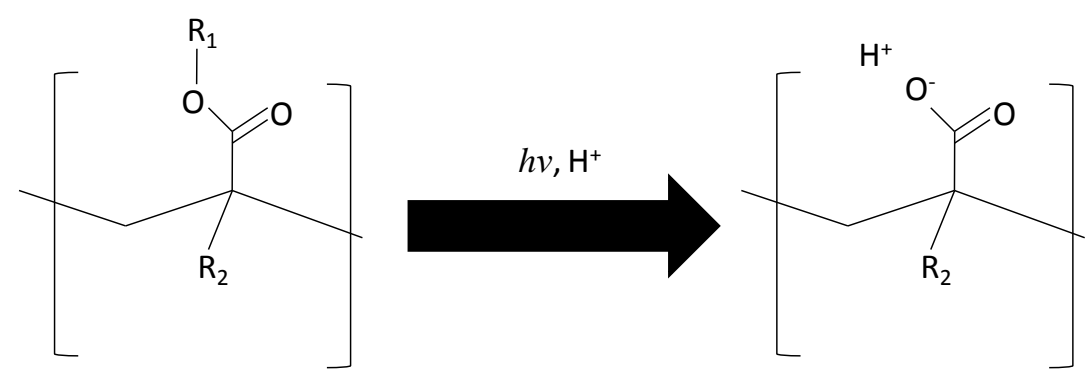

Figure 4.5 Schematic of hydrolysis of an acrylic polymer enabling wettability mediation. 
Films of poly(ethacrylate) are prepared through solvent casting of a $20 \%(\mathrm{w} / \mathrm{w})$ solution of PEA dissolved in toluene (Sigma-Aldrich; Milwaukee, WI) upon a glass microscope slide substrate. The toluene is allowed to evaporate overnight. The following day, a 1.0 M solution of $\mathrm{HCl}$ is placed on the film surface. The $\mathrm{HCl}$ is again allowed to evaporate and an increase in wettability is initially apparent when washing the film with distilled water. However, as the film is washed over time, the wettability appears to diminish. Furthermore, as a droplet of water is placed on the hydrolyzed film, the droplet does not appear to spread anymore than a droplet on the untreated hydrophobic region. As time passes, the droplet becomes cloudy on the hydrolyzed region while a droplet remains transparent on the untreated areas. These initial experiments seem to indicate that the resulting film, effectively poly(acrylic acid), is water soluble. This result is not a surprise; however, the hope was that chain entanglement with the water insoluble PEA would fix the poly(acrylic acid) layer onto the surface of the film.

Although this investigation is still under consideration, the proof of concept that acrylic polymer surfaces can be hydrolyzed to a more hydrophilic state with strong acid has been established. The next steps include exploring other polymer or copolymer systems that result in a water insoluble layer that is sufficiently hydrophilic and then the extension to using a suitable photoacid generator and photomask for the production of STCM prototypes.

\subsubsection{Aliphatic ketones as photoinitiators}

Polyethylene is a sufficiently hydrophobic and an inexpensive material that could potentially be used for a substrate material. However, since polyethylene is resistant to 
dissolution in a wide variety of solvents, attaining adhesion of a film of hydrophilic material on a polyethylene substrate is a challenge (i.e. substrate etching with a co-solvent cannot be used to facilitate chain entanglement for increased adhesion). A possible means to circumvent designing an appropriate solvent system for thick film printing includes the direct chemical modification of the polyethylene via photografting.

Due to the minimal cost of polyethylene, it is often an attractive substrate for plastic materials. However, the inherent chemical inertness of polyethylene warrants surface modification to improve properties such as wettability. This can be achieved through hydrogen abstraction with a radical species in the presence of a material with an allyl bond. Photo-initiation of radicals to alter the properties of a polyethylene substrate is not necessarily a new technique. Photografting can be achieved through a vapor phase reaction (Allmear et al., 1988, Lei et al., 2000) or in the liquid phase (Kubota \& Koyama, 1997, Ogiwara et al., 1988).

As of recent, photografting procedures are becoming substantially simplified. For example, Wang, et al., have demonstrated that photografting of methacrylic acid can be efficiently achieved through photodecomposition of aliphatic ketones resulting in the abstraction of a hydrogen from the polyethylene surface and yielding the self-initiated grafting of methacrylic acid. These results are extended upon in the current investigation in an effort to modulate polyethylene wettability utilizing acrylic acid.

A proposed mechanism for this process can be adapted from previously reported results (Allmer et al., 1988, Wang et al., 2007). According to Allmer, et al., after absorbing ultraviolet light, 2-butanone can split in a Norrish type 1 reaction leaving acetyl, methyl and ethyl radicals or concomitantly the 2-butanone can abstract a hydrogen from the polymer 
surface resulting in a radical (see figure 4.6a). The polymer radical can thereby self-initiate the grafting of the acrylic acid increasing the wettability of the surface (figure 4.6b). The other radical species in the reaction can either combine to form inactive products or cause homo-polymerization of the acrylic acid in the liquid phase.

(a)

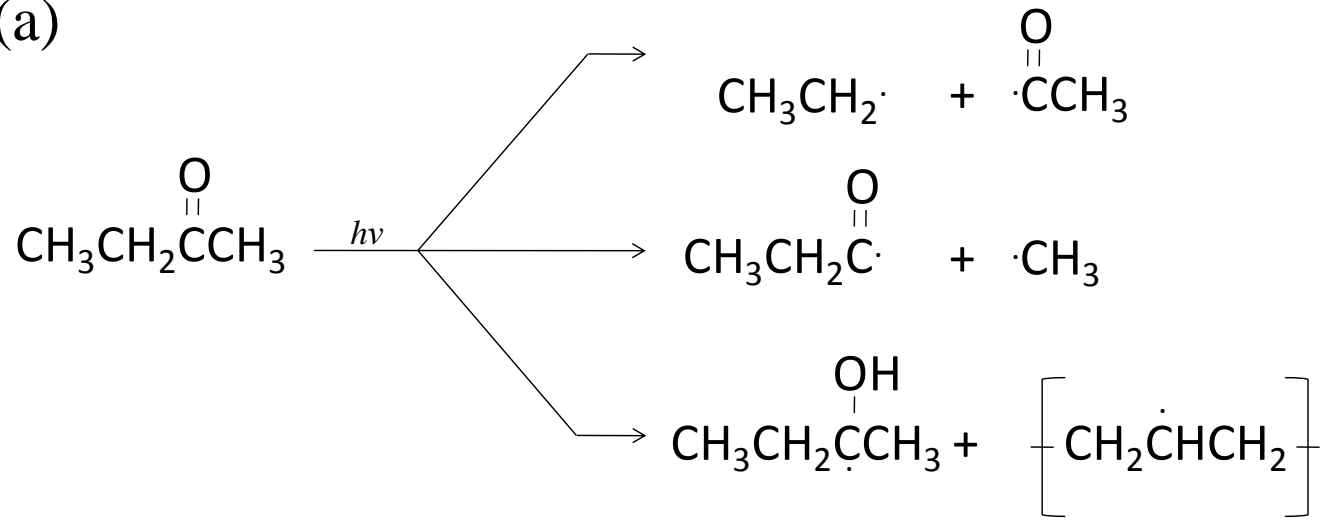

(b)

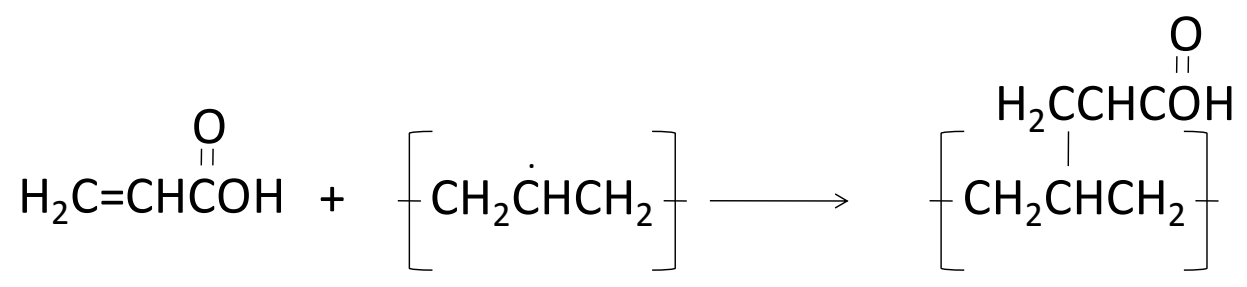

Figure 4.6 Proposed mechanism for the (a) photodecomposition of 2-butanone and (b) subsequent self-initiation of acrylic acid grafting to HDPE surface.

High-density polyethylene (HDPE) is utilized (Dow Chemical Company) as a substrate material. The HDPE pellets are heated above $115{ }^{\circ} \mathrm{C}$ and pressed between glass slides resulting in a thin substrate following temperature quench. The HDPE substrate is then placed in acetone bath overnight for the removal of surface contaminants, residual monomer or other polymerization materials.

A reaction solution is prepared of $10 \%(\mathrm{w} / \mathrm{w})$ acrylic acid, $10 \%(\mathrm{w} / \mathrm{w})$ 2-butanone and $80 \%(\mathrm{w} / \mathrm{w})$ distilled water. The HDPE film is then put in the reaction solution and radical 
formation is initiated with an Oriel Xenon Lamp (model 66002) powered by an Oriel 68805 Universal Power Supply. The reaction is allowed to proceed for 5 minutes. Following the grafting procedures, a contact angle change from $92.6^{\circ} \pm 1.2^{\circ}$ to $68.4^{\circ} \pm 3.9^{\circ}$ is observed.

Although the current procedure is still non-optimized, a substantial increase in wettability of the grafted-substrate is achieved. The overall process requires inexpensive materials and reagents. Furthermore, attempts have been made to manufacture microfluidic patterns using photomasks produced in house. However, only a limited amount of success has been attained to date due to problems with the orientation of the xenon lamp and the inadequacy of photomasks produced with a typical office laserjet printer (HP 1200 Laserjet). The implication of optimizing this technique is that it would enable a much greater resolution to be attained with state of the art photomasks than in regard to thick film printing (i.e. on the order of hundreds of nanometers rather than hundreds of microns).

\subsection{Concluding Remarks and Suggestions}

As discussed during the outset of this chapter, the thick film printing process is consistent and can produce autonomous microfluidic platforms with a high degree of reliability. Nevertheless, the resulting platforms are subject to poor path adhesion and can be damaged if not properly and quickly assembled. In addition, the resolution that can be attained with the screen printing process is approximately 300 micrometers. Consequently, there are aspects that can be improved with regards to platform ruggedness and attainable path resolution.

A possible solution to the poor adhesion of chemically dissimilar materials is to print a material of intermediate surface energetics and perform subsequent chemistry to render the 
surface sufficiently hydrophilic. This is demonstrated via the thick film printing of polyvinyl acetate and polyvinyl butyral along a subsequent hydrolysis step regenerating a surface layer of polyvinyl alcohol. Although this technique is successful in improving adhesion, the resulting surface was not as hydrophilic as what can be attained through directly printing cellulose acetate and using the device as is.

Furthermore, more exotic techniques are currently under investigation not involving thick film printing at all. For example, using an atmospheric microplasma along with a stage and numerically-controlled stepping motors, it is possible to directly pattern a polymer surface and attain substantial contact angle change. The disadvantage of this approach is that the polymer surface will begin to reorganize in the presence of air and the pattern is lost over time. As a result, an investigation on suitable storage processes maintaining contact angle is of interest.

In addition to microplasma patterning, UV mediated patterning through a photomask may be possible. Initial studies indicate that strong acids are capable of the hydrolysis of acrylic polymers resulting in the formation of a carboxylic acid surface. These results indicate that photoacid generators may present the opportunity to pattern the surface of an acrylic polymer. In addition, photografting of acrylic acid onto polyethylene is demonstrated to result in a substantial change in wettability of the resulting film. The UV modulated techniques are currently non-optimized and need to be investigated further before they become reasonable alternatives to the thick film printing process. 


\subsection{References}

Allmer, K., Hult, A. \& Ranby, B. (1988). Journal of Polymer Science Part A Polymer Chemistry 26, 2099-2111.

Beake, B. D., Ling, J. S. G. \& Leggett, G. J. (1998). J. Mater. Chem 8, 2845-2854.

Bristol, J. (1952). United States Patent Office 2,614,088.

Chan, C. M., Ko, T. M. \& Hiraoka, H. (1996). Surface science reports 24, 1-54.

Chiang, W. H., Cochey, M., Virnelson, R. C. \& Sankaran, R. M. (2007). Applied Physics Letters 91, 021501.

Cho, B. O., Hwang, S. W., Lee, G. R. \& Moon, S. H. (2001). Journal of Vacuum Science \& Technology A: Vacuum, Surfaces, and Films 19, 730.

John, E. \& Edward, P. C. (1958). Canadian Intellectual Property Office CA 565,080.

John, J. H. (1969). Canadian Intellectual Property Office CA 815,109.

Kubota, H. \& Koyama, M. (1997). Journal of applied polymer science 63, 1635-1641.

Lamanna, W. M., Kessel, C. R., Savu, P. M., Cheburkov, Y., Brinduse, S., Kestner, T. A., Lillquist, G. J., Parent, M. J., Moorhouse, K. S. \& Zhang, Y. (2002). Proceedings SPIE The International Society for Optical Engineering 817-828.

Lei, J., Gao, J., Zhou, R., Zhang, B. \& Wang, J. (2000). Polymer International 49, 14921495.

Ma, X., Sun, Q., Su, Y., Wang, Y. \& Jiang, Z. (2007). Separation and Purification Technology 54, 220-226.

Martien, F. L. (1987). Vinyl alcohol polymers, Vol. 17, Encyclopedia of Polymer Science and Engineering

edited by H. F. Mark, N. M. Bikales, C. G. Overberger, G. Menges \& J. I. Kroschwitz, New York: Wiley-Interscience.

Matsumoto, M. \& Imai, K. (1965). United State Patent Office 3,211,712.

Morra, M., Occhiello, E. \& Garbassi, F. (1989). Langmuir 5, 872-876. 
Ogiwara, Y., Koike, N., Kubota, H. \& Hata, Y. (1988). Journal of applied polymer science $35,1473-1481$.

Paynter, R. W. (2000). Surface and Interface Analysis 29, 56-64.

Sankaran, R. M. (2001). Applied Physics Letters 79, 593.

Sankaran, R. M. (2002). Journal of Applied Physics 92, 2406.

Schutze, A., Jeong, J. Y., Babayan, S. E., Park, J., Selwyn, G. S. \& Hicks, R. F. (1998). IEEE Transactions on Plasma Science 26, 1685-1694.

Tanner, W. B. (1959). United States Patent Office 2,878,168.

Wang, H., Brown, H. R. \& Li, Z. (2007). Polymer 48, 939-948.

Wilson, D. J., Eccles, A. J., Steele, T. A., Williams, R. L. \& Pond, R. C. (2000). Surface and Interface Analysis 30, 36-39.

Wu, T. C. (1989). United States Patent Office 4,820,767. 


\section{Chapter 5: Future of Surface Tension-Confined Microfluidics}

\subsection{Optimizing platforms made via thick film printing}

Currently, a robust thick film printing process is established resulting in the consistent manufacture of autonomous surface tension-confined microfluidic platforms. The fabrication process requires minimal materials, is quick to perform, can be implemented continuously and is reliable. However, there are problems associated with film adhesion and resolution that warrant further investigation for the possibility of improvement. The first section of chapter 5 will address the potential areas of improvement for the surface tension-confined microfluidic (STCM) platform.

\subsubsection{Suitable material systems}

To date, several materials were investigated for hydrophilic conduits and hydrophobic substrates. The nature of trying to attain adhesion of such chemically dissimilar materials immediately renders this a challenging endeavor. Consequently, a wide variety of materials are examined for device development. Table 5.1 summarizes a variety of materials currently investigated along with a comment regarding the efficacy of the material for producing consistently functioning devices. 
Table 5.1 Materials investigated for the production of STCM platforms.

\begin{tabular}{clc}
\hline Material & Purpose & Comment \\
\hline ES-40 Silica sol gel & Hydrophilic Conduit & Mechanically rigid - may crack and delaminate \\
ES-50 Silica sol gel & Hydrophilic Conduit & Mechanically rigid - may crack and delaminate \\
MS-57 Silica sol gel & Hydrophilic Conduit & Mechanically rigid - may crack and delaminate \\
Cellulose Acetate & Hydrophilic Conduit & Suitable material - subject to delamination \\
Poly(methyl methacrylate) & Hydrophilic Conduit & Not hydrophilic enough to produce confined flow \\
Polycarbonate & Hydrophilic Conduit & Not hydrophilic enough to produce confined flow \\
Polyvinyl acetate & Hydrophilic Conduit & Not hydrophilic enough to produce confined flow \\
Polyvinyl alcohol & Hydrophilic Conduit & Many problems associated with patterning \\
Poly(methyl methacrylate) & Hydrophobic Substrate & Cannot maintain confinement \\
Polycarbonate & Hydrophobic Substrate & Cannot maintain confinement \\
Polystyrene & Hydrophobic Substrate & Works well but tedious to process substrates \\
Rinzl PVC & Hydrophobic Substrate & Works well but delamination possible \\
Polyethylene & Hydrophobic Substrate & Cannot achieve adhesion \\
Polypropylene & Hydrophobic Substrate & Cannot achieve adhesion \\
Poly(tetrafluoro ethylene) & Hydrophobic Substrate & Cannot achieve adhesion
\end{tabular}

Several routes to produce silica sol gel are considered along with an in depth protocol facilitating the formation of a glass matrix discussed in section 3.2.1. The precursor materials include poly(diethoxysiloxane) (ES) and poly(dimethoxysiloxane) (MS) of $\mathrm{SiO}_{2}$ equivalencies of $40 \%, 50 \%$ and $57 \%$ (Gelest; Morrisville, PA). Several devices were patterned via pen plotting. However, after patterning and gel syneresis upon polymerization, the paths begin to crack and delaminate. The high $\mathrm{SiO}_{2}$ equivalency MS-57 gel appears to undergo the least syneresis and produce the most consistent devices. This approach was ultimately deemed not worthy of further investigation as the HP7475A pen plotter is not a consistent fabrication technology. However, the sol gel chemistry does produce substantially hydrophilic films. Perhaps the development of a patterning technique able to process low viscosity solutions would increase the efficacy of the sol gel route.

Cellulose acetate and polyvinyl acetate print rather well. However, polyvinyl acetate lacks the necessary hydrophilicity to produce autonomous flow. Polyvinyl alcohol is also considered but due to problems with finding an appropriate solvent system and the gelation 
of this material when in solvent, this material is non-optimal. Stencil patterning was considered for polyvinyl alcohol patterning; however, stencil manufacturing techniques at best enable a resolution of millimeters rather than microns. Cellulose acetate seems to be the best material to date.

Due to the intermediate wettability of poly(methyl methacrylate) and polycarbonate, these materials are considered for both hydrophilic conduit and hydrophobic substrate. As hydrophobic substrate materials, neither material is capable of confined flow. As hydrophilic conduit, confined flow is achieved but at a staggeringly slow flow rate.

Polystyrene and Rinzl polyvinyl chloride (PVC) function rather well as hydrophobic substrates. The PVC material is very convenient to work with since it can be easily processed to size and is mechanically pliable enough not to crack or fracture under stress. The polystyrene material functions well and also has the added benefit of being susceptible to etching with a wide variety of solvents enabling solvent welding and chain entanglement for the production of monolithic platforms. Nevertheless, since polystyrene is brittle, processing this material to an appropriate size pre-patterning is rather tedious. The development of polystyrene processing techniques might make this material more attractive (e.g. the development of an injection molding system for substrate preparation)

With regard to thick film printing, polyethylene, polypropylene and poly(tetrafluoro ethylene) are far too hydrophobic and chemically inert to achieve sufficient adhesion. In addition to adhesion problems, shortly after patterning the hydrophilic ink solution, dewetting can occur and create a 'beading' of the ink resulting in loss of pattern. Lastly, the optical properties of these materials is non-optimal. 
As a consequence of this discussion, the best material system investigated for thick film patterning remains cellulose acetate hydrophilic conduits upon the hydrophobic polyvinyl chloride substrates. This material system results in the successful production of STCM platforms. Ultimately, there is still room for improvement for device production.

\subsubsection{New materials to explore}

Although a plethora of materials have been considered, there is still opportunity to continue and investigate new material systems. A wide variety of commodity polymers have already been integrated into a surface tension-confined microfluidic platform. However, there are still many materials that can be considered.

Several water-soluble materials might provide the opportunity to guide fluid flow. This technique has been demonstrated as a viable means to produce microfluidic devices (Watanabe, 2007). The most suitable material investigated by Watanabe includes 2-(2ethoxyethoxy)ethanol. In this study, water-soluble materials are inkjet-printed onto sufficiently hydrophobic chemically-modified glass. Potentially, this approach can be extended and a water-soluble material may potentially provide improved device function. For example, polyvinyl alcohol, poly(ethylene glycol) or poly(vinylpyrrolidinone) could be ink jet printed upon a hydrophobic substrate for the development of guided flow.

In addition to readily water-soluble path materials, fully-hydrolyzed polyvinyl alcohol may provide an improvement on function pending a suitable patterning mechanism is determined. Screen printing of polyvinyl alcohol is currently not possible as a low volatility solvent has not been discovered capable of polyvinyl alcohol dissolution. Stencil printing is possible although resolution attainable is outside the microfluidic regime. Consequently, 
current solvents, including but not limited to, water, ethylene glycol, dimethyl sulfoxide, cannot function as a printing medium as solvent evaporates in-screen clogging the screen. Two possible solutions include developing a method to ink jet print polyvinyl alcohol from water at low weight percentages or investigating less hydrolyzed versions of this material.

A wealth of materials are studied for the production of STCM platforms. The best material system to date includes the cellulose acetate-PVC system. Although this material system is rather consistent, it is not without limitations. Consequently, it may be worth considering alternative materials for device production.

\subsubsection{Potential chemical modification of materials}

As mentioned previously in this chapter, the optimal device fabrication strategy does have associated problems with adhesion and resolution. As a result, it is worth considering novel approaches to chemically modifying substrates circumventing the necessity for screen printing altogether. Three potential approaches discussed in detail in chapter 4 include: (1) microplasma surface modification (2) UV-mediated photoacid generator hydrolysis on acrylic materials and (3) UV-initiated photodecomposition of aliphatic ketones followed by the subsequent self-initiated grafting of acrylic acid on polyethylene.

Microplasma writing is interesting as it is amenable to roll-to-roll processing and results in a substantial contact angle change. However, the contact angle decrease is not permanent and over time begins to increase back to the original non-wettable state. This problem could be potentially mitigated by finding suitable storage protocols.

The UV-mediate techniques are both interesting and have preliminary data suggesting these techniques may be worth investigating further. The hydrolysis of acrylic materials with 
a photoacid generator could potentially be cost ineffective since inhibiting the dissolution of the modified material may require expensive co-polymer systems and the cost of the photoacid generator is substantial as well. The UV-initiated photografting technique results in great decrease in contact angle but is far from being optimized. Furthermore, the use of polyethylene, with a contact angle greater than the $90^{\circ}$ critical angle, inhibits confinement loss. The photografting of polyethylene could potentially be an attractive method if correctly optimized.

\subsection{Imperative for integration of versatile analyses}

A great deal of this project involves the optimization of a limited rigor and economically viable fabrication process for autonomous microfluidic platforms. Such criteria have been fulfilled via the thick film printing methodology. Nonetheless, for this technology to be taken seriously, it is imperative that a powerful and versatile application be integrated into the chip. Two possible techniques include the enzyme-linked immunosorbent assay and fluorescent resonance energy transfer.

\subsubsection{Enzyme-linked immunosorbent assay design}

A handful of simple reaction processes reveal the capacity of the surface tensionconfined microfluidic technology to facilitate chemistry. However, analyses of clinical interest have yet to be demonstrated within the inherently uncomplicated surface tension-

confined microfluidic platform. Consequently, as a model reaction, it is proposed to investigate the enzyme-linked immunosorbent assay (ELISA). 
The ELISA technique was developed at the Stockholm University by Perlmann and Engvall (Engvall, 1977, Engvall \& Perlman, 1971). The ELISA technique was first demonstrated by quantitatively measuring the concentration of immunoglobulin $\mathrm{G}$ (IgG) in rabbit serum utilizing alkaline phosphatase as the reporting label through hydrolysis of a known concentration of substrate material. ELISA development was an effort to eliminate the method of utilizing radioactive reporter labels which required expensive analytical equipment to yield readings while the radioactivity presented significant safety concerns (Lequin, 2005).

ELISA has since garnered a considerable amount of interest for clinical diagnostics. Subsequent research regarding the ELISA technique resulted in methods for the detection of parasites (Ljungstrom et al., 1974, Voller et al., 1975) and other microbes (Engvall, 1977) as well as applications in oncology and endocrinology (Seppala et al., 1978, Sipponen et al., 1976, Uotila et al., 1981), and DNA hybridization (Palecek et al., 2002). Furthermore, the efficacy of enzyme-based immunoassays have been demonstrated in the detection of hepatitis $\mathrm{B}$ antigens and antibodies indicating the presence of rubella, toxoplasma and the human immunodeficiency virus (Lequin, 2005).

Although the ELISA technique was successfully automated in the early 1980s, the format of the analysis required reagent-expensive 96-well titer plates (Lequin, 2005). This is particularly troublesome when reagent is either very expensive, exists in small quantities or requires invasive procedures to attain. Furthermore, enzymatic hydrolysis must be conducted for detection resulting in an additional time-intensive incubation step. To mitigate the time requirement, fluorescent labels have been developed (Voller, 1978). The proposed 
investigation seeks to develop a fluorometric format ELISA technique integrated into an onchip platform in order to yield real-time results.

The idea of developing a microfluidic ELISA platform is not entirely revolutionary. Microfluidic ELISA methods have been developed for the detection of rat IgG from a hybridoma cell culture via a centrifugal conjugation mechanism in compact disc platforms (Lai et al., 2004). A heterogeneous sandwich ELISA has been developed in a PDMS platform for the detection of a model analyte (sheep $\operatorname{IgM}$ ) with a threshold sensitivity of nanomolar concentration utilizing a cooled charge coupled device (CCD) camera (Eteshola \& Balberg, 2004, Eteshola \& Leckband, 2001). Microfluidic platforms have been developed that can conduct several ELISA analyses in parallel for the detection of anti-streptavidin with picomolar sensitivity (Hermann et al., 2006). An electrochemical sandwich ELISA has been developed for the detection of redox active enzyme substrates with nanomolar sensitivity (Rossier \& Girault, 2001). A disposable microfluidic platform has been developed for the detection of botulinum toxin via a colorimetric ELISA with the tectonic integration of channel networks, valves and filters (Eddington et al., 2004). A micro-based ELISA system fabricated for the detection of interferon- $\gamma$ utilizing a dam structure of polystyrene beads for conjugation with a thermal lens microscope for detection (Sato et al., 2004). Consequently, the detection mechanism is greatly simplified and detection time is drastically reduced.

The chosen biomolecule conjugation system has already been selected based on studies conducted by previous groups (Sato et al., 2004). Mouse anti-recombinant human interferon- $\gamma$ (R \& D Systems, Inc.) will act as the capture antibody responsible for detecting the presence of the selected antigen of recombinant human interferon- $\gamma$ (R \& D Systems, Inc.) in a serum sample. After passing the serum with unknown concentration of antigen 
through the microfluidic device, a solution containing biotinylated anti-recombinant interferon- $\gamma$ antibody from goat (R \& D Systems, Inc.) will be introduced to the system. Lastly, Texas Red labeled streptavidin S-872 (Invitrogen) will pass through the device to complex with the biotinylated antibody for detection. Lastly, a copious amount of phosphate buffer solution will be injected into the device to wash all excess labeled streptavidin through the device as not to interfere with fluorescence imaging of the antigen-antibody complexes. Fluorescence will be measured with a Nikon TS-100 inverted microscope and the associated Texas Red filter cube. The advantage to the fluorescent approach is that detection is immediate after the last conjugation step since a fluor-labeled streptavidin is utilized as opposed to the enzyme-labeled analog. Specifically, this eliminates the requirement for the introduction of a substrate solution for hydrolysis which requires lengthy incubation steps for the hydrolysis kinetics to reach steady state. This advance reduces the steps required in the implementation protocol as well as drastically reducing the time requirement for important results that may indicate a patient start therapy.

An initial design for the multi-tiered platform is demonstrated in figure 5.1. Notice that all fluids are introduced into a common port. Each successive laminar slug of fluid pushes the preceding slug through the device into the blotter material. Consequently, complexation occurs as the biomolecules in the sample are passed across the antibody coated cover slip. The phosphate buffer then pushes any residual biomolecules into the waste blotted material to eliminate interference. 


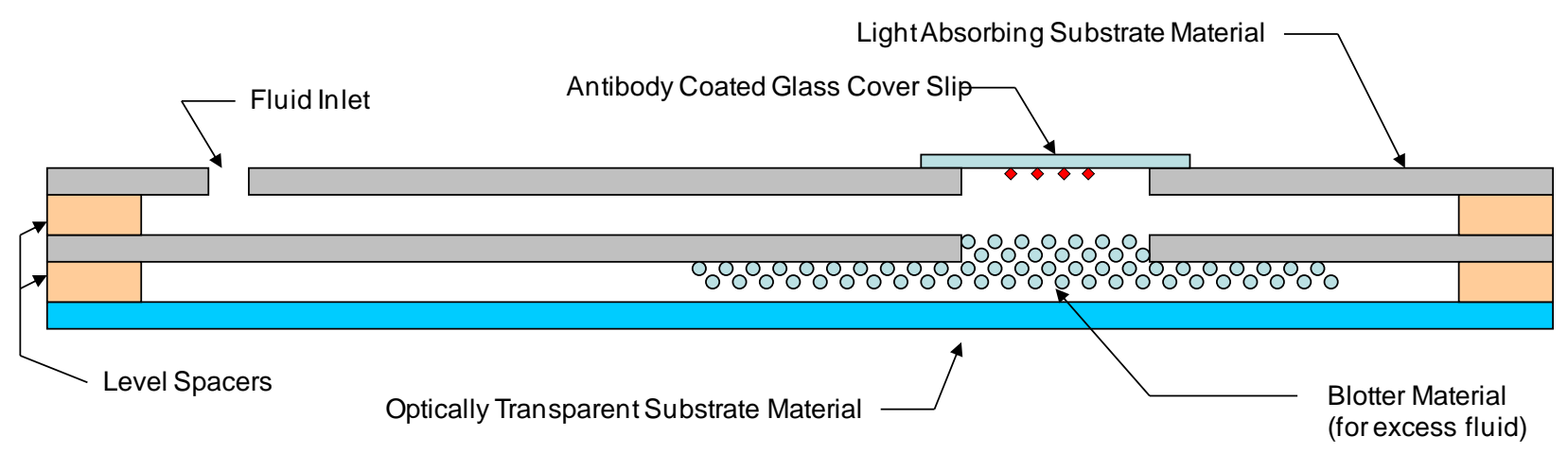

Figure 5.1 Side view of preliminary design of multi-tiered surface tension-confined microfluidic platform facilitating the ELISA technique in an on-chip format.

To inhibit interference from non-specific adsorbance of proteins onto the glass coverslip, the antibody coated cover-slip must be blocked with casein (Sato et al., 2004). A brief non-optimized protocol is as follows. A solution of capture antibody is prepared in phosphate buffered saline (PBS) at a concentration of 50 micrograms/milliliter. The glass substrate material submerged in this solution under weak agitation for 1 hour. Following the agitation, this solution and substrate are incubated overnight at $4{ }^{\circ} \mathrm{C}$. Following incubation, the substrate is washed with PBS. The spaces left on the substrate material that are not coated with antibody material are then blocked with casein (Merck, Biochem Grade) through submergence in $0.2 \%$ solution of casein for 1 hour. Lastly, the substrate is washed PBS one last time and stored in a refrigerator until use.

Lastly, the successful accomplishment of this task demonstrates the value associated with microfluidic system in a clinical sense. Although this will not be the first time that the ELISA technique has been performed in a microfluidic system, this will most certainly be one of the cheapest and easiest systems facilitating this analysis to date. Consequently, the surface-directed ELISA system is very attractive for commercial deployment. 


\subsubsection{Fluorescent resonance energy transfer on-chip}

One means for biological sensor development involves hybridizing labeled nucleic acids with a target DNA sample. This can be achieved via fluorescent resonance energy transfer (FRET - also known as Förster resonance energy transfer after the inventor of the technique).

FRET is the physical process by which energy is transferred non-radiatively from a donor chromophore to the acceptor chromophore via long range intermolecular dipole-dipole coupling (Clegg, 1995). This process can occur on a length scale of 1-10 nm. However, as the distance between the donor and acceptor fluors increases, the signal begins to drastically diminish.

This technique has been exploited for the development of DNA sensors detecting the presence of azoospermia (Jung et al., 2007, Kim et al., 2007). This technique is very attractive as the hybridization is a one-step process rather than tedious multistep cycling techniques like polymerase chain reaction (Cady et al., 2004, Khandurina et al., 2000, Zhang et al., 2006). Furthermore, as this is a DNA-based assay, it is more stable than $\mathrm{pH}$ and temperature sensitive enzyme-based assays and it is more direct than protein assays since the production of a target protein is most often the manifestation of a mutation in DNA or presence of infectious organism.

The assay involves introducing a ringed DNA sample (molecular beacon) that is complimentary to a target DNA sequence. On each end of the molecular beacon are attached chromophores. In one example, the chromophores are a donor and quencher pair (FAMDabcyl, respectively) resulting in signal gain through the introduction of the target DNA (Kim et al., 2007). In contrast, the chromophores can also be a donor and a acceptor (TET 
and TAMRA, respectively) resulting in loss of signal through introduction of target DNA (Jung et al., 2007). The intermediate sequence of oligonucleotides can be custom made to hybridize with the known target DNA sequence (BioBasic, Inc.; Markham, Ontario). Since the assay requires bringing a fluid sample containing the target DNA in close proximity to a fluid sample with the molecular beacon, efficient microfluidic mixing is mandated. The assay is illustrated in figure 5.2 (adapted from literature sources).

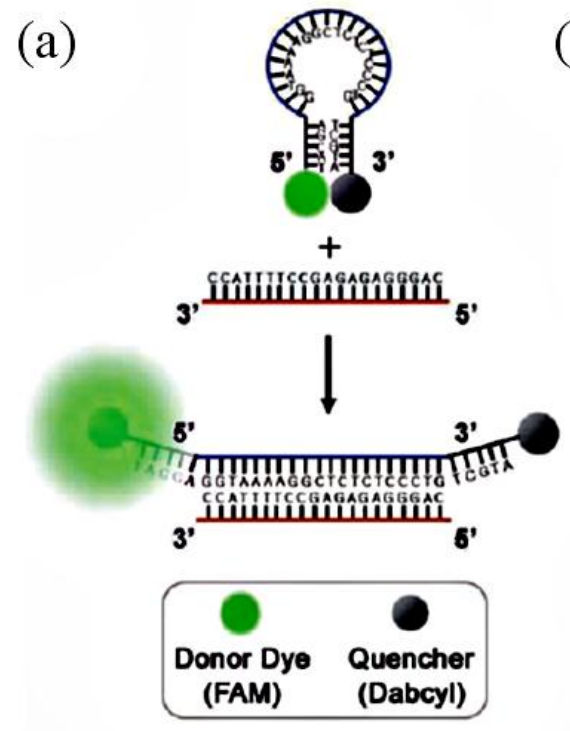

(b)

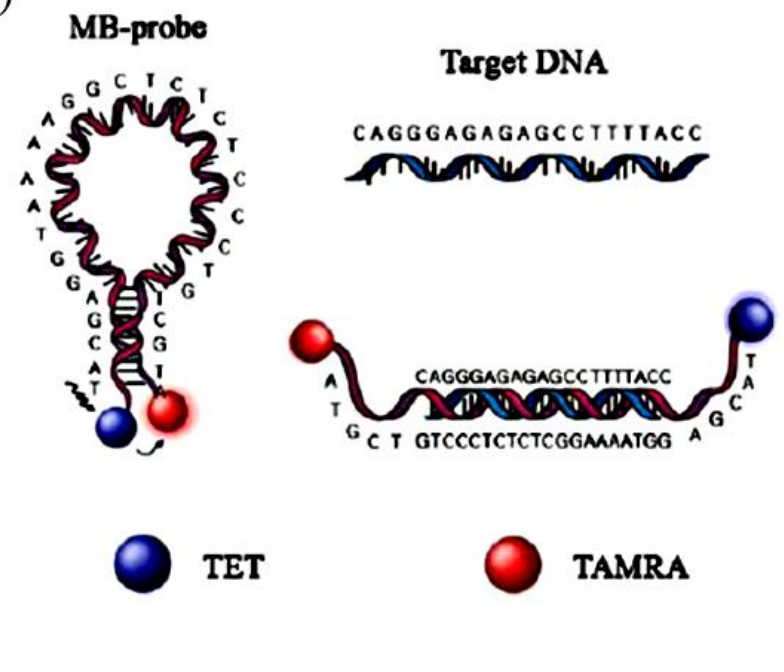

Figure 5.2 Demonstration of molecular beacon systems. (a) Hybridization results in fluorescent signal gain (Kim et al., 2007). (b) Hybridization results in signal loss (Jung et al., 2007).

Since it is imperative to mix the fluid samples in this assay, this investigation affords the opportunity to demonstrate the capacity of the STCM platforms to perform passive mixing through chaotic advection (see section 3.3.3) as well as exhibit the facility of the chip to perform a powerful and versatile diagnostic technique. Since the mixing approach generates velocity gradients through curvature manipulation, mixing can only occur near the advancing interface. Consequently, it is proposed that the assay is conducted as follows. Initially a very small sample of molecular beacon serum should be introduced to the device 
( $\sim 5$ microliters). This will be followed by the introduction of a small sample of serum which may contain the target DNA. The two fluid ducts will then be pushed through the device by introducing a larger amount of buffer solution or water. If energy discontinuities are patterned in the path of the advancing fluid, the two small plugs of molecular beacon serum and analyzed serum will mix as the fluid advances.

The suggested designs for mixing pattern are demonstrated in figure 5.3. One pattern includes the control pattern devoid of surface energy discontinuities. Another pattern is the checkerboard mixer utilized in section 3.3.3. An alternative pattern includes the herringbone pattern developed by Stroock, et al., utilized in other chaotic advection micromixing studies (Stroock et al., 2002, Stroock \& Whitesides, 2003). The last pattern is an improvement on the herringbone pattern using the Weierstrass fractal function for a more random herringbone pattern utilizing a fractal dimension of $D=1.25$ as determined to be the optimal configuration through computational fluid dynamics studies by Camesasca (Camesasca et al., 2006). The strategy of exploiting the Weierstrass fractal function (equation 5.1), as opposed to the ordered herringbone pattern for geometric design is the concept that one cannot achieve true chaotic advection unless a truly chaotic design criteria is utilized.

$$
W(x)=\sum_{n=1}^{\infty} \frac{\cos \left(2^{n} x\right)}{2^{n(2-D)}}
$$




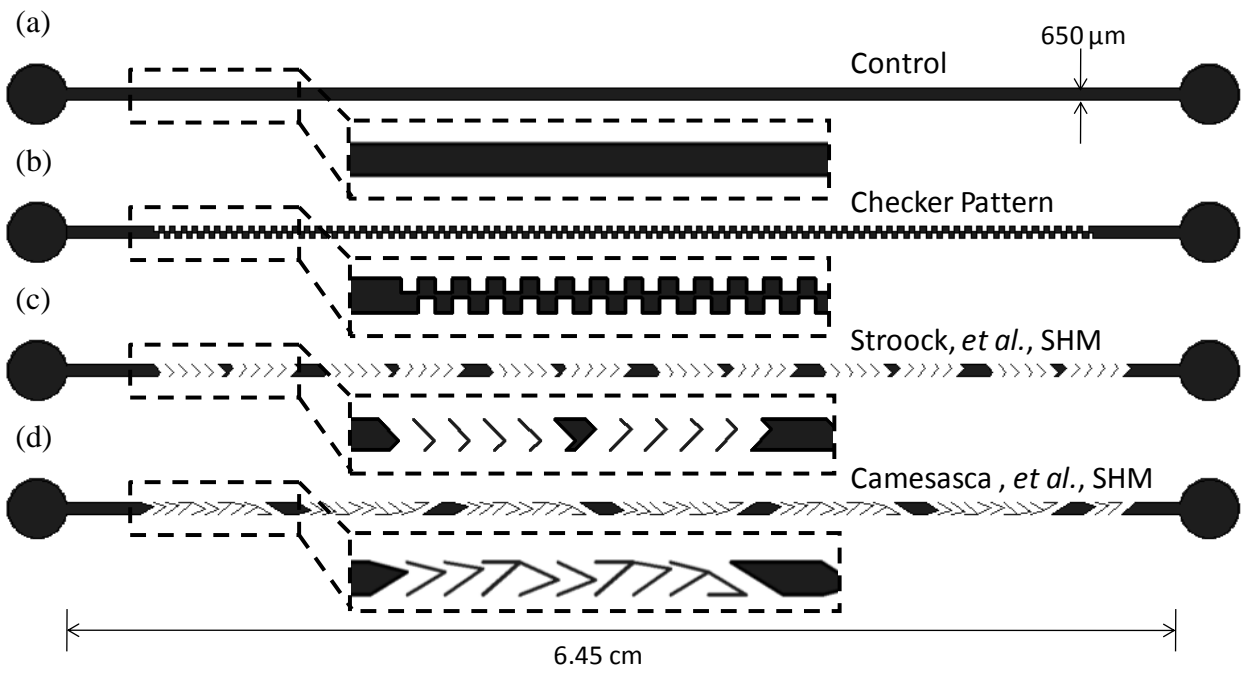

Figure 5.3 Mixer design patterns for $6 \mathrm{~cm}$ long paths. (a) Control pattern, (b) checker board (see section 3.3.3) (c) staggered herringbone adapted from Stroock, et al., and (a) Weierstrass herringbone pattern adapted from Camesasca, et al.

It is suggested that this study be commenced immediately. Preliminary designs exist following the work of Stroock, et al., and Camesasca, et al., are demonstrated by figure 5.3 for a $6.45 \mathrm{~cm}$ long channel with a path width of $650 \mu \mathrm{m}$ and a gap height of $229 \mu \mathrm{m}$. Not only will success demonstrate the utility of the surface tension-confined microfluidic platform for biosensing applications, it also gives an opportunity to quantify the mixing efficiency demonstrated in section 3.3.3 through fluorescent microscopy. Effectively completing this investigation should exhibit the capacity of the STCM device to conduct 'lab on a chip'-type processes as well as establish a framework for designing passive mixers via surface energy disruption for future studies.

\subsection{Current Disadvantages of STCM Platforms}

Although this manuscript largely triumphs the surface tension-confined microfluidic (STCM) platform, the platforms fabricated as discussed herein do have drawbacks. Specifically, there are disadvantages associated with platform fabrication and 
implementation. This section seeks to explicitly discuss such problems enabling future researchers of this approach to foresee these problems without necessarily having to discover them on their own. Subsequently, we hope this section will lower the learning curve for new researchers of this approach.

First and foremost, there are several problems associated with platform fabrication. In comparison with other photolithographic techniques capable of nanometer resolution, the thick film printing process is only capable of $\sim 300$ micron resolution using in-house screen fabrication techniques. Better resolution may be attainable by purchasing custom made screens commercially (e.g. Sefar Holding Inc.) although the minimum screen opening dimension is roughly 28 microns establishing the minimum realizable resolution in a best case scenario. A related disadvantage pertaining to path resolution is as path width decreases, alignment becomes more troublesome when performed visually in the laboratory. Perhaps future researchers can automate the alignment procedure with a photo-mask aligner mitigating inconsistencies associated with poor alignment. In addition, the screen printed paths do not seem to achieve adequate adhesion in the case of the PVC-cellulose acetate system. Better adhesion is achieved for the polystyrene-cellulose acetate system as chain entanglement is facilitated by the tetraglyme solvent but the processing of the polystyrene substrate to correct size is rather tedious. Lastly, due to the inherent low volatility of the tetraglyme (i.e. tetraethylene glycol dimethyl ether) solvent necessary to inhibit screen clogging, it is speculated that residual solvent is present in the hydrophilic paths for a long amount of time post-patterning. For this last reason, it may be of interest to develop an ink jet printing process to eliminate the necessity of a solvent that may remain post-patterning interacting negatively with biomolecules or analytes of interest. 
With regard to implementation, the STCM platform is completely autonomous relying upon capillary forces for fluidic manipulation. Although this is advantageous when the user is not experienced with chemical analyses, this can also be a drawback as it eliminates the ability to orchestrate complex reaction processes as valving and flowrate control is not feasible. Possible solutions may include the integration of less complicated detection methods or assays requiring minimal steps. Furthermore, current fluid volumes required for implementation are on the order of 10 microliters. Although this is a minimal quantity of fluid, this volume of fluid is still large enough to be manipulated with micropipetting systems in a well-plate format. The total quantity of fluid could potentially be further minimized by finding plate spacing materials less than the $116 \mu \mathrm{m}$ thick material utilized in the development of the 2D STCM platforms.

Lastly, the author of this text ultimately envisions the surface tension-confined microfluidic platform to be manufactured via roll-to-roll processing of polymeric materials. For this to become a realizable scenario, the previously discussed disadvantages need to be addressed accordingly.

\subsection{Conclusions}

The surface tension-confined microfluidic platform is very appealing with regards to the limited cost and rigor associated with device fabrication. Not only are the autonomous devices amenable to mass production, the lack of sophisticated excess componentry required for implementation makes the platforms inherently fail-safe. Although a consistent fabrication protocol exists for the production such devices, there is opportunity for improvement. 
Since delamination and ultimate resolution are a problem for the thick film-printed devices, alternative patterning techniques exist enabling production through stencil printing and potentially ink jet printing. Furthermore, possibilities exist for path patterning via chemical means. For example, photoacidic hydrolysis and UV-grafting of acrylic acid may facilitate chemical methods to make virtual paths.

In addition to improvements in manufacturing technology, it is imperative to demonstrate that the surface tension-confined microfluidic platforms can perform powerful and versatile chemical reaction processes. Two model assays have been elucidated and preliminary designs exist for the integration of the assays into an on-chip format. The enzyme-linked immunosorbent assay is attractive due to how ubiquitous this technique is utilized clinically. The molecular beacon fluorescent resonance energy transfer technique is appealing since it requires minimal steps and can be easily tailored for a wide variety of target DNA samples. Furthermore, the FRET technique also enables a fundamental study on micromixing efficiency within the STCM platform.

In conclusion, the STCM technology described within this text has a number of advantages. Some advantages include, but are not limited to: (1) device cost is minimized (2) fabrication is simplified (3) platforms are completely autonomous and therefore not susceptible to malfunction (5) fabrication is versatile and can be tailored for specific applications (6) the production of the STCM platform can be implemented continuously rather than in a batch format and (7) simple reaction processes have been implemented within STCM platforms indicating the platform is capable of conducting 'lab on a chip' reaction processes. Before this technology gains wide-spread acceptance, it is attractive to implement more powerful diagnostic methods within the platform. Consequently, it is suggested that 
immediate investigation follow the path of surface tension-confined microfluidic sensor development.

\subsection{References}

Cady, N. C., Stelick, S., Kunnavakkam, M. V. \& Batt, C. A. (2004). Sensors and Actuators B $107,332-341$.

Camesasca, M., Kaufman, M. \& Manas-Zloczower, I. (2006). Journal of Micromechanics and Microengineering 16, 2298-2311.

Clegg, R. M. (1995). Current Opinion in Biotechnology 6, 103-110.

Eddington, D. T., Tepp, W. H., Johnson, E. A. \& Beebe, D. J. (2004). Electrophoresis 25, 1705-1713.

Engvall, E. (1977). Med Biol 55, 193-200.

Engvall, E. \& Perlman, P. (1971). Immunochemistry 8, 871-874.

Eteshola, E. \& Balberg, M. (2004). Biomedical Microdevices 6, 7-9.

Eteshola, E. \& Leckband, D. (2001). Sensors and Actuators B 72, 129-133.

Herrmann, M., Veres, T. \& Tabrizian, M. (2006). Lab on a Chip 6, 555-560.

Jung, J., Chen, L., Lee, S., Kim, S., Seong, G. H., Choo, J., Lee, E. K. \& Oh, C. H. (2007). Analytical and Bioanalytical Chemistry 387, 2609-2615.

Khandurina, J., McKnight, T. E., Jacobson, S. C., Waters, L. C., Foote, R. S. \& Ramsey, J. M. (2000). Anal. Chem 72, 2995-3000.

Kim, S., Chen, L., Lee, S., Seong, G. H., Choo, J., Lee, E. K. \& Oh, C. H. (2007). Analytical Sciences 23, 401-405.

Lai, S., Wang, S., Luo, J., Lee, L., Yang, S. T. \& Madou, M. (2004). Anal. Chem 76, $1832-$ 1837.

Lequin, R. M. (2005). Clinical Chemistry 51, 2415-2418.

Ljungstrom, I., Engvall, E. \& Ruitenberg, E. J. (1974). Parasitology 69, 2. 
Palecek, E., Kizek, R., Havran, L., Billova, S. \& Fojta, M. (2002). Analytica Chimica Acta 469, 73-83.

Rossier, J. S. \& Girault, H. H. (2001). Lab on a Chip 1, 153.

Sato, K., Yamanaka, M., Hagino, T., Tokeshi, M., Kimura, H. \& Kitamori, T. (2004). Lab on a Chip 4, 570-575.

Seppala, M., Rutanen, E. M., Heikinheimo, M., Jalanko, H. \& Engvall, E. (1978). Int J Cancer 21, 265-267.

Sipponen, P., Ruoslahti, E., Vuento, M., Engvall, E. \& Stenman, U. (1976). Acta Hepatogastroenterol 23, 276-279.

Stroock, A. D., Dertinger, S. K. W., Ajdari, A., Mezic, I., Stone, H. A. \& Whitesides, G. M. (2002). Science 295, 647-651.

Stroock, A. D. \& Whitesides, G. M. (2003). Acc. Chem. Res 36, 597-604.

Uotila, M., Ruoslahti, E. \& Engvall, E. (1981). J Immunol Methods 42, 11-15.

Voller, A. (1978). Journal of Clinical Pathology 31, 507-520.

Voller, A., Huldt, G., Thors, C. \& Engvall, E. (1975). Br Med J 1, 659-661.

Watanabe, M. (2007). Sensors and actuators. B, Chemical 122, 141-147.

Zhang, C., Xu, J., Ma, W. \& Zheng, W. (2006). Biotechnol. Adv 24, 243-284. 


\section{Bibliography}

1. $\quad \mathrm{AB}, \mathrm{C}$. , Inkjet Model. In COMSOL Multiphysics, 2006; pp 1-16.

2. Abraham, D. B., Capillary Waves and Surface Tension: An Exactly Solvable Model. Physical Review Letters 1981, 47, (8), 545-548.

3. Abraham, D. B.; Reed, P., Diagonal interface in the two-dimensional Ising ferromagnet. J. Phys. A 1977, 10, (6).

4. Adamson, A. W.; Gast, A. P., Physical Chemistry of Surfaces, 6-thed. WileyInterscience: New York: 1997.

5. $\quad$ Albert, R. K.; Lakshminarayan, S.; Hildebrandt, J.; Kirk, W.; Butler, J., Increased surface tension favors pulmonary edema formation in anesthetized dogs' lungs. J Clin Invest 1979, 63, (5), 1015-1018.

6. Allmer, K.; Hult, A.; Ranby, B., Surface modification of polymers. I. Vapour phase photografting with acrylic acid. Journal of Polymer Science Part A Polymer Chemistry 1988, 26, (8), 2099-2111.

7. Bachofen, H.; Hildebrandt, J.; Bachofen, M., Pressure-volume curves of air-and liquid-filled excised lungs-surface tension in situ. Journal of Applied Physiology 1970, 29, (4), 422-431.

8. Bachofen, H.; Schurch, S.; Urbinelli, M.; Weibel, E. R., Relations among alveolar surface tension, surface area, volume, and recoil pressure. Journal of Applied Physiology 1987, 62, (5), 1878-1887.

9. Bartell, F. E.; Bartell, L. S., Quantitative Correlation of Interfacial Free Surface Energies*. Journal of the American Chemical Society 1934, 56, (11), 2205-2210.

10. Beake, B. D.; Ling, J. S. G.; Leggett, G. J., Correlation of friction, adhesion, wettability and surface chemistry after argon plasma treatment of poly (ethylene terephthalate). J. Mater. Chem 1998, 8, 2845-2854.

11. Beebe, D. J.; Moore, J. S.; Yu, Q.; Liu, R. H.; Kraft, M. L.; Jo, B. H.; Devadoss, C., Microfluidic tectonics: A comprehensive construction platform for microfluidic systems. Proceedings of the National Academy of Sciences 2000, 97, (25), 13488-13493.

12. Bird, R. B.; Stewart, W. E.; Lightfoot, E. N., Transport Phenomena. 2nd ed.; John Wiley \& Sons, Inc. : New York, 2002; p 112. 
13. Bouaidat, S.; Hansen, O.; Bruus, H.; Berendsen, C.; Bau-Madsen, N. K.; Thomsen, P.; Wolff, A.; Jonsmann, J., Surface-directed capillary system; theory, experiments and applications. Lab on a Chip 2005, 5, (8), 827-836.

14. Boys, C. V., Soap Bubbles: Their Colours and the Forces which Mold Them: Being the Substance of Many Lectures Delivered to Juvenile and Popular Audiences: with the Addition of Several New and Original Sections. Dover Publications: 1959.

15. Brakke, K. A., The Surface Evolver. Experimental Mathematics 1992, 1, (2), 141165.

16. Bristol, J. Emulsion polymerization of vinyl acetate. 2,614,088, 1952.

17. Brooks, T.; Keevil, C. W., A simple artificial urine for the growth of urinary pathogens. Letters in Applied Microbiology 1997, 24, (3), 203-206.

18. Buff, F. P.; Lovett, R. A.; Stillinger Jr, F. H., Interfacial Density Profile for Fluids in the Critical Region. Physical Review Letters 1965, 15, (15), 621-623.

19. Burns, M. A., An Integrated Nanoliter DNA Analysis Device. Science 1998, 282, (5388), 484-487.

20. Butler, J. E.; Lue, E. P.; Navarro, P.; Christiansen, B., Comparative studies on the interaction of proteins with a polydimethylsiloxane elastomer. I. Monolayer protein capture capacity(PCC) as a function of protein $\mathrm{pl}$, buffer $\mathrm{pH}$ and buffer ionic strength. Journal of Molecular Recognition 1997, 10, (1), 36-51.

21. Butler, J. E.; Navarro, P.; Lue, P., Comparative studies on the interaction of proteins with a polydimethylsiloxane elastomer. II. The comparative antigenicity of primary and secondarily adsorbed IgG 1 and IgG 2 a and their non-adsorbed counterparts. Journal of Molecular Recognition 1997, 10, (1), 52-62.

22. Cady, N. C.; Stelick, S.; Kunnavakkam, M. V.; Batt, C. A., Real-time PCR detection of Listeria monocytogenes using an integrated microfluidics platform. Sensors and Actuators B 2004, 107, (1), 332-341.

23. Camesasca, M.; Kaufman, M.; Manas-Zloczower, I., Quantifying Fluid Mixing with the Shannon Entropy. Macromolecular theory and simulations 2006, 15, (8), 595-607.

24. Camesasca, M.; Kaufman, M.; Manas-Zloczower, I., Staggered passive micromixers with fractal surface patterning. Journal of Micromechanics and Microengineering 2006, 16, (11), 2298-2311.

25. Camesasca, M.; Manas-Zloczower, I.; Kaufman, M., Entropic characterization of mixing in microchannels. Journal of Micromechanics and Microengineering 2005, 15, (11), 2038-2044. 
26. Chan, C. M.; Ko, T. M.; Hiraoka, H., Polymer surface modification by plasmas and photons. Surface science reports 1996, 24, (1-2), 1-54.

27. Chen, C. F.; Kung, C. F.; Chen, H. C.; Chu, C. C.; Chang, C. C.; Tseng, F. G., A microfluidic nanoliter mixer with optimized grooved structures driven by capillary pumping. Journal of Micromechanics and Microengineering 2006, 16, (7), 1358-1365.

28. Chiang, W. H.; Cochey, M.; Virnelson, R. C.; Sankaran, R. M., Nonlithographic fabrication of surface-enhanced Raman scattering substrates using a rastered atmosphericpressure microplasma source. Applied Physics Letters 2007, 91, 021501.

29. Cho, B. O.; Hwang, S. W.; Lee, G. R.; Moon, S. H., Angular dependence of the redeposition rates during $\mathrm{SiO}$ etching in a $\mathrm{CF}$ plasma. Journal of Vacuum Science \& Technology A: Vacuum, Surfaces, and Films 2001, 19, 730.

30. Choban, E. R.; Markoski, L. J.; Wieckowski, A.; Kenis, P. J., Microfluidic fuel cell based on laminar flow. Journal of Power Sources 2004, 128, (1), 54-60.

31. Choi, C. H.; Westin, K. J. A.; Breuer, K. S., To slip or not to slip water flows in hydrophilic and hydrophobic microchannels. Proceedings of IMECE 2002, 33707, 13-16.

32. Clegg, R. M., Fluorescence resonance energy transfer. Current Opinion in Biotechnology 1995, 6, (1), 103-110.

33. Concus, P.; Finn, R., On the Behavior of a Capillary Surface in a Wedge. Proceedings of the National Academy of Sciences 1969, 63, (2), 292-299.

34. Dann, J. R., Forces involved in the adhesive process. I. Critical surface tensions of polymeric solids as determined with polar liquids. J. Colloid Interface Sci 1970, 32, (2), 302331.

35. Darhuber, A. A., Generation of high-resolution surface temperature distributions. Journal of Applied Physics 2002, 91, (9), 5686.

36. Darhuber, A. A.; Davis, J. M.; Troian, S. M.; Reisner, W. W., Thermocapillary actuation of liquid flow on chemically patterned surfaces. Physics of Fluids 2003, 15, (5), 1295-1304.

37. Darhuber, A. A.; Troian, S. M., Principles of microfluidic actuation by modulation of surface stresses. Annual Review of Fluid Mechanics 2005, 37, (1), 425-455.

38. Darhuber, A. A.; Troian, S. M.; Miller, S. M.; Wagner, S., Morphology of liquid microstructures on chemically patterned surfaces. Journal of Applied Physics 2006, 87, (11), 7768-7775. 
39. Darhuber, A. A.; Troian, S. M.; Reisner, W. W., Dynamics of capillary spreading along hydrophilic microstripes. Physical Review E 2001, 64, (3), 31603.

40. Darhuber, A. A.; Valentino, J. P.; Davis, J. M.; Troian, S. M.; Wagner, S., Microfluidic actuation by modulation of surface stresses. Applied Physics Letters 2003, 82, (4), 657-659.

41. Defay, R.; Prigogine, I., Surface Tension and Adsorption, translated by DH Everett. 1st ed.; Wiley-Interscience: New York, 1966; Vol. 319.

42. Delamarche, E.; Bernard, A.; Schmid, H.; Bietsch, A.; Michel, B.; Biebuyck, H., Microfluidic Networks for Chemical Patterning of Substrates: Design and Application to Bioassays. J. Am. Chem. Soc 1998, 120, (3), 500-508.

43. Dietrich, S.; Popescu, M. N.; Rauscher, M., Wetting on structured substrates. Journal of Physics: Condensed Matter 2005, 17, (9), S577-S593.

44. Dupré, A. M.; Dupré, P., Théorie mécanique de la chaleur. Gauthier-Villars: 1869.

45. Eddington, D. T.; Tepp, W. H.; Johnson, E. A.; Beebe, D. J., Microfluidic tectonics platform: A colorimetric, disposable botulinum toxin enzyme-linked immunosorbent assay system. Electrophoresis 2004, 25, 1705-1713.

46. Ellerby, L. M.; Nishida, C. R.; Nishida, F.; Yamanaka, S. A.; Dunn, B.; Valentine, J. S.; Zink, J. I., Encapsulation of proteins in transparent porous silicate glasses prepared by the sol-gel method. Science 1992, 255, (5048), 1113.

47. Engvall, E., Quantitative enzyme immunoassay (ELISA) in microbiology. Med Biol 1977, 55, (4), 193-200.

48. Engvall, E.; Perlman, P., Enzyme-linked immunosorbent assay (ELISA). Quantitative assay of immunoglobulin G. Immunochemistry 1971, 8, (9), 871-874.

49. Erickson, D.; Li, D., Integrated microfluidic devices. Analytica Chimica Acta 2004, 507, (1), 11-26.

50. Eteshola, E.; Balberg, M., Microfluidic ELISA: On-Chip Fluorescence Imaging. Biomedical Microdevices 2004, 6, (1), 7-9.

51. Eteshola, E.; Leckband, D., Development and characterization of an ELISA assay in PDMS microfluidic channels. Sensors and Actuators B 2001, 72, (2), 129-133.

52. Fisher, M. P. A.; Fisher, D. S.; Weeks, J. D., Agreement of Capillary-Wave Theory with Exact Results for the Interface Profile of the Two-Dimensional Ising Model. Physical Review Letters 1982, 48, (5), 368. 
53. Fowkes, F. M., Additivity of intermolecular forces at interfaces. I. Determination of the contribution to surface and interfacial tensions of dispersion forces in various liquids. The Journal of Physical Chemistry 1963, 67, (12), 2538-2541.

54. Garciá, A. A.; Cherian, S.; Park, J.; Gust, D.; Jahnke, F.; Rosario, R., PhotonControlled Phase Partitioning of Spiropyrans. Letters.

55. Gau, H.; Herminghaus, S.; Lenz, P.; Lipowsky, R., Liquid Morphologies on Structured Surfaces: From Microchannels to Microchips. Phys. Rev. Lett 1998, 81, 1253.

56. Gibbs, J. W., The Collected Works of J. Willard Gibbs. Yale Univ. Press: New Haven, 1906; Vol. 1, p 236.

57. Gill, I.; Ballesteros, A., Bioencapsulation within synthetic polymers(Part 1): sol-gel encapsulated biologicals. Trends in Biotechnology 2000, 18, (7), 282-296.

58. Good, R. J.; Girifalco, L. A., A theory for estimation of surface and interfacial energies. III. Estimation of surface energies of solids from contact angle data. The Journal of Physical Chemistry 1960, 64, (5), 561-565.

59. Good, W. R., A comparison of contact angle interpretations. J. Colloid Interface Sci 1973, 44, 63.

60. Goodwin, S. B.; Schneider, R. E.; Fry, W. E., Use of cellulose-acetate electrophoresis for rapid identification of allozyme genotypes of Phytophthora infestans. Plant disease 1995, 79, (11), 1181-1185.

61. Grigoriev, R. O., Chaotic mixing in thermocapillary-driven microdroplets. Physics of Fluids 2005, 17, 033601.

62. Henriksson, U.; Eriksson, J. C., Thermodynamics of Capillary Rise: Why Is the Meniscus Curved? Journal of Chemical Education 2004, 81, (1), p1505.

63. Herring, C., The Use of Classical Macroscopic Concepts in Surface Energy Problems. In The Structure and Properties of Solid Surfaces, Gomer, R.; Smith, C. S., Eds. University of Chicago Press: Chicago, 1953; p 5.

64. Herrmann, M.; Veres, T.; Tabrizian, M., Enzymatically-generated fluorescent detection in micro-channels with internal magnetic mixing for the development of parallel microfluidic ELISA. Lab on a Chip 2006, 6, 555-560.

65. Holmes, E. P. J., Handbook of Thick Film Technology. 2 ed.; Emerald Group Publishing Limited: Bradford, UK, 1976.

66. Hong, J. W.; Quake, S. R., Integrated nanoliter systems. Nature Biotechnology 2003, 21, (10), 1179-1183. 
67. Huiliang Wang, H. R. B., Aliphatic Ketones as Photoinitiators for Photografting. Macromolecular Rapid Communications 2004, 25, (13), 1257-1262.

68. Iserles, A., A First Course in the Numerical Analysis of Differential Equations. Cambridge University Press: 1996.

69. Jayashree, R. S.; Gancs, L.; Choban, E. R.; Primak, A.; Natarajan, D.; Markoski, L. J.; Kenis, P. J. A., Air-Breathing Laminar Flow-Based Microfluidic Fuel Cell. J. Am. Chem. Soc 2005, 127, (48), 16758-16759.

70. Jiao, Z.; Nguyen, N. T.; Huang, X.; Ang, Y. Z., Reciprocating thermocapillary plug motion in an externally heated capillary. Microfluidics and Nanofluidics 2007, 3, (1), 39-46.

71. John, E.; Edward, P. C. Production of polyvinyl acetate beads and emulsions. CA $565,080,1958$.

72. John, J. H. Production of polyvinyl acetate. CA 815,109, 1969.

73. Johnson, T. J.; Ross, D.; Locascio, L. E., Rapid microfluidic mixing. Anal. Chem 2002, 74, (1), 45-51.

74. Juncker, D.; Schmid, H.; Drechsler, U.; Wolf, H.; Wolf, M.; Michel, B.; de Rooij, N.; Delamarche, E., Autonomous microfluidic capillary system. Anal. Chem 2002, 74, (24), 6139-6144.

75. Jung, J.; Chen, L.; Lee, S.; Kim, S.; Seong, G. H.; Choo, J.; Lee, E. K.; Oh, C. H., Fast and sensitive DNA analysis using changes in the FRET signals of molecular beacons in a PDMS microfluidic channel. Analytical and Bioanalytical Chemistry 2007, 387, (8), 26092615.

76. Kamholz, A. E.; Schilling, E. A.; Yager, P., Optical Measurement of Transverse Molecular Diffusion in a Microchannel. Biophysical Journal 2001, 80, (4), 1967-1972.

77. Kataoka, D. E., Patterning liquid flow on the microscopic scale. Nature 1999, 402, 794-797.

78. Khandurina, J.; McKnight, T. E.; Jacobson, S. C.; Waters, L. C.; Foote, R. S.; Ramsey, J. M., Integrated system for rapid PCR-based DNA analysis in microfluidic devices. Anal. Chem 2000, 72, (13), 2995-3000.

79. Khandurina, J.; McKnight, T. E.; Jacobson, S. C.; Waters, L. C.; Foote, R. S.; Ramsey, J. M., Integrated system for rapid PCR-based DNA analysis in microfluidic devices. Anal. Chem 2000, 72, (13), 2995-3000. 
80. Kim, S.; Chen, L.; Lee, S.; Seong, G. H.; Choo, J.; Lee, E. K.; Oh, C. H., Rapid DNA Hybridization Analysis Using a PDMS Microfluidic Sensor and a Molecular Beacon. Analytical Sciences 2007, 23, (4), 401-405.

81. Ko, J. S.; Yoon, H. C.; Yang, H.; Pyo, H. B.; Chung, K. H.; Kim, S. J.; Kim, Y. T., A polymer-based microfluidic device for immunosensing biochips. Lab on a Chip 2003, 3, 106113.

82. Koplik, J.; Banavar, J. R.; Willemsen, J. F., Molecular dynamics of fluid flow at solid surfaces. Physics of Fluids A: Fluid Dynamics 1989, 1, 781.

83. Kubota, H.; Koyama, M., Photografting of methacrylic acid on low-density polyethylene film in presence of polyfunctional monomers. Journal of applied polymer science 1997, 63, (12), 1635-1641.

84. Lagally, E. T.; Simpson, P. C.; Mathies, R. A., Monolithic integrated microfluidic DNA amplification and capillary electrophoresis analysis system. Sens. Actuators B 2000, $63,138-46$.

85. Lai, S.; Wang, S.; Luo, J.; Lee, L.; Yang, S. T.; Madou, M., Design of a compact disk-like microfluidic platform for enzyme-linked immunosorbent assay. Anal. Chem 2004, 76, (7), 1832-1837.

86. Lam, P.; Wynne, K. J.; Wnek, G. E., Surface-tension-confined microfluidics. Langmuir 2002, 18, (3), 948-951.

87. Lamanna, W. M.; Kessel, C. R.; Savu, P. M.; Cheburkov, Y.; Brinduse, S.; Kestner, T. A.; Lillquist, G. J.; Parent, M. J.; Moorhouse, K. S.; Zhang, Y., New ionic photo-acid generators (PAGs) incorporating novel perfluorinated anions. Proceedings -SPIE The International Society for Optical Engineering 2002, 817-828.

88. Lauga, E.; Brenner, M. P.; Stone, H. A., Microfluidics: The No-Slip Boundary Condition. In Handbook of Experimental Fluid Dynamics, 1 ed.; Springer: New York, 2005; Vol. 1.

89. Leamy, H. J.; Gilmer, G. H.; Jackson, K. A., Surface Physics of Materials. In Academic: New York, 1975; Vol. 1, p 121.

90. Lei, J.; Gao, J.; Zhou, R.; Zhang, B.; Wang, J., Photografting of acrylic acid on high density polyethylene powder in vapour phase. Polymer International 2000, 49, (11), $1492-$ 1495.

91. Lequin, R. M., Enzyme Immunoassay (EIA)/Enzyme-Linked Immunosorbent Assay (ELISA). Clinical Chemistry 2005, 51, (12), 2415-2418. 
92. Lewis, G. N.; Randall, M., Thermodynamics and the Free Energy of Chemical Substances. McGraw-Hill: 1923.

93. Lichtenberg, J.; de Rooij, N. F.; Verpoorte, E., Sample pretreatment on microfabricated devices. Talanta 2002, 56, (2), 233-266.

94. Liu, A.; He, F.; Wang, K.; Zhou, T.; Lu, Y.; Xia, X., Rapid method for design and fabrication of passive micromixers in microfluidic devices using a direct-printing process. Lab on a Chip 2005, 5, (9), 974-978.

95. Ljungstrom, I.; Engvall, E.; Ruitenberg, E. J., Proceedings: ELISA, enzyme linked immunosorbent assay-a new technique for sero-diagnosis of trichinosis. Parasitology 1974, $69,2$.

96. Ma, X.; Sun, Q.; Su, Y.; Wang, Y.; Jiang, Z., Antifouling property improvement of poly (vinyl butyral) ultrafiltration membranes through acid treatment. Separation and Purification Technology 2007, 54, (2), 220-226.

97. Madou, M., Lithography. In Fundamentals of Microfabrication, 1 ed.; CRC Press: Boca Raton, 1997; p 10.

98. Makal, U.; Wynne, K. J., Water induced hydrophobic surface. Langmuir 2005, 21, (9), 3742-3745.

99. Mann Jr, J. A., A free energy model for curved surfaces and curved thin films in emulsions. Journal of dispersion science and technology 2002, 23, (1-3), 199-207.

100. Mann Jr, J. A.; Romero, L.; Rye, R. R.; Yost, F. G., Flow of simple liquids down narrow V-grooves. Physical Review E 1995, 52, (4), 3967-3972.

101. Marengo-Rowe, A. J., Rapid electrophoresis and quantitation of haemoglobin on cellulose acetate. J Clin Pathol 1965, 18, (6), 790-2.

102. Martien, F. L., Vinyl alcohol polymers. In Encyclopedia of Polymer Science and Engineering

Mark, H. F.; Bikales, N. M.; Overberger, C. G.; Menges, G.; Kroschwitz, J. I., Eds. WileyInterscience: New York, 1987; Vol. 17.

103. Martinez, A. W.; Phillips, S. T.; Butte, M. J.; Whitesides, G. M., Patterned Paper as a Platform for Inexpensive, Low-Volume, Portable Bioassays*. Angew. Chem. Int. Ed. 2007, 46, 1318-1320.

104. Mastrangelo, C. H.; Burns, M. A.; Burke, D. T., Microfabricated devices for genetic diagnostics. Proceedings of the IEEE 1998, 86, (8), 1769-1787. 
105. Matsumoto, M.; Imai, K. Production of polyvinyl acetate and polyvinyl alcohol. 3,211,712, 1965.

106. McNeely, M. R.; Spute, M. K.; Tusneem, N. A.; Oliphant, A. R., Hydrophobic microfluidics. Proc. SPIE 1999, 3877, 210-220.

107. Meera Khan, P., Enzyme electrophoresis on cellulose acetate gel: zymogram patterns in mgh-mouse and man--Chinese hamster somatic cell hybrids. Arch Biochem Biophys 1971, $145,(2), 470-83$.

108. Mitrovski, S. M.; Elliott, L. C. C.; Nuzzo, R. G., Microfluidic devices for energy conversion: Planar integration and performance of a passive, fully immersed $\mathrm{H}_{2}-\mathrm{O}_{2}$ fuel cell. Langmuir 2004, 20, (17), 6974-6976.

109. Morra, M.; Occhiello, E.; Garbassi, F., Contact angle hysteresis in oxygen plasma treated poly (tetrafluoroethylene). Langmuir 1989, 5, (3), 872-876.

110. Nadzhafova, O. Y.; Zaitsev, V. N.; Drozdova, M. V.; Vaze, A.; Rusling, J. F., Heme proteins sequestered in silica sol-gels using surfactants feature direct electron transfer and peroxidase activity. Electrochem. Commun. 2004, 6, 205-209.

111. Nag, A.; Panda, B. R.; Chattopadhyay, A., Performing chemical reactions in virtual capillary of surface tension-confined microfluidic devices. Pramana Journal of Physics 2005, 65, (4).

112. Neumann, A. W.; Good, R. J.; Hope, C. J.; Sejpal, M., An equation-of-state approach to determine surface tensions of low-energy solids from contact angles. J. Colloid Interface Sci 1974, 49, (2), 291-304.

113. Nguyen, N. T.; Wu, Z., Micromixers - a review. J. Micromech. Microeng 2005, 15, (2), R1-R16.

114. Nieminen, J. A.; Abraham, D. B.; Karttunen, M.; Kaski, K., Molecular dynamics of a microscopic droplet on solid surface. Physical Review Letters 1992, 69, (1), 124-127.

115. Ogiwara, Y.; Koike, N.; Kubota, H.; Hata, Y., Factors affecting photografting of methacrylic acid on polyethylene film in liquid phase system. Journal of applied polymer science 1988, 35, (6), 1473-1481.

116. Oh, C. S. Microfluidic electrophoresis device. 5,904,824, 1999.

117. Olsson, E.; Kreiss, G., A conservative level set method for two phase flow. Journal of Computational Physics 2005, 210, (1), 225-246.

118. Osher, S.; Fedkiw, R. P., Level Set Methods and Dynamic Implicit Surfaces. Springer: 2002. 
119. Palecek, E.; Kizek, R.; Havran, L.; Billova, S.; Fojta, M., Electrochemical enzymelinked immunoassay in a DNA hybridization sensor. Analytica Chimica Acta 2002, 469, 7383.

120. Paynter, R. W., XPS studies of the ageing of plasma-treated polymer surfaces. Surface and Interface Analysis 2000, 29, (1), 56-64.

121. Phelan, R.; Weaire, D.; Brakke, K., Computation of equilibrium foam structures using the surface evolver. Experimental Mathematics 1995, 4, 181-192.

122. Pritchard, J. G., Poly (vinyl Alcohol): Basic Properties and Uses. Gordon and Breach: 1970.

123. Pugia, M. J.; Lott, J. A.; Profitt, J. A.; Cast, T. K., High-sensitivity dye binding assay for albumin in urine. Journal of Clinical Laboratory Analysis 1999, 13, (4), 180-187.

124. Ransohoff, J. A.; Woodard, D. G., Practical production of polyvinyl acetate emulsions by radiation catalysis. Proc. Jap. Conf. Radioisotop., 9th, Tokyo, 1969 1969, 598, (604).

125. Reyes, D. R.; Iossifidis, D.; Auroux, P. A.; Manz, A., Micro total analysis systems. 1. Introduction, theory, and technology. Anal. Chem 2002, 74, (12), 2623-2636.

126. Romero, L. A.; Yost, F. G., Flow in an open channel capillary. Journal of Fluid Mechanics 1996, 322, 109-129.

127. Rosario, R.; Gust, D.; Hayes, M.; Jahnke, F.; Springer, J.; Garcia, A. A., PhotonModulated Wettability Changes on Spiropyran-Coated Surfaces. Langmuir 2002, 18, (21), 8062-8069.

128. Rosario, R.; Gust, D.; Hayes, M.; Springer, J.; Garcia, A. A., Solvatochromic Study of the Microenvironment of Surface-Bound Spiropyrans. Langmuir 2003, 19, (21), 88018806.

129. Rossier, J. S.; Girault, H. H., Enzyme linked immunosorbent assay on a microchip with electrochemical detection. Lab on a Chip 2001, 1, (2), 153.

130. Rupcich, N.; Brennan, J. D., Coupled enzyme reaction microarrays based on pinprinting of sol-gel derived biomaterials. Analytica Chimica Acta 2003, 500, (1), 3-12.

131. Rye, R. R.; Mann, J. J. A.; Yost, F. G., The flow of liquids in surface grooves. Langmuir 1996, 12, (2), 555-565.

132. Rye, R. R.; Yost, F. G.; Mann Jr, J. A., Wetting Kinetics in Surface Capillary Grooves. Langmuir 1996, 12, (20), 4625-4627. 
133. Sankaran, R. M., Maskless etching of silicon using patterned microdischarges. Applied Physics Letters 2001, 79, (5), 593.

134. Sankaran, R. M., Hollow cathode sustained plasma microjets: Characterization and application to diamond deposition. Journal of Applied Physics 2002, 92, (5), 2406.

135. Sato, K.; Yamanaka, M.; Hagino, T.; Tokeshi, M.; Kimura, H.; Kitamori, T., Microchip-based enzyme-linked immunosorbent assay (microELISA) system with thermal lens detection. Lab on a Chip 2004, 4, (6), 570-575.

136. Schutze, A.; Jeong, J. Y.; Babayan, S. E.; Park, J.; Selwyn, G. S.; Hicks, R. F., The atmospheric-pressure plasma jet: a review and comparison to other plasma sources. IEEE Transactions on Plasma Science 1998, 26, (6), 1685-1694.

137. Seemann, R.; Brinkmann, M.; Kramer, E. J.; Lange, F. F.; Lipowsky, R., Wetting morphologies at microstructured surfaces. PNAS 2005, 102, (6), 1848-1852.

138. Senda, N.; Momotake, A.; Nishimura, Y.; Arai, T., Synthesis and Photochemical Properties of a New Water-Soluble Coumarin, Designed as a Chromophore for Highly Water-Soluble and Photolabile Protecting Group. Bulletin of the Chemical Society of Japan 2006, 79, (11), 1753-1757.

139. Seppala, M.; Rutanen, E. M.; Heikinheimo, M.; Jalanko, H.; Engvall, E., Detection of trophoblastic tumour activity by pregnancy-specific beta-1-glycoprotein. Int J Cancer 1978, $21,(3), 265-7$.

140. Sethian, J. A., Level set methods and fast marching methods. Cambridge University Press Cambridge: 1999.

141. Sipponen, P.; Ruoslahti, E.; Vuento, M.; Engvall, E.; Stenman, U., CEA and CEAlike activity in gastric cancer. Acta Hepatogastroenterol 1976, 23, (4), 276-9.

142. Squires, T. M.; Quake, S. R., Microfluidics: Fluid physics at the nanoliter scale. Reviews of Modern Physics 2005, 77, (3), 977-1026.

143. Stroock, A. D.; Dertinger, S. K. W.; Ajdari, A.; Mezic, I.; Stone, H. A.; Whitesides, G. M., Chaotic Mixer for Microchannels. Science 2002, 295, (5555), 647-651.

144. Stroock, A. D.; Whitesides, G. M., Controlling flows in microchannels with patterned surface charge and topography. Acc. Chem. Res 2003, 36, (8), 597-604.

145. Suk, J. W.; Cho, J. H., Capillary flow control using hydrophobic patterns. Journal of Micromechanics and Microengineering 2007, 17, N11-N15. 
146. Sullivan, D. E., Surface tension and contact angle of a liquid-solid interface. The Journal of Chemical Physics 1981, 74, 2604.

147. Sussman, M.; Fatemi, E.; Smereka, P.; Osher, S., An Improved Level Set Method for Incompressible Two-phase Flows. Computers \& Fluids 1998, 27, (5), 663-680.

148. Sussman, M.; Smereka, P.; Osher, S., A level set approach for computing solutions to incompressible two-phase flow. Journal of Computational Physics 1994, 114, (1), 146-159.

149. Swickrath, M. J., Mann, J. Adin, Wnek, Gary E., Surface-Directed Capillary Flow Systems. In Encyclopedia of Micro- and Nanofluidics, 1 ed.; Lee, D., Ed. Springer-Verlag: Heidelberg, In Press.

150. Tanner, W. B. Purification of polyvinyl acetate. 2,878,168, 1959.

151. Tolman, R. C., The Effect of Droplet Size on Surface Tension. Journal of Chemical Physics 2004, 17, 333.

152. Turkevich, L. A.; Mann, J. A., Pressure dependence of the interfacial tension between fluid phases. 1. Formalism and application to simple fluids. Langmuir 1990, 6, (2), 445-456.

153. Turkevich, L. A.; Mann, J. A., Pressure dependence of the interfacial tension between fluid phases. 2. Application to liquid-vapor interfaces and to interfaces of amphiphilic solutions. Langmuir 1990, 6, (2), 457-470.

154. Uotila, M.; Ruoslahti, E.; Engvall, E., Two-site sandwich enzyme immunoassay with monoclonal antibodies to human alpha-fetoprotein. J Immunol Methods 1981, 42, (1), 11-5.

155. Vestad, T.; Marr, D. W. M.; Oakey, J., Flow control for capillary-pumped microfluidic systems. Journal of Micromechanics and Microengineering 2004, 14, (11), 1503-1506.

156. Volkoviski, V., Sur les tourbillons en festons. CR Acad. Sci (Paris), Compt. Rend 1935, 200, 1285-1287.

157. Voller, A., Enzyme immunoassays with special reference to ELISA techniques. Journal of Clinical Pathology 1978, 31, (6), 507-520.

158. Voller, A.; Huldt, G.; Thors, C.; Engvall, E., New serological test for malaria antibodies. Br Med J 1975, 1, (5959), 659-61.

159. Wang, H.; Brown, H. R., Aliphatic Ketones as Photoinitiators for Photografting. Macromolecular Rapid Communications 2004, 25, (13), 1257-1262. 
160. Wang, H.; Brown, H. R.; Li, Z., Aliphatic ketones/water/alcohol as a new photoinitiating system for the photografting of methacrylic acid onto high-density polyethylene. Polymer 2007, 48, (4), 939-948.

161. Wang, H.; Iovenitti, P.; Harvey, E.; Masood, S., Numerical investigation of mixing in microchannels with patterned grooves. Journal of Micromechanics and Microengineering 2003, 13, (6), 801-808.

162. Washburn, E. W., The Dynamics of Capillary Flow. Physical Review 1921, 17, (3), 273-283.

163. Watanabe, M., Refreshable microfluidic channels constructed using an inkjet printer. Sensors and Actuators B: Chemical 2007, 122, (1), 141-147.

164. Watanabe, M., Refreshable microfluidic channels constructed using an inkjet printer. Sensors and actuators. B, Chemical 2007, 122, (1), 141-147.

165. Wenzel, R. N., Surface Roughness and Contact Angle. The Journal of Physical Chemistry 1949, 53, (9), 1466-1467.

166. West, J.; Michels, A.; Kittel, S.; Jacob, P.; Franzke, J., Microplasma writing for surface-directed millifluidics. Lab Chip 2007, 7, 981-983.

167. White, N. M.; Turner, J. D., Thick-film sensors: past, present and future. Meas. Sci. Technol 1997, 8, (1).

168. Wilkes, J. O., Microfluidics and Electrokinetic Flow Effects. In Fluid Mechanics for Chemical Engineers with Microfluidics and CFD, 2nd ed.; Prentice Hall: Upper Saddle River, 2006; pp 639-665.

169. Wilson, D. J.; Eccles, A. J.; Steele, T. A.; Williams, R. L.; Pond, R. C., Surface chemistry and wettability of plasma-treated PTFE. Surface and Interface Analysis 2000, 30, (1), 36-39.

170. $\mathrm{Wu}, \mathrm{T}$. C. Process for the production of poly(vinyl acetate) and poly(vinyl alcohol). 4,820,767, 1989.

171. Xia, Y.; Whitesides, G. M., Soft lithography. Angew. Chemie Int. Ed. 1998, 37, (5), 550-75.

172. Yang, L. J.; Yao, T. J.; Tai, Y. C., The marching velocity of the capillary meniscus in a microchannel. Journal of Micromechanics and Microengineering 2004, 14, (2), 220-225.

173. Young, T., Miscellaneous Works, Vol. by G. Peacock and J. Murray, London 1805, 418. 
174. Zhai, L.; Berg, M. C.; Cebeci, F.; Kim, Y.; Milwid, J. M.; Rubner, M. F.; Cohen, R. E., Patterned Superhydrophobic Surfaces: Toward a Synthetic Mimic of the Namib Desert Beetle. Nano Lett 2006, 6, (6), 1213-1217.

175. Zhang, C.; Xu, J.; Ma, W.; Zheng, W., PCR microfluidic devices for DNA amplification. Biotechnol. Adv 2006, 24, (3), 243-284.

176. Zhao, B.; Moore, J. S.; Beebe, D. J., Surface-Directed Liquid Flow Inside Microchannels. Science 2001, 291, (5506), 1023-1026.

177. Zhao, B.; Moore, J. S.; Beebe, D. J., Principles of surface-directed liquid flow in microfluidic channels. Anal. Chem 2002, 74, (16), 4259-4268.

178. Zhmud, B. V.; Tiberg, F.; Hallstensson, K., Dynamics of capillary rise. Journal of Colloid and Interface Science 2000, 228, (2), 263-269.

179. Zisman, W. A., Relation of the equilibrium contact angle to liquid and solid constitution. Adv. Chem. Ser 1964, 43, (1). 